\title{
Stellar Abundances in the Solar Neighborhood: The Hypatia Catalog
}

\author{
Natalie R. Hinkel ${ }^{1,2}$, F.X. Timmes ${ }^{1,3}$, Patrick A. Young ${ }^{1}$, Michael D. Pagano ${ }^{1}$, Margaret C. \\ Turnbull ${ }^{4}$
}

\begin{abstract}
We compile spectroscopic abundance data from 84 literature sources for 50 elements across 3058 stars in the solar neighborhood, within 150 pc of the Sun, to produce the Hypatia Catalog. We evaluate the variability of the spread in abundance measurements reported for the same star by different surveys. We also explore the likely association of the star within the Galactic disk, the corresponding observation and abundance determination methods for all catalogs in Hypatia, the influence of specific catalogs on the overall abundance trends, and the effect of normalizing all abundances to the same solar scale. The resulting large number of stellar abundance determinations in the Hypatia Catalog are analyzed only for thin-disk stars with observations that are consistent between literature sources. As a result of our large dataset, we find that the stars in the solar neighborhood may be reveal an asymmetric abundance distribution, such that a $[\mathrm{Fe} / \mathrm{H}]$-rich group near to the mid-plane is deficient in $\mathrm{Mg}$, Si, S, Ca, Sc II, Cr II, and Ni as compared to stars further from the plane. The Hypatia Catalog has a wide number of applications, including exoplanet hosts, thick and thin disk stars, or stars with different kinematic properties.
\end{abstract}

\section{Introduction}

One of the primary tools to understanding the history of the solar neighborhood, and more generally the Milky Way, is the chemical composition of stars. From the initial efforts of Russell (1929), Suess \& Urey (1956), and Bidelman (1960), to the more recent works of Anders \& Grevesse (1989), Edvardsson et al. (1993), Bensby et al. (2005), Valenti \& Fischer (2005), Asplund et al. (2009), and Lodders et al. (2009), compilations of stellar abundances provide an overall picture of the chemical evolution of the solar neighborhood. Notable results obtained over the past few decades include correlations of metallicity with age and Galactocentric distance, and whether the Sun is suitably "average" (Eggen et al. 1962; Twarog 1980; Feltzing et al. 2001; Robles et al. 2008a.b). Trends in the elemental abundances, and a limited number of isotopic abundances, relative to iron have also been observed over the whole metallicity range of the Galactic disk (Venn et al. 2004.

\footnotetext{
${ }^{1}$ School of Earth and Space Exploration, Arizona State University, Tempe, AZ 85287, USA

${ }^{2}$ San Francisco State University, Department of Physics and Astronomy, San Francisco, CA 94132, USA

${ }^{3}$ The Joint Institute for Nuclear Astrophysics, Notre Dame, IN 46556, USA

${ }^{4}$ Global Science Institute, P.O. Box 252, Antigo, WI 54409, USA
} 
Soubiran \& Girard 2005). For example, oxygen and the other $\alpha$-chain elements, relative to iron, vary systematically from being overabundant at $[\mathrm{Fe} / \mathrm{H}] \lesssim 1.0$ dex to roughly solar at $[\mathrm{Fe} / \mathrm{H}] \approx 0.0$. This decrease is widely taken to be caused by the contributions of supernovae Type Ia (SN Ia) in an average, well-mixed interstellar medium (Truran \& Cameron 1971; Tinsley 1980; Matteucci \& Greggio 1986; Lambert 1989; Wheeler et al. 1989; Timmes et al. 1995; Goswami \& Prantzos 2000: Gibson et al. 2003; Kobayashi et al. 2006; Krumholz et al. 2007; Prantzos 2008; Romano et al. 2010; Kobayashi \& Nakasato 2011).

Another tool to interpret the history of the solar neighborhood (taken throughout this paper to be stars within $150 \mathrm{pc}$ of the Sun) is the theory of stellar evolution, nucleosynthesis, and chemical evolution. By quantifying the ejecta from stars, this history can be reconstructed using theoretical models (Burbidge et al. 1957; Cameron 1957; Woosley \& Weaver 1995; Thielemann et al. 1996. Meynet \& Maeder 2002a; Siess et al. 2002; Ventura et al. 2002; Limongi \& Chieffi|2003; Karakas \& Lattanzio 2007; José \& Iliadis 2011). These models account for the initial mass function, star formation rate, stellar yields, inherited composition from the local interstellar medium (ISM), and are one of the keys to quantifying the formation of local solar neighborhood stars. Their results help provide important constraints on chemical evolution of the solar neighborhood, the Galactic disk, and other galaxies.

As the number of spectroscopic surveys of stars in the solar neighborhood increases, it has become tradition for authors of abundance surveys or chemical evolution models to compare their relative abundances to benchmark data sets for verification or validation. Typically, this involves comparing to Edvardsson et al. (1993), Reddy et al. (2003), Bensby et al. (2005), or Valenti \& Fischer (2005). However, the manner by which these comparisons are conducted varies drastically. Some authors provide statistical evaluations such as mean differences and standard deviations, some compare a few "typical" stars, and others graphically juxtapose entire catalogs. While there are certainly correlations between published data sets, there has been little discussion of the nuances, random uncertainties, and systematic biases of the compared data sets. It is, however, these idiosyncrasies that make interpreting trends between abundance catalogs challenging.

It is difficult and rare for one survey to systematically observe a large number of nearby stars and provide abundance determinations for a wide variety of elements. For example, Valenti \& Fischer (2005) reported the relative abundance in 1040 stars for only five elements, including iron. Alternatively, Reddy et al. (2003) analyzed spectra for 27 elements, but their study had only 181 stars. To achieve the most complete coverage of the solar neighborhood, the relative abundances from known literature sources must be combined. Such compilations have been undertaken by, for example, Venn et al. (2004) and Soubiran \& Girard (2005), with the amalgamation of thirteen and eleven published catalogs, respectively. Venn et al. (2004) contrasted the elemental abundance trends found in the nearby dwarf spheroidal galaxies with the overall trends found in the Milky Way in order to investigate whether, for example, the Milky Way halo could have been built from such systems. In a vein similar to our own, Soubiran \& Girard (2005) analyzed the abundance trends between $-1.3<[\mathrm{Fe} / \mathrm{H}]<+0.50$ for thin and thick disk stars. In both cases, the authors 
compiled detailed element abundances for a large number of stars in order to better understand overarching trends within the Milky Way.

The primary purpose of this paper is to present our Hypatia Catalog - so named after one of the first female astronomers who lived around 400 A.D.. The Hypatia Catalog (hereafter, Hypatia) is an unbiased compilation of spectroscopic abundance determinations from 84 literature sources for 50 elements across 3058 stars within 150 pc of the Sun. For the purposes of this paper, we show that the stellar abundances within Hypatia reflect the zeroth order trends consistent with published literature and mean galactic chemical evolution models. In \$2, we discuss the collation of the data, the inherent challenges in combining different data sources. In $\S 3$ we discuss the analysis of the stars in the Hypatia, whether they are thin- or thick-disk stars, and our attempts to mitigate some of the challenges in compiling such a large dataset. These adjustments to the data were only for the purposes of analysis and are not reflected in the published catalog. In $\$$, we describe more thoroughly how Hypatia was compiled. In $\$ 5$, we describe some of the details of the Hypatia Catalog as well as the abundance trends seen in Fe. In $\$ 6$, we present the abundance trends found in the $\alpha$-elements - C, O, Mg, Si, S, Ca \& Ti - in the solar neighborhood. In \$7, we describe the abundance trends for the odd-Z elements, namely Li, N, Na, Al, P, K \& Sc. The trends found in the iron-peak elements (V, Cr, Mn, Co \& Ni) are discussed in $\$ 8$ while those elements that are beyond the iron-peak $(\mathrm{Cu}, \mathrm{Zn}, \mathrm{Sr}, \mathrm{Y}, \mathrm{Zr}, \& \mathrm{Mo})$ are given in $\$ 9$. Finally, the neutron-capture elements $(\mathrm{Ru}$, $\mathrm{Ba}, \mathrm{La}, \mathrm{Ce}, \mathrm{Pr}, \mathrm{Nd}, \mathrm{Sm}, \mathrm{Eu}, \mathrm{Gd}, \mathrm{Dy}, \& \mathrm{~Pb}$ ) and their abundances are presented in $\$ 10$. Finally, in 11, we analyze an anomaly in the abundance measurements of a number of different elements that presented "gaps" in $[\mathrm{X} / \mathrm{Fe}]$ with for all $[\mathrm{Fe} / \mathrm{H}]$ and discuss the physical implications.

\section{Compilation of the Hypatia Catalog}

In this section we detail the scope of Hypatia and the manner in which datasets were chosen to be included. We also go into a more critical analysis of the individual works from which the abundance data were determined, given in a tabular format in Table 3.

\subsection{Abundance Datasets}

Numerous studies have analyzed the photospheres of stars in the solar neighborhood using photometric and spectroscopic techniques. Photometric investigations have treated a much larger number of stars relative to spectroscopic methods. However, photometric studies generally yield one global metallicity parameter, $[\mathrm{Fe} / \mathrm{H}]=\log \left(N_{F e} / N_{H}\right)_{*}-\log \left(N_{F e} / N_{H}\right)_{\odot}$, with units in dex, where $N_{F e}$ and $N_{H}$ are the number of iron and hydrogen atoms per unit volume, respectively. Despite the smaller number of stars analyzed with spectroscopy, the additional element abundances allow assessment of not just the overall metallicity, but the full chemical compositional range and evolution. Therefore, we have chosen to focus on published spectroscopic abundance catalogs. 
We compiled Hypatia with the spectroscopic abundance determinations of 50 element abundances for 3058 unique stars from published catalogs. Our exhaustive literature search considered all abundance determinations, of which we are aware, for main sequence F/G/K/M-type stars within 150 pc of the Sun. Table 1 shows a sample of the Hypatia Catalog which includes stellar $\mathrm{HIP} / \mathrm{HD} / \mathrm{BD}$ names, spectral type, distance, position, and the compiled abundances as given by each catalog, with reference. The complete catalog is given in the electronic version of this paper and the reduced catalog (per the discussion in $\$ 3$ and $\S$ s.cat) will be made available on Vizier. Efforts were made to include literature sources with abundance measurements for local stars published before the original submission of this (and any following) paper; any exclusion was not intentional. In addition, Hypatia has and will continue to be updated as newer and more precise abundances are determined. While these updates will be announced in subsequent papers, Hypatia will also be made available as an independent online database such that the community will have access to the most up-to-date data. Therefore, if a star within the solar neighborhood was measured for abundances other than iron, and it is $150 \mathrm{pc}$ of the Sun, it will be incorporated into Hypatia. The data sets that are contained in Hypatia are listed in Table 2, along with the number of stars meeting the above criteria and the element abundances determined therein. Throughout the paper, we give a more detailed description of each literature source and their method for determining stellar abundances.

A histogram of the number of stars measured for each element in Hypatia is shown in Fig. 1. All 3058 stars have a spectroscopically determined $[\mathrm{Fe} / \mathrm{H}]$. The next most frequently measured elements in Hypatia are Si (2320 stars), Ti (2237 stars), Ni (2225 stars), and O (2125 stars). There are only 33 stars in the solar neighborhood for which $[\mathrm{Ru} / \mathrm{H}]$ has been measured and only 20 stars for $[\mathrm{P} / \mathrm{H}]$. Fig. 1 also shows the relative paucity of stars in the solar neighborhood that have had their nitrogen, magnesium, and sulfur abundances determined, showing a clear direction for future studies of under-explored elements. This is primarily due to having too few absorption

lines, or lines that are too weak to separate from the continuum in the optical spectrum. The Hypatia Catalog also has abundance measurements for 13 singly ionized elemental species. While some catalogs measured only one ionization state when reporting an abundance determination, a number of catalogs combined the abundances from multiple ionization states. In Hypatia, an abundance of $[\mathrm{X} / \mathrm{Fe}]$ means that a catalog measured the neutral state, a combination of neutral and ionized state(s), or it was not specified. Whenever a catalog specifically mentioned it was only measuring the singly ionized state, we write $[\mathrm{X} I I / \mathrm{Fe}]$.

\subsection{Comparison of Datasets}

It was a large endeavor to compile and merge the 84 datasets in Hypatia, using services such as VIZIER, ADS, and SIMBAD as well as often times going to the original source in order to transcribe tables not available online. However, we recognize that it is naive to collect data without critically analyzing the individual works. Table 3 presents a comprehensive inventory of 


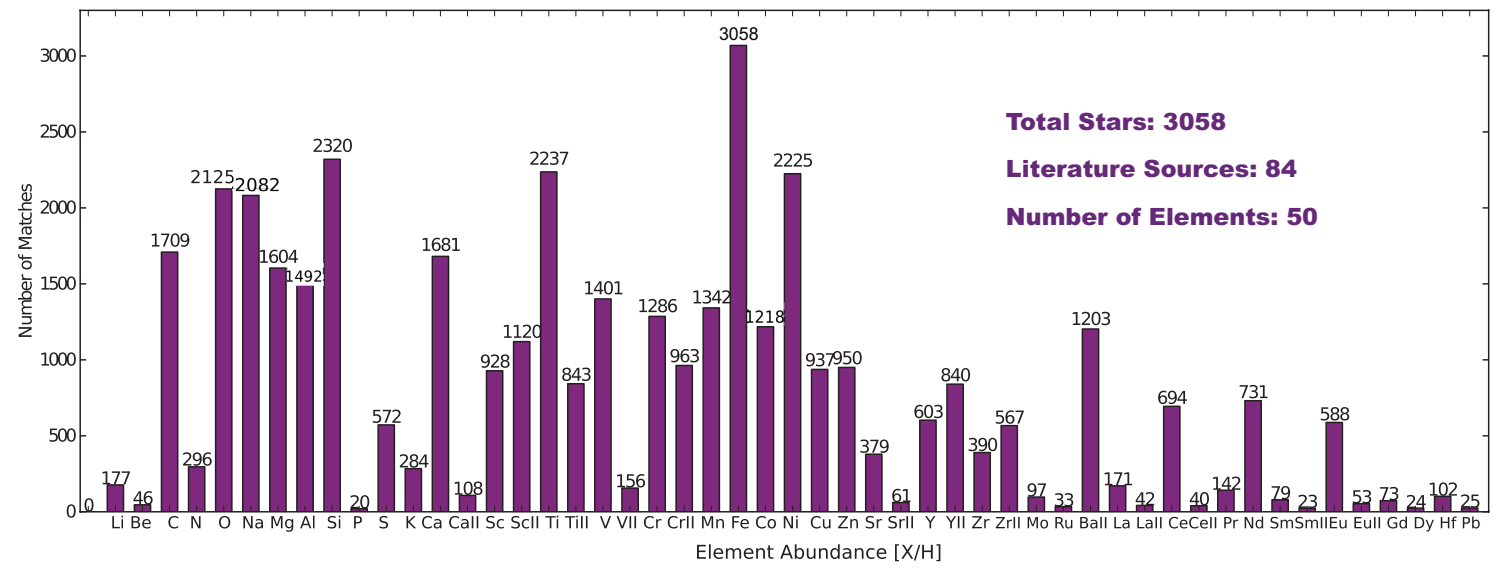

Fig. 1.- Number of stars in the Hypatia Catalog with measured abundances for 46 different element species.

the datasets with 20 or more stars found in Hypatia, including the telescopes and spectrographs, resolution, signal-to-noise $(\mathrm{S} / \mathrm{N})$, and wavelength range. We also documented the methods used by the individual authors to determine the stellar abundances, such as the stellar atmosphere models, equivalent width measurement techniques, whether they used iterative force-fits of observed and synthesized equivalent widths (i.e. curve-of-growth) or spectral fitting procedures, the solar abundances employed, and the number of Fe I and Fe II lines. Data were compiled with as much information as provided by the authors in their papers or subsequent references. Care was also taken to cite the appropriate sources, when available. Any error or exclusion was not intentional.

Looking at the catalogs given in Table 3 , it is clear that a wide variety of telescopes and spectrographs have been employed in order to determine stellar abundances in the local neighborhood. The telescope mirror sizes have a range from $1 \mathrm{~m}$ at the Cerro Tololo Inter-American Telescope (CTIO, Laird 1985) to the $10 \mathrm{~m}$ telescope at Keck using the High Resolution Echelle Spectrometer (HIRES, Boesgaard et al. 2011), where the average aperture size is $3.4 \mathrm{~m}$ for all of the telescopes listed in Table 3. A total of 18 of the 58 datasets combine spectra from two or more telescopes when determining their abundances. The resolution from the instruments varies from $\Delta \lambda / \lambda \approx$ 30000 (Gebran et al. 2010; Kang et al. 2011) to 120000 (Ramírez et al. 2007, 2009), where Gilli et al. (2006); Ramírez et al. (2007, 2009) cite a range in $\Delta \lambda / \lambda \approx 60000$ in their spectra alone, which was combined from multiple telescopes/spectrographs. Signal-to-noise was in general 100200 per spectral pixel, where the largest span (90-1350 per pixel) was encompassed by the spectra amalgamation in Ecuvillon et al. (2004, 2006).

The methods employed in order to determine the abundances from the spectra were also rather diverse. The preferred programs for determining stellar atmospheres were MARCS per Gustafsson et al. (1975) and ATLAS by Kurucz (1993). However, a number of alternative routines were also 
employed, such as EAGLNT (Edvardsson et al. 1993) and MAFAGS (Fuhrmann et al. 1997). Of the 58 datasets in Table 3 , a total of 48 of them determined equivalent widths of the absorption lines, for example using IRAF splot, ARES, or WIDTH9 (Kurucz 1993), and then used the curve-of-growth method, per MOOG (Sneden 1973) or Uppsala EQWIDTH, to calculate the majority of element abundances. Conversely, there were 10 datasets who used spectral fitting as their predominant method of determining stellar abundances. Many of the datasets in the Hypatia Catalog used Anders \& Grevesse (1989) and Grevesse \& Sauval (1998) for their solar abundance scale, however, many also calculated their own. As discussed in $\$ 3.2$, we have divided out the solar abundance used in each individual dataset and instead applied the abundance scale by Lodders et al. (2009).

Finally, while most of the catalogs that are incorporated into Hypatia published their line lists, not all of them measured the same element abundances, making a standardized comparison difficult. However, as mentioned in $\$ 2$, it was a requirement that any dataset added into Hypatia have $[\mathrm{Fe} / \mathrm{H}]$ abundance measurements. Therefore, we have included the number of Fe I and Fe II lines in the last column of Table 3. Through work as a part of the Stellar Stoichiometry: Workshop Without Walls held at Arizona State University during April 2013 (Hinkel et al. in prep), it was confirmed that the number of iron lines measured, below a certain threshold, drastically affects the determined $[\mathrm{Fe} / \mathrm{H}]$ abundance regardless of the abundance measurement technique employed. This is of particular interest given that the number of Fe I / Fe II lines varies from 20 / 1 (Jonsell et al. 2005) to 450 / 25 (Luck \& Heiter 2005) - ignoring those catalogs for which a definite count could not be made, namely Gustafsson et al. (1999) and Thévenin \& Idiart (1999).

From Table 3 we have found that a number of datasets included in the Hypatia Catalog use different telescopes/spectrographs at varying resolutions with a range in $\mathrm{S} / \mathrm{N}$, along with an assortment of prescriptions for modeling the stellar atmospheres and methods for measuring a variety of absorption lines in the spectra to determine abundances. However, we opted to avoid any sort of hierarchy on which to place the datasets, for fear that our "choices" may be inadvertently arbitrary or without the scientific rigor demonstrated in Lebzelter et al. (2012). We also found that scaling the abundances based on their stellar parameters was not viable per Ramírez et al. (2012). Instead, we examine the uniformity of the elemental abundances on a star-by-star basis between the datasets in 3.2 and exclude inconsistent measurements based on standard error in our further analysis.

\section{Analysis of Hypatia Stars}

Here we examine more closely the stars within the Hypatia Catalog, for example whether they are likely to be from the thin or thick disk population of the Galaxy. We also study the discrepancy in abundances when multiple datasets measure the same element within the same star. As a result of the following analysis, we are able to define a subset of abundances within the Hypatia Catalog that is both homogenous and robust. 


\subsection{Thin vs. Thick Disk Stars}

We separated the stars in the Hypatia Catalog into the Galactic components, for example thin-disk, thick-disk, or halo stars, based on their kinematics. Since chemical differences between the stellar populations are still debated, and chemical trends are the topic of our investigation, we decided that a conservative kinematic approach was the most appropriate. We used the updated and extended $U V W$ space velocities as determined for the Hipparcos Catalog by Anderson \& Francis (2012). The local standard of rest used in their calculations was $\left(U_{0}, V_{0}, W_{0}\right)=(-14.0,-14.5,-6.9)$ $\mathrm{km} \mathrm{s}^{-1}$ (Francis \& Anderson 2009). We followed the prescription in Bensby et al. (2003) in order to determine the probability that the Hypatia stars belonged to one of the Galactic components. By assuming the space velocities of the three stellar populations, as well as the number densities of the stellar components in the solar neighborhood, we were able to get a probability that a star belonged to a certain population. We assumed that $18 \%$ of the stars in the solar neighborhood originated in the thick disk, per Adibekyan et al. (2013). This local relative stellar density does not agree with Bensby et al. (2003, and references therein) who assumed a $6 \%$ thick-disk population. However, it is lower than the 25\% adopted in Mishenina et al. (2004) who later estimated an expected range of $2-15 \%$ with a larger sample size, similar to that found in Kordopatis et al. (2011), and larger than that noted in Reylé \& Robin (2001). Therefore, we found $18 \%$ to be a good median that was more conservative and agreed with the more recent studies. We did adopt the Gaussian distributions used in Bensby et al. (2003).

According to our probabilities, 2537 stars in Hypatia are thin-disk stars, 369 are thick-disk stars, 0 were from the halo, and 163 did not have space velocities according to Anderson \& Francis (2012). Therefore, we find that $12 \%$ of the Hypatia Catalog stars are likely from the thick-disk, as illustrated in red in the Toomre diagram (A. Toomre 1980 private communication with Sandage \& Fouts 1987) in Fig. 2(left). Our local stellar density calculations are consistent with the literature and lie within the expected range estimated by Mishenina et al. (2004). We did not include any of the probable thick-disk stars in our chemical abundance analysis in $\S \mathbf{5}-\S \mathbf{1 0}$.

A number of people have noted, for example Adibekyan et al. (2013); Bensby et al. (2003), and references therein, that the Galactic components of the solar neighborhood may be discerned via a combination of both kinematics and chemical abundances. In their paper, Adibekyan et al. (2013) described a population distinction occurring in an $[\alpha / \mathrm{Fe}]$ versus $[\mathrm{Fe} / \mathrm{H}]$ diagram, where " $\alpha$ " refers to the average of the $\mathrm{Mg}, \mathrm{Si}$, and $\mathrm{Ti}$ abundances. More pointedly, a region devoid of abundances measurements should occur approximately at $[\alpha / \mathrm{Fe}] \approx 0.2-0.3$ dex, with a possible "knee" at $[\mathrm{Fe} / \mathrm{H}]$ $\approx-0.3$ dex (see their Fig. 1). We have recreated the Adibekyan et al. (2013) figure in Fig. 2 (right) where the thin-disk stars are light yellow circles and the thick-disk stars are red triangles. The two populations in the Hypatia Catalog appear relatively well-mixed with respect to $[\alpha / \mathrm{Fe}]$, with few thick-disk stars that have lower $[\alpha / \mathrm{Fe}]$ and higher $[\mathrm{Fe} / \mathrm{H}]$. Our findings mimic the trends seen in Reddy et al. (2006); Pritzl et al. (2005); Tolstoy et al. (2003); Edvardsson et al. (1993), who also analyze the trends seen in $[\alpha / \mathrm{Fe}]$ compared to $[\mathrm{Fe} / \mathrm{H}]$ for thin- and thick-disk stars. They also do not show the same "knee" or empty region displayed in Adibekyan et al. (2013). 

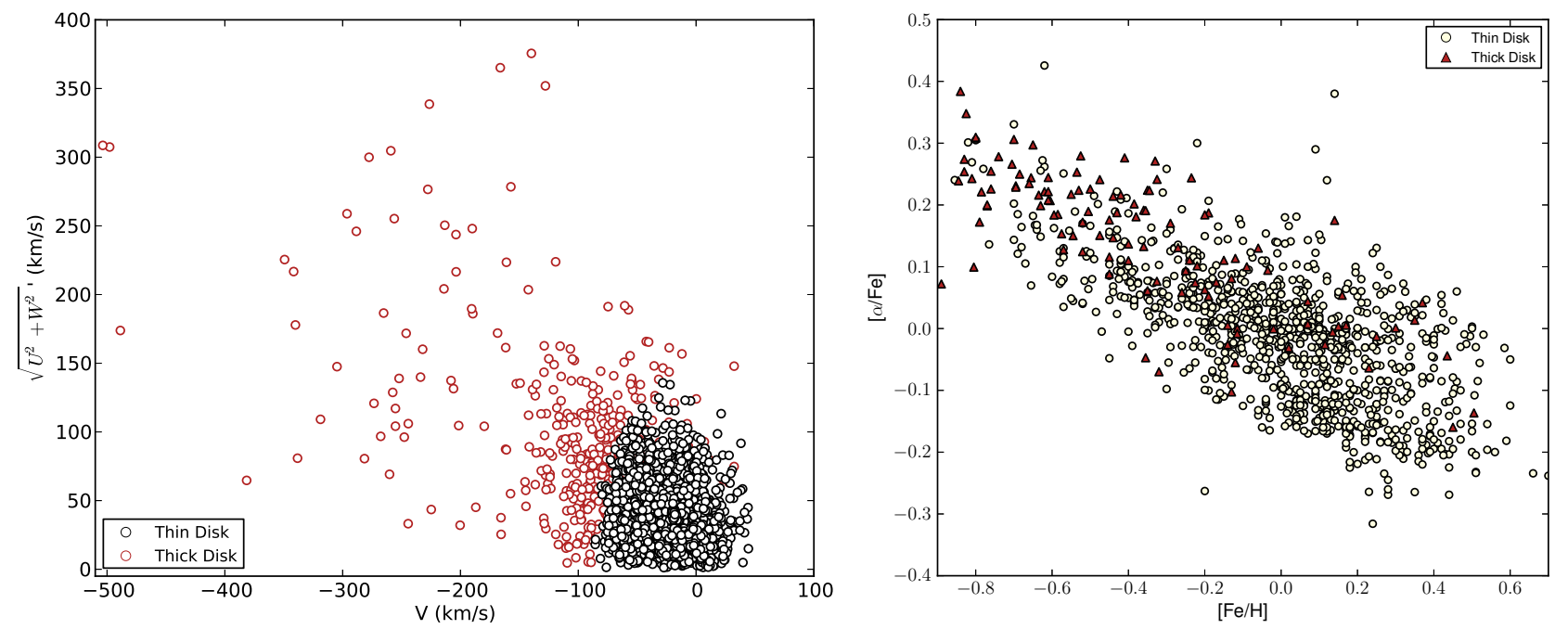

Fig. 2.- A Toomre diagram showing all of the stars in the Hypatia Catalog (left), where the stars most likely to be from the thick-disk are in red and those from the thin-disk are in black. To the right, a similarly color-coded plot recreating Figure 1 seen in Adibekyan et al. (2013), where they found the two populations separated by a gap in $[\alpha / \mathrm{Fe}]$, which was not seen using the Hypatia stars.

\subsection{Spread in the Elements}

Collecting abundance determinations from multiple authors over about a 25 year time span means at least the following differences between data sets: instrument zero points, resolution of the spectra, signal-to-noise ratios, oscillator strengths, line lists, equivalent widths, number of ionization stages used, LTE or non-LTE analysis, converged solar atmosphere models, curve-of-growth or spectral fitting, curve-of-growth program used, and adopted solar abundances. All of these factors may introduce systematic and stochastic differences between data sets. For example, Fig. 3 (top) shows the abundance measurements for six elements within five Hypatia stars. The circles are as labeled with the element name while all triangles designate $[\mathrm{Fe} / \mathrm{H}]$, each with respective error bars from the catalog from which it was measured. The variation between catalogs per element, the largest of which we call the spread, has a mean of 0.14 dex and a median of 0.11 dex for all elements in all stars in Hypatia. These values are on par if not larger than the error bars for most elements.

In addition to the abundances, stellar parameters may also suffer from inherent issues, depending on the reduction procedure. Torres et al. (2012) analyzed three methods for determining $\mathrm{T}_{\text {eff }}$, $\log (\mathrm{g})$, and $[\mathrm{Fe} / \mathrm{H}]$ in order to find the systematic differences between the techniques. They utilized both "constrained" spectroscopic determinations of the stellar parameters, by making use of the normalized semimajor axis $a / R_{*}$, as well as "unconstrained" determinations. Through these comparisons, they found that in the case of Stellar Parameter Classification (SPC) and Spectroscopy 
Made Easy (SME), both of which employ spectral synthesis and global $\chi^{2}$ minimization, the surface gravity was strongly correlated to the $\mathrm{T}_{\text {eff }}$ and $[\mathrm{Fe} / \mathrm{H}]$ measurements (see their Figures 2 and 4). In other words, when the $\log (\mathrm{g})$ values are larger, the $\mathrm{T}_{\text {eff }}$ and $[\mathrm{Fe} / \mathrm{H}]$ measurements increase due to the degeneracy between the stellar parameters. Additionally, they noted that the variation for each respective parameter between the "constrained" and "unconstrained" determinations increased as effective temperature increased (their Figure 6). Within the Hypatia Catalog, Fig. 3 shows the variation of $\mathrm{T}_{\text {eff }}$ and $\log (\mathrm{g})$ (middle and bottom, respectively) among many of the included datasets, determined for the abundances given at the top of the figure (when reported). For $\mathrm{T}_{\text {eff }}$, there is a spread of $\sim 200 \mathrm{~K}$, while $\log (\mathrm{g})$ can differs by a factor of $\sim 0.3$. The overall abundance determinations are sensitive to the adopted stellar values, especially $\log (\mathrm{g})$.

There is an accumulation of systematic and stochastic differences in the abundance measurements compiled to form Hypatia. Other authors have noted the difficulties in comparing different catalogs (e.g. Feltzing \& Gustafsson 1998; Bond et al. 2006), but only recently have they tried to overcome the challenges. Ramírez et al. (2012) analyzed the lithium abundances from nearby stars, supplemented by abundances from 7 other catalogs. They attempted to scale the abundances within the catalogs by taking into account the differing stellar parameters (namely, $\mathrm{T}_{\text {eff }}$ and $\log (g))$. However, the abundances did not vary with the stellar parameters in a consistent manner, not linearly or even as a standard function. Because stellar atmospheric models determine a wide variety of parameters, we agree with Ramírez et al. (2012) that attempting to correct for differing stellar parameters between catalogs will not be scientifically valuable. Therefore, we have decided to use a method similar to Roederer (2013). He combined 54 nearby stellar abundance catalogs and only used the highest quality abundances for repeat observations, making no attempt to correct for differing stellar parameters. His data was therefore unbiased towards one specific dataset, unlike Ramírez et al. (2012) who opted to normalize the other datasets to their own. Due to the range of possible issues, we have not included $\mathrm{T}_{\text {eff }}$ and $\log (\mathrm{g})$ for the stars in Hypatia.

We attempted to make the various catalogs in Hypatia more copacetic by putting all the different measurements on the same solar abundance scale, a correction that was also employed in Hinkel \& Kane (2013), see Table 3. Column 9. While some literature sources adjust their atomic data in order for their results to match certain solar values, this correction takes place before the stellar-to-solar comparison and therefore before the solar normalization, or re-normalization. Therefore, normalization to the same solar scale is the only correction available to us that helps make the data more comparable and does not involve recalculating the abundance determinations from every dataset. For example, per Table 1. Valenti \& Fischer (2005) reported HIP 400 to have $[\mathrm{Ti} / \mathrm{H}]=-0.28$ dex. In their paper, they cite Anders \& Grevesse (1989) as the source of their solar abundances, where $\log \epsilon(T i)=4.99$. To calculate the re-normalized abundances according to the solar measurements by Lodders et al. $(2009)$, where $\log \epsilon(\mathrm{Ti})=4.93$, then $[\mathrm{Ti} / \mathrm{H}]=-0.28+$ $4.99-4.93=-0.22$ dex. For all of the abundances within each star in the Hypatia Catalog, we found that the average difference between the abundance determination before and after the renormalization was $0.06 \mathrm{dex}$, with a median of $0.04 \mathrm{dex}$. These variations in abundance are larger 


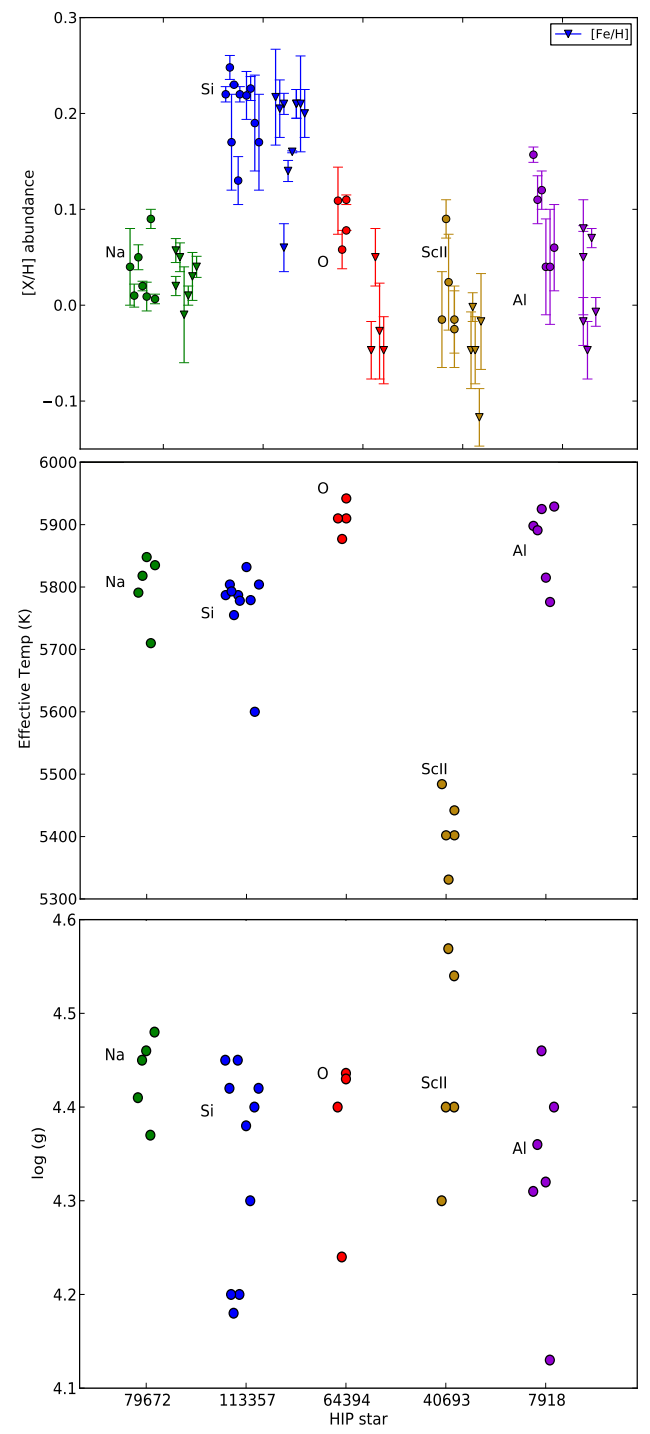

Fig. 3.- The representative spread (left) from different catalogs in the un-normalized abundance determinations (see text) for 6 element ratios for 5 stars, with quoted catalog errors such that the quoted uncertainty of individual measurements is less than the spread. The element ratios $[\mathrm{X} / \mathrm{H}]$ for a given star are denoted by circles and the corresponding $[\mathrm{Fe} / \mathrm{H}]$ abundances are shown as triangles. In the middle, the $\mathrm{T}_{\text {eff }}$ values for the same stars and $\log (\mathrm{g})$ are at the bottom. 
than the quoted error for many of the elements determined by the literature datasets. On the other hand, the average and median spread found in Hypatia only changed by 0.01 dex as a result of the re-normalization. In other words, the choice of solar abundance scale significantly affects the element abundance measurement, however, re-normalizing all of the datasets to the same solar abundance scale does not reduce the spread in the data. To be as consistent as possible, for all Hypatia calculations hereafter, we retain the element abundance values using the Lodders et al. (2009) solar abundance renormalization.

\section{The Structure of the Hypatia Catalog}

The Hypatia Catalog was compiled using PYTHON 2.7.3 with the following packages: MATPLOTLIB 1.3.1, NUMPY 1.8.0, and ATPY 0.9.6. Abundances tables (that measured both $[\mathrm{Fe} / \mathrm{H}]$ and at least one other element $[\mathrm{X} / \mathrm{Fe}]$ ) were either downloaded from VIZIER, converted to machine-

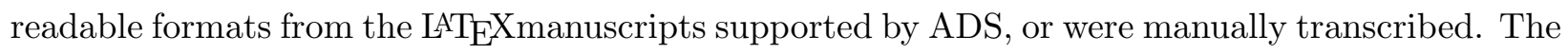
stellar names used in each individual table were then matched to the Hipparcos naming-scheme, which was chosen in order to provide continuity, incorporate as many stars as possible in the solar neighborhood, and provide a variety of stellar parameters (see Table 1). Datasets were only included in Hypatia if at least one star was within 150 pc of the Sun with a F, G, K, or M spectral type. In addition, we did not include abundances in our analysis that were determined using non-local thermodynamic equilibrium (NLTE) approximations.

If a dataset had stars that met the above criteria, than the element names, abundance values, and the literature source are recorded for each star, which is then incorporated into Hypatia. However, if a star within the dataset was found to likely originate from the thick disk or halo (per our discussion in 3.1 , that star was not incorporated in the compilation. Meaning, while a dataset may have been included, all of their stars are not necessarily part of the Hypatia Catalog. During this process, we also renormalized all of the stellar abundances, using Lodders et al. (2009) as

the standard. Once the renormalized abundances from all 84 datasets are compiled into Hypatia, then we are able to analyze the data. An element is first chosen to study, for example, sodium. Every thin disk star with a sodium abundance measurement is determined. In the cases where multiple datasets measured the same element abundance in the same star, so as not to favor any catalog like Roederer (2013), the median value for those measurements is used. However, we found that if the discrepancy between catalog measurements is too large, the median abundance value was unreliable. Rather than preferentially choose one catalog over another, we opted to eliminate those stars that do not have consistently measured element abundances from our analysis, in lieu of those that are more uniform and of higher quality. Therefore, any star with a spread in either $[\mathrm{X} / \mathrm{H}]$ or $[\mathrm{Fe} / \mathrm{H}]$ larger than the respective error bar was not included in the following (plotting) analysis. This cutoff value was used because it highlights those elements that are not well agreed upon by multiple literature sources. The total number of well-understood element abundances that remain for thin disk stars (see \$3.1) with spread per 
element less than error bar are listed in Table 5. Reduced abundances for these stars will be made available on Vizier.

In order to determine the representative error associated with each of the elements, we recorded the error as reported by each of the datasets from which that element was measured and averaged them together. Very few surveys determined star-by-star error values for the abundances, forcing us to also use a more general error bar per elements. In those cases where there were abundance errors per star, we took the average of all of the individual errors. While this method may mask some of the more precise abundance measurements with smaller associated errors, we found that it was better to take the more conservative approach by over-estimating the error, rather than under-estimating.

Going back to the example, for the case of sodium, there are 907 thin disk stars with one sodium abundance each that either came from 1) a single dataset or 2) the median value of multiple datasets measurements that have small variation between them. Because every star and every element abundance measurement are not created equal, this means that we are not plotting the same stars for every element. Instead, each element within each star is analyzed on a case-by-case basis in order to ensure that the quality of the data is high for our analysis, such that there were no compilation errors or idiosyncrasies. To create the $[\mathrm{X} / \mathrm{Fe}]$ vs. $[\mathrm{Fe} / \mathrm{H}]$ plots prevalent in $\$ 6.10$. we binned each of the stars according to distance from the Sun, as calculated from the RA, Dec, and parallax angles associated with the updated Hipparcos catalog (Anderson \& Francis 2012).

Quantifying systematic errors that could result from instrumental, atomic database, or stellar atmosphere models is beyond the scope of this paper, but we encourage the community to undertake such verification and validation studies. Therefore, we have decided to publish the Hypatia Catalog, see Table 1, containing only the original data by the literature authors, without any of our alteration or combination. In this way, others may use the compiled data to perform a more selective analysis as they see fit.

\section{Abundances in the Hypatia Catalog}

Combining 84 data sets that span about 25 years means there will be a spread among reported values for any element in many stars. We have have taken a many steps to address the issues to help make results generated with Hypatia meaningful and physical. We (a) exclude any probable thick-disk stars per the Bensby et al. (2003) method, illustrated in the Toomre diagram (Fig. 2 . left) in 3.1 (b) attempt to minimize the spread by renormalizing the abundances to a standard

solar abundance scale in $\$ 3.2$, (c) exclude stars with a large spread greater than the error bar per $\$ 4$. and (d) choose to use the median value of the spread avoid specific catalog bias when representing the abundance of a star in $\$ 4$. In this way, we only analyze thin-disk stellar abundances that are consistently measured between literature sources. The elemental abundances were then plotted in the traditional $[\mathrm{X} / \mathrm{Fe}]$ versus $[\mathrm{Fe} / \mathrm{H}]$ plane in Figs. 4430 . Representative error 
bars, compiled from the quoted observational uncertainties given by each literature source, for each element are placed in the upper right corners of each figure. We also show the overall trends in $[\mathrm{X} / \mathrm{Fe}]$ vs. $[\mathrm{Fe} / \mathrm{H}]$ by plotting $1 \sigma$ quantile regression.

We also analyze the data for trends in radial distance, z-height above the galactic disk, and directionality per the galactic center and anti-center. We have included a handful of these plots for a variety of elements to demonstrate any physical trends that may be present. There are also a small number of paired plots with abundances before and after the Lodders et al. (2009) renormalization. Literature sources that have contributed to the Hypatia Catalog are discussed throughout.

\subsection{Iron}

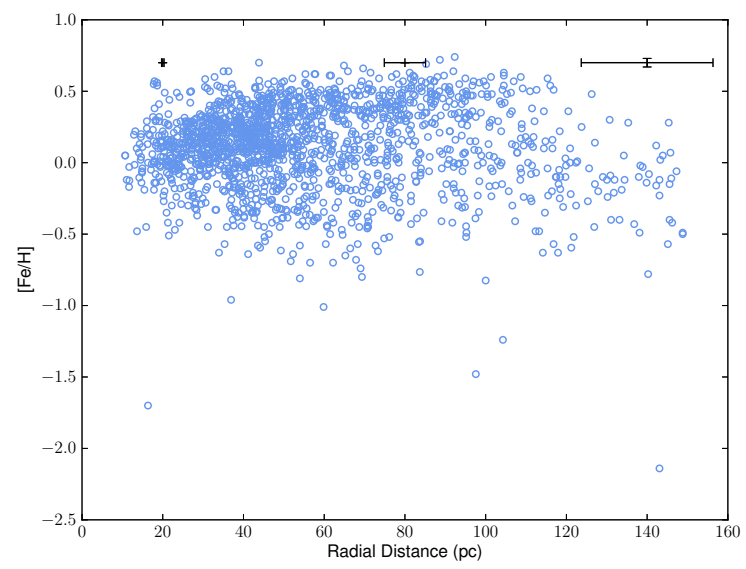

Fig. 4.- Median $[\mathrm{Fe} / \mathrm{H}]$ ratio for thin-disk, consistently measured stars in Hypatia as a function of radial distance from the Sun. Horizontal error bars along the top corresponding to the error in parallax angle used to calculate the distances.

The principle energy sources for most stars are hydrogen burning via the pp-chain or the CNO-cycle, or $\alpha$-chain burning. These energy sources underlie the processes through which most of the naturally occurring elements are created (Burbidge et al. 1957; Woosley \& Weaver 1995 , Thielemann et al. 2002; José \& Iliadis 2011). Iron is relatively easy to measure within stars due to the large number of absorption lines in the optical regime. Both SN Ia and core-collapse supernovae (SN II, SN Ib/c) produce iron, on different timescales as well as in different and debated amounts Chiappini et al. 1997; Thielemann et al. 2007; Prantzos 2008). And, due to the multiple production sites, the mean trend of iron increases monotonically in time within the ISM and thus it can act as a chronological indicator of nucleosynthesis (Wheeler et al. 1989; Pagel 1997; Vangioni et al. 2011). Therefore, as $[\mathrm{Fe} / \mathrm{H}]$ increases, so does the general timeline of chemical evolution - where we expect to see contributions from core-collapse supernovae for low values of $[\mathrm{Fe} / \mathrm{H}]$ and the effects 
from SN Ia at higher values (Wheeler et al. 1989; Matteucci \& Recchi 2001; Gibson et al. 2003 , Chiappini 2011). However, stars can migrate or scatter into or out of the solar neighborhood, and different galactic populations can have different star formation histories. In this case, $[\mathrm{Fe} / \mathrm{H}]$ does not necessarily represent the same timeline, which may introduce some ambiguity in using $[\mathrm{Fe} / \mathrm{H}]$ as a chronometer (Wielen et al. 1996; Gratton et al. 1996; Sellwood \& Binney 2002; Haywood 2008. Prantzos 2011).

Fig. 4 shows the median values of $[\mathrm{Fe} / \mathrm{H}]$ reported for 1713 stars in Hypatia, excluding those with a spread larger than the error (see $\$ 3.2$, with respect to the radial distance from the Sun. The horizontal error bars along the top are $0.32 \mathrm{pc}$ at $20 \mathrm{pc}, 5.1 \mathrm{pc}$ at $80 \mathrm{pc}$, and $16.3 \mathrm{pc}$ at 140 pc, showing how the fractional uncertainty in parallax angle affects the uncertainty in the distance calculation. Within our solar neighborhood's radius of $150 \mathrm{pc}$, there is a relatively constant scatter in $[\mathrm{Fe} / \mathrm{H}]$ at any distance, which may be due to a similar stellar origin or homogeneous mixture. The scatter in $[\mathrm{Fe} / \mathrm{H}]$ spans $\approx 1.5 \mathrm{dex}$, although the vast majority of the stars lie within $[-0.2$, 0.5] and are mostly within $80 \mathrm{pc}$ of the Sun. There are a few thin-disk stars, however, that are a relatively low in $[\mathrm{Fe} / \mathrm{H}]$, with abundances $<-1.0$ dex. Given that $150 \mathrm{pc}$ is small on galactic scales, near-solar values are to be expected.

\section{Chemical Abundances of $\alpha$-Elements: C, O, Mg, Si, S, Ca, \& Ti}

CNO nuclei are among the most abundant elements in the solar neighborhood Anders \& Grevesse 1989; Lodders et al. 2009; Asplund et al. 2009). They are important in stellar interiors as opacity sources (Iglesias \& Rogers 1996), as energy producers through the CNO cycle (Bethe

\& Critchfield 1938), and are essential building blocks of terrestrial biochemistry (Pace 2001). For consistent comparison, we have plotted all of the $\alpha$-elements using the same $\mathrm{x}$ - and $\mathrm{y}$-axis scales, such that $[\mathrm{Fe} / \mathrm{H}]=[-0.65,0.65]$ and $[\mathrm{X} / \mathrm{Fe}]=[-0.7,0.7]$, respectively.

\section{1. $\mathrm{C} \& \mathrm{O}$}

The element carbon, specifically the ${ }^{12} \mathrm{C}$ isotope, is formed via hydrostatic helium burning in stars, where the overall production is governed by the competition between the triple- $\alpha$ rate and destruction by the ${ }^{12} \mathrm{C}(\alpha, \gamma){ }^{16} \mathrm{O}$ rate (Iben 1991; Wallerstein et al. 1997; Busso et al. 1999 , Langanke et al. 2007, José \& Iliadis 2011; Bennett et al. 2012). Evolution of [C/Fe] as a function of $[\mathrm{Fe} / \mathrm{H}]$ for the 808 stars in the analysis of Hypatia is shown in Fig. 5 (left). A solar and relatively flat $[\mathrm{C} / \mathrm{Fe}]$ ratio is interesting because two competing sources come into play: intermediate and low mass stars begin depositing large amounts of carbon but no iron, while SN Ia start injecting significant amounts of iron but no carbon. To better understand the general trend of the data, the $\mathrm{x}$-axis has been divided into bins the size of the $[\mathrm{Fe} / \mathrm{H}]$ representative errorbar, or 0.05 dex. 

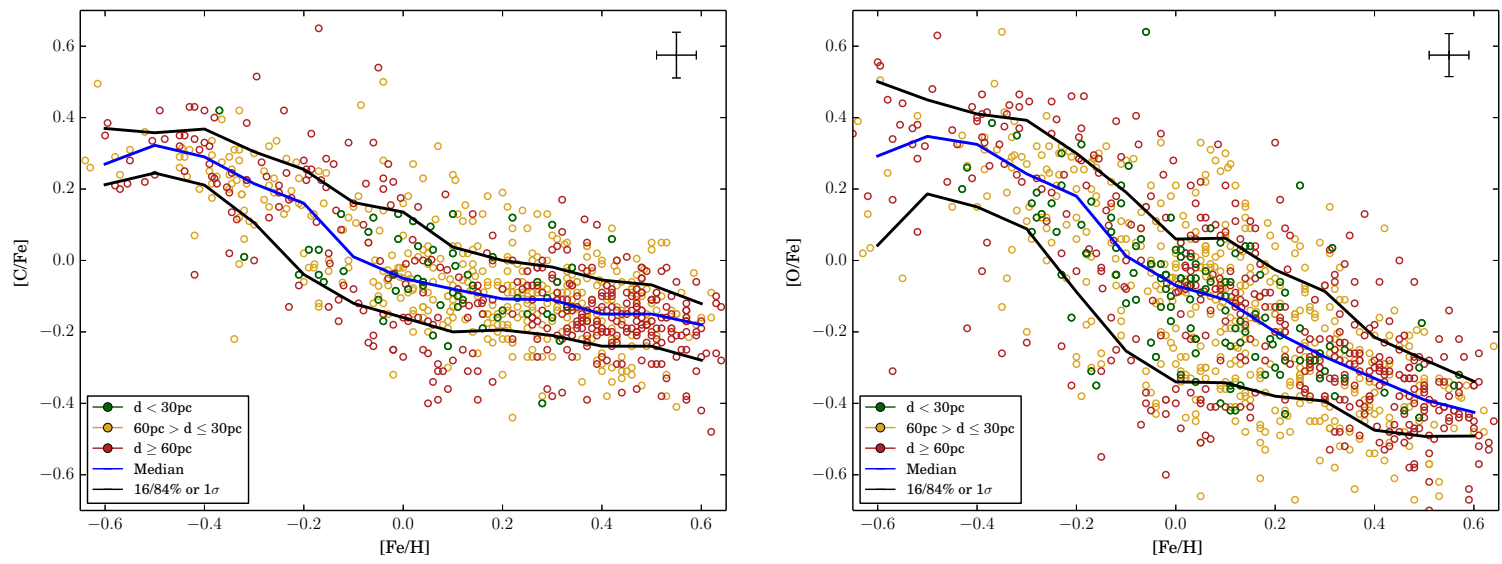

Fig. 5.- $[\mathrm{C} / \mathrm{Fe}]$ (left) and $[\mathrm{O} / \mathrm{Fe}]$ (right) ratio for stars in Hypatia as a function of $[\mathrm{Fe} / \mathrm{H}]$, with a representative observational error bar in the upper right. Each stellar abundance datapoint is colored according to the radial distance of the host-star. The median (blue) and $1 \sigma$ (between 16$84 \%$ ) quantile regression trends (black) are overlaid to better visualize the evolution of $[\mathrm{C} / \mathrm{Fe}]$ with increasing $[\mathrm{Fe} / \mathrm{H}]$. The median and percentile values at $[\mathrm{Fe} / \mathrm{H}]=-0.4,0.0$, and 0.4 dex are located in Table 4.

In each bin, the median (blue) was determined as well as the 16th and 84th percentile of the data (black) using quantile regression. In this way the majority of the data, or 1 standard deviation $(\sigma)$ pr $68 \%$ between the percentile trend lines, highlights how $[\mathrm{C} / \mathrm{Fe}]$ shifts with varying $[\mathrm{Fe} / \mathrm{H}]$. The blue and black curves in Fig. 5 (left) indicate a sharp decrease in $[\mathrm{C} / \mathrm{Fe}]$ for $[\mathrm{Fe} / \mathrm{H}]<0.0$ dex, followed by a shallower decline with increasing $[\mathrm{Fe} / \mathrm{H}]$. This suggests that $\mathrm{SN}$ Ia injected more iron than the intermediate and low mass stars injected carbon at an earlier epoch. There are $\sim 200$ giant-class stars in the Hypatia Catalog, many of which were from the Thevenin (1998) dataset and did not have carbon measurements. Only $\sim 50$ out of the total 808 stars with carbon abundances were giants, where 25 of those stars had $[\mathrm{C} / \mathrm{Fe}]<0.0$ dex. From Table 4 , we see that the 16 th and 84 th percentiles vary from the median by 0.11 and 0.16 dex respectively at $[\mathrm{Fe} / \mathrm{H}]=0.0$ dex. As $[\mathrm{Fe} / \mathrm{H}]$ increases to above-solar, where the majority of the data is located, the scatter of the $1 \sigma$ percentiles shrinks to 0.09 and 0.08 dex, respectively, at $[\mathrm{Fe} / \mathrm{H}]=0.4$. There also appears to be a slight concentration of stars with high $[\mathrm{Fe} / \mathrm{H}]$ but low $[\mathrm{C} / \mathrm{Fe}]$ content at distances greater than 60 $\mathrm{pc}$ - see the last paragraph in this section for further analysis with oxygen.

Laird (1985) determined carbon abundances in dwarf stars using intermediate resolution $(\Delta \lambda$ $=1 \AA)$ image tube spectra of the 3300-5250 $\AA$ band features of molecular CH. Many of these stars are in the analysis of the Hypatia Catalog, see \$4. Effective temperatures were found from calibrated R-I, $b-y$ and V-K color indices. Surface gravities were derived from the spectra and Strömgren photometry, supplemented with gravities based on parallax data and estimated masses. A differential analysis was adopted, and equivalent widths of the Fe I lines were used to determine 
the iron abundances. Since no individual $\mathrm{CH}$ lines could be detected in the spectra, LTE synthetic spectra determined the final abundances. An analysis of the $[\mathrm{C} / \mathrm{Fe}]$ ratio as a function of the effective temperature indicated a systematic offset, so a correction factor of 0.10 dex was applied to all the $[\mathrm{C} / \mathrm{Fe}]$ ratio. This correction factor was not used in the Hypatia Catalog, but it provides discouraging insight into the effect of variations between datasets, specifically for carbon.

Oxygen is a product of hydrostatic $\mathrm{He}, \mathrm{C}$, and $\mathrm{Ne}$ burning, with ${ }^{16} \mathrm{O}$ being the dominant isotope (Clayton 1968; Arnett 1996; Thielemann et al. 2002; Ekström et al. 2011). Three different oxygen features in the visible spectrum are used to determine oxygen abundances: the $\mathrm{O}$ I triplet at $7700 \AA$, the $[\mathrm{O} \mathrm{I}]$ doublet, or the $\mathrm{OH}$ lines. Oxygen abundances determined from the excitation feature $(9.15 \mathrm{eV}) \mathrm{O}$ I triplet at $7700 \AA$ are known to be sensitive to the temperature structure of the model atmosphere, as well as being affected by non-LTE corrections and convective inhomogeneities. Nevertheless, all 933 stars in the analysis of Hypatia for which $[\mathrm{O} / \mathrm{Fe}]$ was determined use the O I triplet. Most catalogs applied various empirical corrections by undertaking non-LTE calculations or providing an agreement with [O I] doublet determined abundances (e.g., Edvardsson et al. 1993 , Brugamyer et al. 2011).

Fig. 5 (right) shows $[\mathrm{O} / \mathrm{Fe}]$ versus $[\mathrm{Fe} / \mathrm{H}]$ for the stars in the analysis of Hypatia. The overall trend, shown by the blue and black trend lines, is classic $\alpha$-element, starting from core-collapse supernovae depositing large amounts of oxygen but no iron and later on SN Ia injecting significant amounts of iron but no oxygen (Gratton \& Ortolani 1986; Marcolini et al. 2009; Kobayashi \& Nakasato 2011). The $1 \sigma$ scatter in $[\mathrm{O} / \mathrm{Fe}]$ is at least $\sim 0.25$ dex for the entire range of $[\mathrm{Fe} / \mathrm{H}]$, increasing to $\sim 0.40$ dex ear the solar value of iron, or $[\mathrm{Fe} / \mathrm{H}]=0.0$ dex (see Table. 4). The inclusion of the Brewer \& Carney (2006) dataset had a direct impact on the expansion of the scatter in $[\mathrm{O} / \mathrm{Fe}]$ for near-solar values of $[\mathrm{Fe} / \mathrm{H}]$, although only by $\approx 0.1$ dex, which was on par with error.

A large number of stars with $[\mathrm{Fe} / \mathrm{H}]>0.2$ dex also have a radial distance greater than $60 \mathrm{pc}$ from the Sun, similar to the trend seen in the $[\mathrm{C} / \mathrm{Fe}]$ plot. We investigated this further and determined that the high $[\mathrm{Fe} / \mathrm{H}]$, low $[\mathrm{C} / \mathrm{Fe}]$ or $[\mathrm{O} / \mathrm{Fe}]$ abundances at large radial distances is due to the inclusion of Petigura \& Marcy (2011). Their dataset provided the largest incorporation of both carbon and oxygen abundances in 914 stars at an average distance of 52 pc (maximum 202 pc) from the Sun. Without their survey, stellar abundances exist in the high-iron, low-carbon/oxygen regime following the same trend, but in smaller numbers. While their data does not change the general features of the plots, it does smooth the progression for $[\mathrm{Fe} / \mathrm{H}]>0.2$ by adding more stars, specifically those at a distance beyond $60 \mathrm{pc}$. The preference for stars at high $[\mathrm{Fe} / \mathrm{H}]$ and large distances may be a result of 1 ) survey bias, since the majority of the stars measured by Petigura \& Marcy (2011) were at $[\mathrm{Fe} / \mathrm{H}]>0.0$ dex (their Fig. 14) or 2) physical inhomogeneities in the Galactic disk, such as those discussed in \$11, however more abundances at subsolar metallicities are needed before definite conclusions can be draw. The influence of the Petigura \& Marcy (2011) dataset is clear for the $[\mathrm{C} / \mathrm{Fe}]$ and $[\mathrm{O} / \mathrm{Fe}]$ abundances, but their data serves to solidify the trend observed by others. The addition of Ramírez et al. (2007) into Hypatia fulfilled a similar role for 
the $[\mathrm{O} / \mathrm{Fe}]$ abundances, in that their abundances covered much of $[\mathrm{Fe} / \mathrm{H}]<0.0$ dex, which suffered from small number statistics. Their data, too, did not drastically change the overall feature of the $[\mathrm{O} / \mathrm{Fe}]$ vs. $[\mathrm{Fe} / \mathrm{H}]$ trend, but allowed for more reliable statistics.

\section{2. $\mathrm{Mg}, \mathrm{Si}, \mathrm{S}$}

Magnesium is an $\alpha$-element whose dominant isotope ${ }^{24} \mathrm{Mg}$ is formed during hydrostatic carbon burning when a ${ }^{12} \mathrm{C}+{ }^{12} \mathrm{C}$ reaction creates the seed for ${ }^{23} \mathrm{Na}(p, \gamma){ }^{24} \mathrm{Mg}$, and during hydrostatic neon burning via ${ }^{20} \mathrm{Ne}(\alpha, \gamma){ }^{24} \mathrm{Mg}$ (Limongi \& Chieffi 2003; Karakas et al. 2006). Fig. 6 shows [Mg/Fe] as a function of $[\mathrm{Fe} / \mathrm{H}]$ after the renormalization to Lodders et al. (2009) (left) and according to the individual datasets (right). Similar to the other $\alpha$-elements, there is a general decrease in the $[\mathrm{Mg} / \mathrm{Fe}]$ abundance in Fig. 6 (left) as $[\mathrm{Fe} / \mathrm{H}]$ increases, due to the late injection of iron from SN Ia, and a flattening of $[\mathrm{Si} / \mathrm{Fe}]$ at super-solar metallicities (Matteucci \& Greggio 1986; Gibson et al. 2003; Romano et al. 2010; Kobayashi \& Nakasato 2011). However, the slope of [Mg/Fe] with [Fe/H] is shallower than the other two $\alpha$-elements already examined, carbon and oxygen. The average $1 \sigma$ scatter is $\sim 0.2$ dex in $[\mathrm{Mg} / \mathrm{Fe}]$, becoming slightly larger for $[\mathrm{Fe} / \mathrm{H}]=0.0$ dex and smaller at either extrema (see Table 4). This variation in $[\mathrm{Fe} / \mathrm{H}]$ supports the multiple productions sites predictions of Fenner et al. (2003).

In Fig. 5 (left), the stars at a further radial distance from the Sun had higher $[\mathrm{Fe} / \mathrm{H}]$ ratios but lower $[\mathrm{C} / \mathrm{Fe}]$; the opposite appears to be true with respect to $[\mathrm{Mg} / \mathrm{Fe}]$ such that stars at a distance of $60 \mathrm{pc}$ or less aren't as enriched in magnesium as iron. In comparison, the majority of stars at $60 \mathrm{pc}$ or more have both $[\mathrm{Mg} / \mathrm{Fe}]>0.0$ dex and $[\mathrm{Fe} / \mathrm{H}]<0.0$ dex. The majority of nearby stars exhibit super-solar $[\mathrm{Fe} / \mathrm{H}]$ abundance ratios. Overall, these trends hint at the possibility of there existing two "ensembles" of stars: one with high- $[\mathrm{Mg} / \mathrm{Fe}]$ but low $[\mathrm{Fe} / \mathrm{H}]$ at larger distances from the Sun and one with the opposite, separated by a "gap" around $[\mathrm{Mg} / \mathrm{Fe}] \approx-0.05$ dex. Edvardsson et al. (1993); Venn et al. (2004); Mishenina et al. (2008) all show [Mg/Fe] vs. [Fe/H] for disk-stars with a small "gap" separating the two groups that corroborate our trend. We compared the impact of the inclusion of many large datasets in Hypatia by temporarily excluding them and then reanalyzing the abundances. Specially, we temporarily omitted any catalog listed in Table 2 that had 200 stars or more by removing each dataset individually, recompiled the Hypatia Catalog, and then produced new plots. A comparison of the new plots-minus-one-catalog with respect to the full catalog plots gave a good indication on each dataset's overall influence. No catalogs caused a dramatic change in the $[\mathrm{Mg} / \mathrm{Fe}]$ trend which could explain the two "ensembles" or "gap." See \$11 for more discussion.

An obvious effect from the renormalization was a change in the $[\mathrm{Mg} / \mathrm{Fe}]$ vs. $[\mathrm{Fe} / \mathrm{H}]$ slope between the left and right plots in Fig. 6. The individual datasets used a variety of different solar abundances (see Table 3), such that $\log \epsilon(M g)$ had a range of 7.43-7.77. Since $\log \epsilon(M g)=7.54$ according to Lodders et al. (2009), which fell within the range of used of solar-Mg normalizations, the re-normalization affected each of the $[\mathrm{Mg} / \mathrm{Fe}]$ abundances differently. 
Looking at the $1 \sigma$ quantile regression lines between the two plots in Fig. 6, it is clear that the scatter is lower when using the solar abundances given by the individual datasets (see Table 4 for the mean and $1 \sigma$ values for the renormalized data). However, the absolute difference between the un-normalized $\left(A_{u n}\right)$ and re-normalized $\left(A_{r e}\right)$ abundances, or $\left|A_{u n}-A_{r e}\right|$, for all element ratios $([\mathrm{X} / \mathrm{Fe}])$ in the Hypatia Catalog has a mean of 0.06 dex and a median of 0.04 dex. In other words, to correct for varying solar abundance scales, the renormalization resulted in an average 0.06 dex shift in the abundance measurements. Specifically for Fig. 6, the mean and median absolute differanaences for $[\mathrm{Mg} / \mathrm{Fe}]=0.15$ and 0.12 dex, respectively, while the representative error is 0.07 dex. This variation is on par with representative error bar for most elements, especially those that are not neutron-capture, and has not been accounted for during survey comparisons. While the renormalization corrections create a drastic change in the data between the two plots in Fig. 6. these adjustments need to be adopted (amongst others) in order to uncover the physical trends in the data. Fig. 6 (right), without the solar renormalization, hides the two "ensembles" we noted to the left and discuss in $\$ 11$, and does not accurately reflect the scaling variations between datasets (see Table 3).
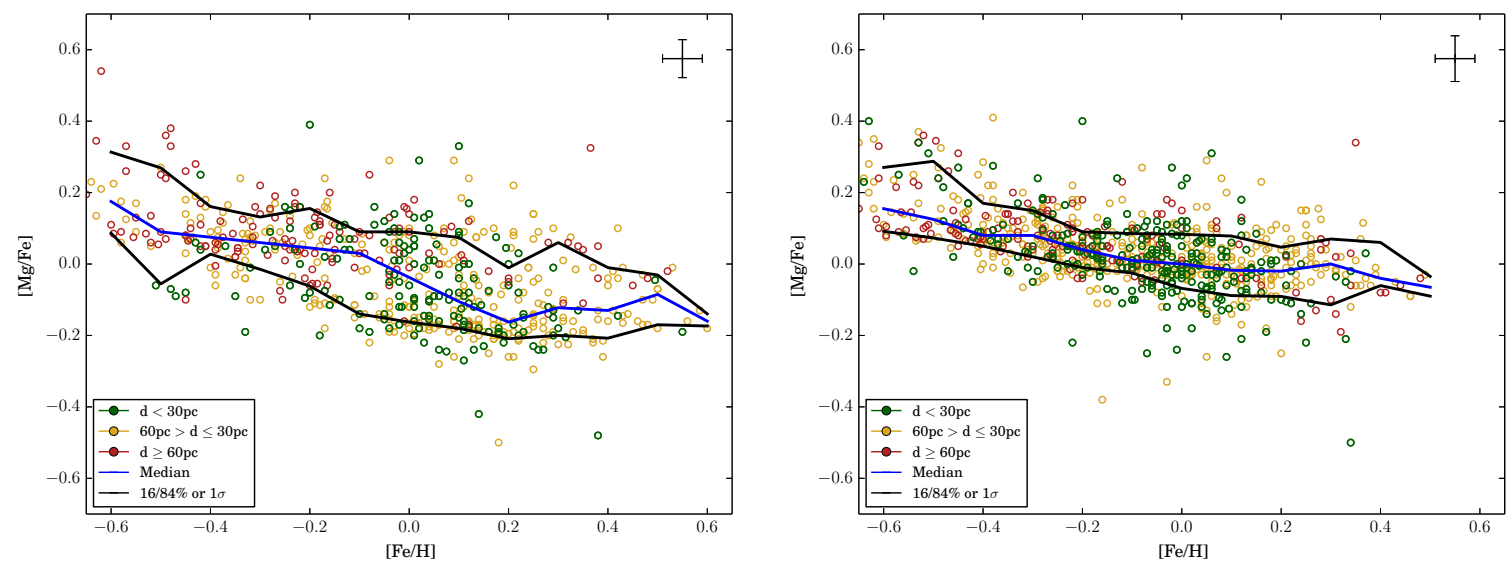

Fig. 6.- Similar to Fig. 5 but for magnesium, showing the data after being re-normalized (left) to Lodders et al. (2009) and as measured by the literature sources (right).

The dominant ${ }^{28} \mathrm{Si}$ isotope is produced by hydrostatic and explosive oxygen burning in massive stars (Arnett 1996). Fig. 7(left) shows silicon is a classic $\alpha$-chain element such that [Si/Fe] decreases with increased $[\mathrm{Fe} / \mathrm{H}]$. The $[\mathrm{Si} / \mathrm{Fe}]$ ratio has the most entries in the Hypatia Catalog, being measured for 1098 thin-disk stars with well agreed upon literature determinations. There is a $1 \sigma$ scatter $\approx$ 0.18 dex about the median trend (black line) at any given $[\mathrm{Fe} / \mathrm{H}]$, which is at a maximum around solar, 0.23 dex (see Table 4). Similar to [C/Fe] in Fig. 5(left) and [Mg/Fe] in Fig. 6 (left), there is a slight gap in abundances for $[\mathrm{Si} / \mathrm{Fe}]$ around $\approx-0.1$ dex. However, this "gap" is sub-solar as opposed to $[\mathrm{C} / \mathrm{Fe}]$ and the second, lower "ensemble" of stars with higher $[\mathrm{Fe} / \mathrm{H}]$ seem to be comprised of stars that have a radial distance of $60 \mathrm{pc}$ or less, similar to $[\mathrm{Mg} / \mathrm{Fe}]$ in Fig. 6 (left). For all of the 
stars in the lower "ensemble," about half of them $(\sim 290)$ originated from the Valenti \& Fischer (2005) catalog. The remaining $\sim 240$ stars were contributed by other datasets, such a Fulbright (2000); Gilli et al. (2006); Takeda (2007); Mishenina et al. (2008); Neves et al. (2009); Delgado Mena et al. (2010). As a test regarding the influence of the Valenti \& Fischer (2005) dataset, we recompiled the Hypatia Catalog without the inclusion of their measurements and found that the "gap" was still present at $[\mathrm{Si} / \mathrm{Fe}] \approx-0.1$ dex for all $[\mathrm{Fe} / \mathrm{H}]$. Rather than cover up the "gap," the inclusion of Valenti \& Fischer (2005) reinforced the trend delineated by the other datasets, seen in Fig. 5 (left).

The differences between these two "ensembles" indicate that there may be abundance correlations with distance and that the local neighborhood is not homogeneously mixed (see $\$ 11$ for more discussion).
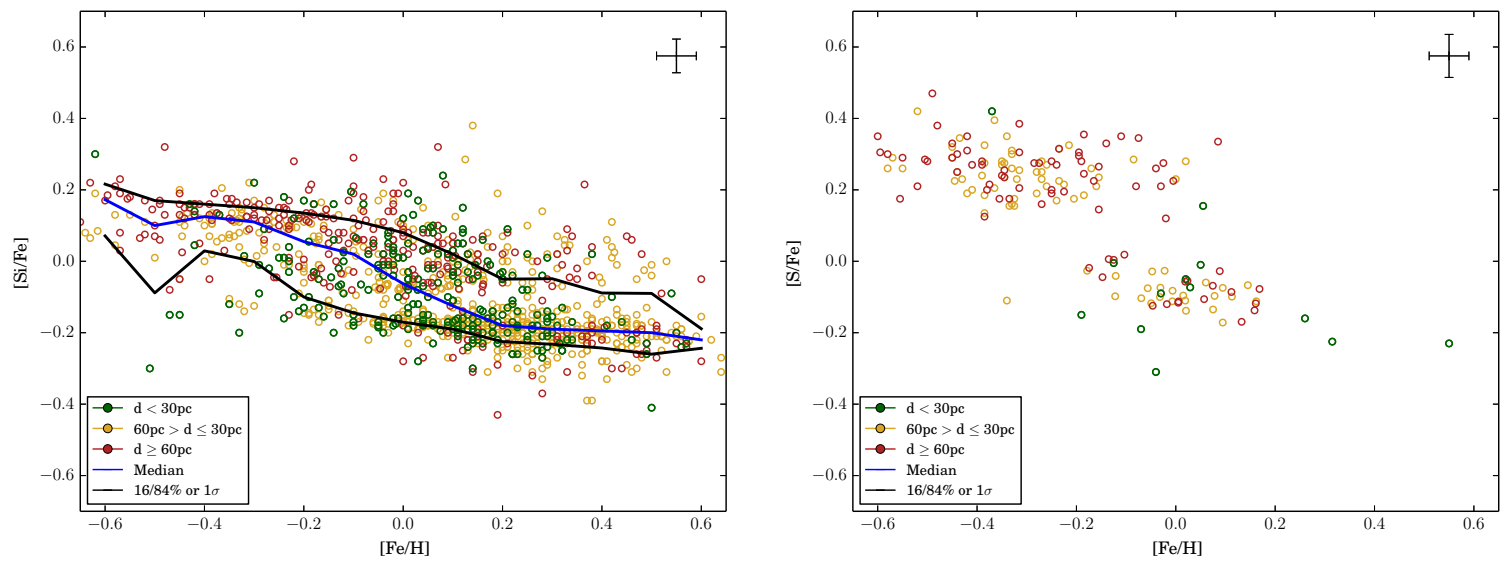

Fig. 7. - Same as Fig. 5 but for silicon (left) and sulfur (right). [Si/Fe] is one of the most common measurements in the analysis of Hypatia (1098 stars), with less entries for [S/Fe] (162 stars). Due to small number statistics, the median and percentile trend lines could not be accurately determined for [S/Fe], which has less than 250 stellar measurements (see Table 5).

Sulfur is produced within massive stars, via hydrostatic and explosive oxygen and burning (Clayton 1968; Heger et al. 2000; Rauscher et al.|2002; Limongi \& Chieffi|2003). There are relatively fewer stars, 162 in the analysis of Hypatia, for which sulfur has been measured, as shown in Fig. 7 (right). This is due to absorption lines being too weak in the visible spectrum or blended to separate from the continuum, making it difficult to determine an accurate abundance (Francois 1987). Takeda et al. (2005) reports significant, $\approx 0.2$ dex, non-LTE corrections affecting several lines used in the determination of $\mathrm{S}$ and $\mathrm{Zn}$ abundances in $\mathrm{F}, \mathrm{G}$, and $\mathrm{K}$ stars. The small number of stars in Fig. 7 (right) made the calculation of the median and $16 / 84 \%$ percentile regression lines impossible. Like other $\alpha$-elements, there is decrease in $[\mathrm{S} / \mathrm{Fe}]$ as $[\mathrm{Fe} / \mathrm{H}]$ increases from $\approx-0.6$ dex to $\approx 0.6$ dex and a slope similar to $[\mathrm{Si} / \mathrm{Fe}]$ (left). As previously noted for $[\mathrm{Si} / \mathrm{Fe}]$ vs. $[\mathrm{Fe} / \mathrm{H}],[\mathrm{S} / \mathrm{Fe}]$ exhibits a gap in element abundance between [0.0, 0.1] dex (see 11 for more discussion). The 
scatter in $[\mathrm{S} / \mathrm{Fe}]$ is $\approx 0.3$ dex over the entire $[\mathrm{Fe} / \mathrm{H}]$ range shown, but the two "ensembles" makes it hard to estimate. Mishenina et al. (2008) also show two ensembles when analyzing the [Si/Fe] vs. $[\mathrm{Fe} / \mathrm{H}]$ abundance trends.

Luck \& Heiter (2005) reported the abundances for $\mathrm{Mg}$, Si, and $\mathrm{S}$, as well as for 25 other elements, in $114 \mathrm{~F}, \mathrm{G}, \mathrm{K}, \mathrm{M}$ stars within 15 pc of the Sun. Table 2 shows 110 of these stars are in the Hypatia Catalog. Their high signal-to-noise spectra (in excess of $\approx 150$ per spectral pixel) were taken between 1997 and 2003 using the Sandiford Cassegrain Eschelle Spectrograph attached to the $2.1 \mathrm{~m}$ telescope at McDonald Observatory. They determined the solar flux spectrum by using differential analysis, with Callisto as the reflector. The model atmospheres were determined by MARCS75 (Gustafsson et al. 1975). Photometry was acquired through the General Catalogue of Photometric Data (Hauck \& Berthet 1991). Surface gravities $\log g$ values and Fe abundances were obtained by iterating until the $[\mathrm{Fe} / \mathrm{H}]$ value from both $\mathrm{Fe}$ I and Fe II were equal. Overall abundance uncertainties for $[\mathrm{X} / \mathrm{Fe}]$ were determined on a per element basis.

\section{3. $\quad \mathrm{Ca} \& \mathrm{Ti}$}

Calcium is an $\alpha$-element whose dominant, double magic isotope ${ }^{40} \mathrm{Ca}$ is produced by oxygen burning in massive stars (Woosley \& Weaver 1995). While most of the catalogs within the analysis of Hypatia determined their calcium abundances through Ca I, two catalogs (Allende Prieto et al. 2004, Gebran et al. 2010) used Ca II lines, shown in Fig. 8 (right). Allende Prieto et al. (2004) compared the derived abundances from the neutral and ionized lines, and reported the abundance for Ca I and II differed by 0.25 dex due to the broadening of the wings in the line profiles. These dissimilarities could be mollified by a change in the surface gravity and $\mathrm{T}_{e f f}$, at the expense of weakening the Ca I line. Therefore, their final abundances were derived from the Ca II $8662 \AA$ line, since this was less blended than the Ca II $8498 \AA$ line.

Fig. 8 shows how $[\mathrm{Ca} / \mathrm{Fe}]$ exhibits the same trend with $[\mathrm{Fe} / \mathrm{H}]$ as other $\alpha$-elements. However, the shallow slope over the $[\mathrm{Fe} / \mathrm{H}]$ range suggests that calcium production by massive stars is more closely balanced by iron production from SN Ia. There is $\approx 0.09-0.19$ dex in the $1 \sigma$ scatter for $[\mathrm{Ca} / \mathrm{Fe}]$ as $[\mathrm{Fe} / \mathrm{H}]$ increases from -0.4 dex to 0.4 dex, per Table 4 . The abundances for $[\mathrm{Ca}$ II/Fe] vs. $[\mathrm{Fe} / \mathrm{H}]$ are not shown because the total number of stars, after potential thick-disk stars and abundance measurements beyond error bar were removed, totaled only 8 stars.

It also appears that stars that are further away from the Sun, at a distance greater than 60 pc (red circles), exhibit generally higher abundances of $[\mathrm{Ca} / \mathrm{Fe}]$. However, a closer investigation reveals that the majority of stars with $[\mathrm{Ca} / \mathrm{Fe}]<-0.1$ dex were measured solely by Neves et al. (2009). While they did not place a direct distance cut on the stars they analyzed, their data originates from the HARPS GTO (see Table 3), which do not observe stars that are greater than 56 pc from the Sun. The abundances from Neves et al. (2009) provided [Ca/Fe] measurements from $\approx 400$ unique (i.e. only measured by them) stars. Their methodology is consistent with others in 
the field (see Table 3) such that their own comparison with literature sources did not show any significant differences. Without the inclusion of the Neves et al. (2009) survey, there are a number of stars in the region with high $[\mathrm{Ca} / \mathrm{Fe}]$ and low $[\mathrm{Fe} / \mathrm{H}]$, as measured by other surveys. A few of these stars are located at distances $>60 \mathrm{pc}$ from the Sun. As a result, the feature that separates the large-distance, high $[\mathrm{Ca} / \mathrm{Fe}]$ and low $[\mathrm{Fe} / \mathrm{H}]$ stars from their near-distance, low $[\mathrm{Ca} / \mathrm{Fe}]$ and high $[\mathrm{Fe} / \mathrm{H}]$ counterparts may not be physical. To be certain, we would either need to obtain more abundance measurements for stars a distances $>60 \mathrm{pc}$ from the Sun to obtain better statistics or reanalyze all of the stellar data from the surveys in the Hypatia Catalog with Ca I lines using a standardized method.

In a similar vein, we found that a number of stars that had a solar value of $[\mathrm{Ca} / \mathrm{Fe}] \mathrm{but}$ with $[\mathrm{Fe} / \mathrm{H}]>0.2$ dex originated from Trevisan et al. (2011). Further study found that Trevisan et al. (2011) contributed a unique and small outcrop of stars at high $[\mathrm{Fe} / \mathrm{H}]$ but near solar-value of $[\mathrm{X} / \mathrm{Fe}]$ for three elements: $\mathrm{Ca}, \mathrm{Ti}$, and Ni. Their Figure 21 confirms these variations with respect to other catalogs, namely Bensby et al. (2003, 2004); Mishenina et al. (2004, 2008); Reddy et al. (2003, 2006). They also report an average difference in abundance determinations within $\sim 0.2$ dex compared to Neves et al. (2009); Valenti \& Fischer (2005), which is much greater than standard error for $\mathrm{Ca}, \mathrm{Ti}$, and $\mathrm{Ni}$. This is unlike other cases where features in the abundance distribution may be dominated by a single survey, but are still present with reduced numbers of stars when the survey is removed, like the "gaps" in abundances (see $\$ 11$.

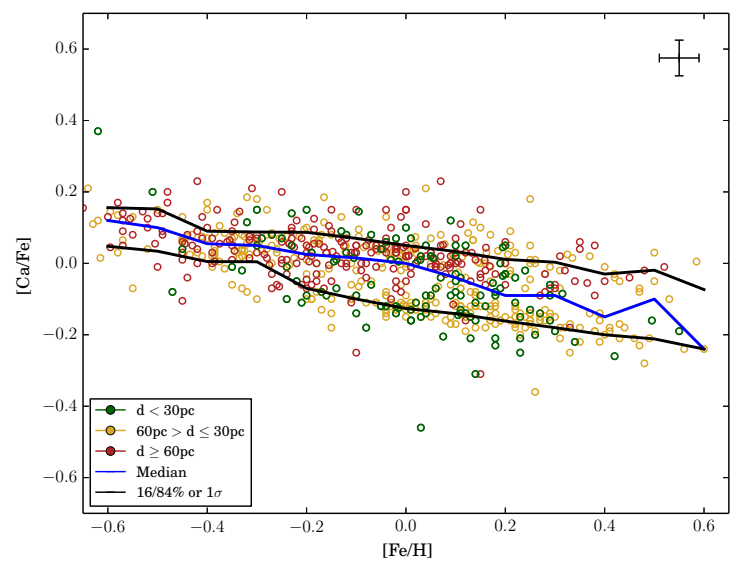

Fig. 8.- Same as Fig. 5 but for calcium.

Titanium is produced in massive stars by explosive burning processes in core-collapse supernovae (Woosley et al. 1973; Arnett 1996, Limongi \& Chieffi 2003). Like calcium, titanium abundances have been determined with two ionization states as shown in Fig. 9. Since there are a large number of spectral lines for Ti I and Ti II in the optical spectrum, titanium is one the more commonly measured elements in Hypatia (see Fig. 1). While a number of catalogs measured abundances using both the Ti I and Ti II lines (Bond et al. 2008; Gratton et al. 2003; Neves et al. 

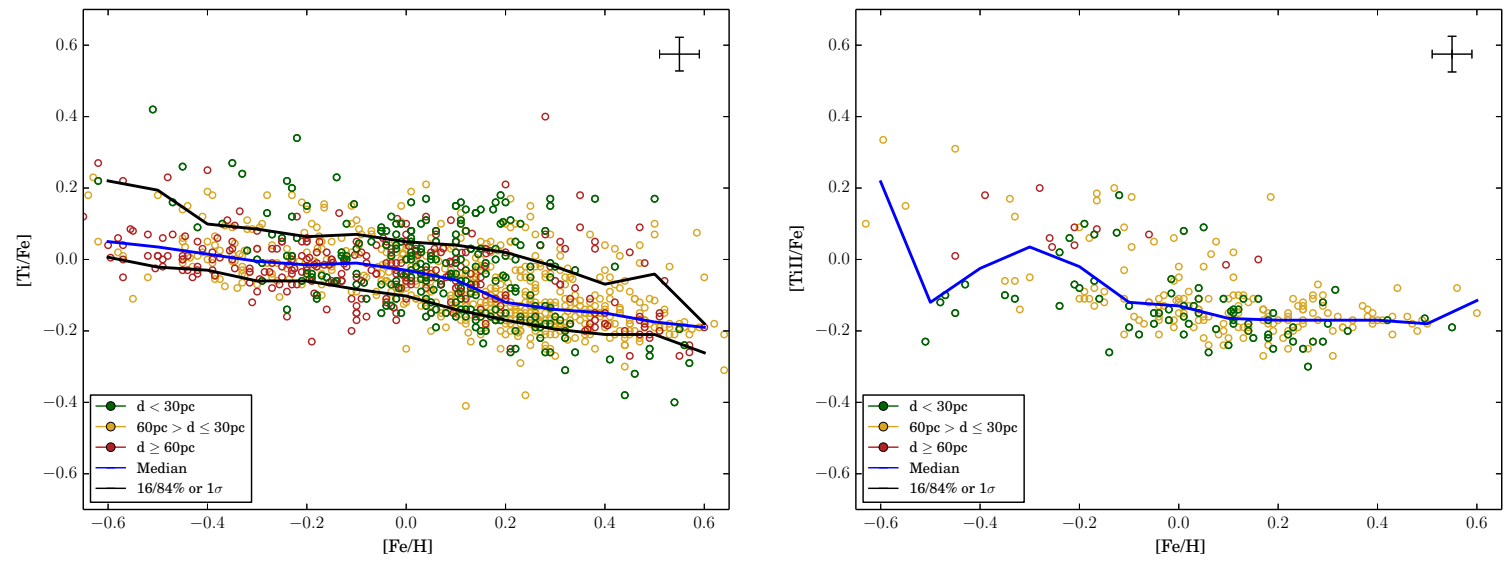

Fig. 9. - Same as Fig. 5 but for neutral (left) and ionized (right) titanium. Due to small number statistics, the percentile trend lines could not be accurately determined for [TiII/Fe], which has less than 250 stellar measurements (see Table 5).

2009; Takeda et al. 2007), Bergemann (2011) found that abundances can vary by 0.1 dex or larger when comparing pure Ti I and Ti II line determinations. Most catalogs, though, used the Ti I lines alone.

The $[\mathrm{Ti} / \mathrm{Fe}]$ binned trend with $[\mathrm{Fe} / \mathrm{H}]$, shown by the trend lines in Fig. 9 (left), suggests an evolution similar to the other $\alpha$-elements. Although, it should be noted that the dominant isotope is produced by alpha chain nucleosynthesis, being a beta decay product of the alpha isotope ${ }^{48} \mathrm{Cr}$. There is $\mathrm{a} \approx 0.15$ dex $1 \sigma$ scatter in $[\mathrm{Ti} / \mathrm{Fe}]$ over the entire range of $[\mathrm{Fe} / \mathrm{H}]$ in Fig. 9 (left). For $[\mathrm{Fe} / \mathrm{H}]<0.0$ dex, stars at larger distances (red) tend to show near-solar $[\mathrm{Ti} / \mathrm{Fe}]$, while stars at smaller distances tend to exhibit lower $[\mathrm{Ti} / \mathrm{Fe}]$ and higher $[\mathrm{Fe} / \mathrm{H}]$. The Valenti \& Fischer (2005) catalog contributed the majority of stars with $[\mathrm{Fe} / \mathrm{H}]>0.1$ dex, or $\sim 80 \%$ of their stars with titanium abundances. Out of those with enriched iron, only $8 \%$ were at a distance greater than 60 pc from the Sun, where the average was 36 pc. The distance-abundance trend seen for $[\mathrm{Ti} / \mathrm{Fe}]$ may therefore be a product of target selection and is left for further investigation. However, the steady decline of $[\mathrm{Ti} / \mathrm{Fe}]$ with increasing $[\mathrm{Fe} / \mathrm{H}]$ was confirmed by other surveys who measured stars in this region, but with much a much smaller sample of stars. Similarly, Trevisan et al. (2011) contributed the majority of stars with $[\mathrm{Fe} / \mathrm{H}]>0.2$ dex and $[\mathrm{Ti} / \mathrm{Fe}]>0.0$ dex, as previously discussed, but was not the lone source for stars in this region.

The median trend for [Ti II/Fe] is similar to [Ti/Fe], Fig. 9 (right), however with fewer stars such that the $1 \sigma$ trend lines cannot be accurately determined. The median trend indicates that the slope of $[\mathrm{TiII} / \mathrm{Fe}]$ becomes more shallow as $[\mathrm{Fe} / \mathrm{H}]$ increases, which may be a result of smallernumber statistics. There are significantly fewer stars with [Ti II/Fe] determinations at distances greater than 60 pc. Both Venn et al. (2004); Mishenina et al. (2008) saw separate ensembles in 
their abundance determinations for $[\mathrm{Ti} / \mathrm{Fe}]$. However, possibly due to the separation of neutral and ionized titanium in our analysis, we do not reproduce their results.
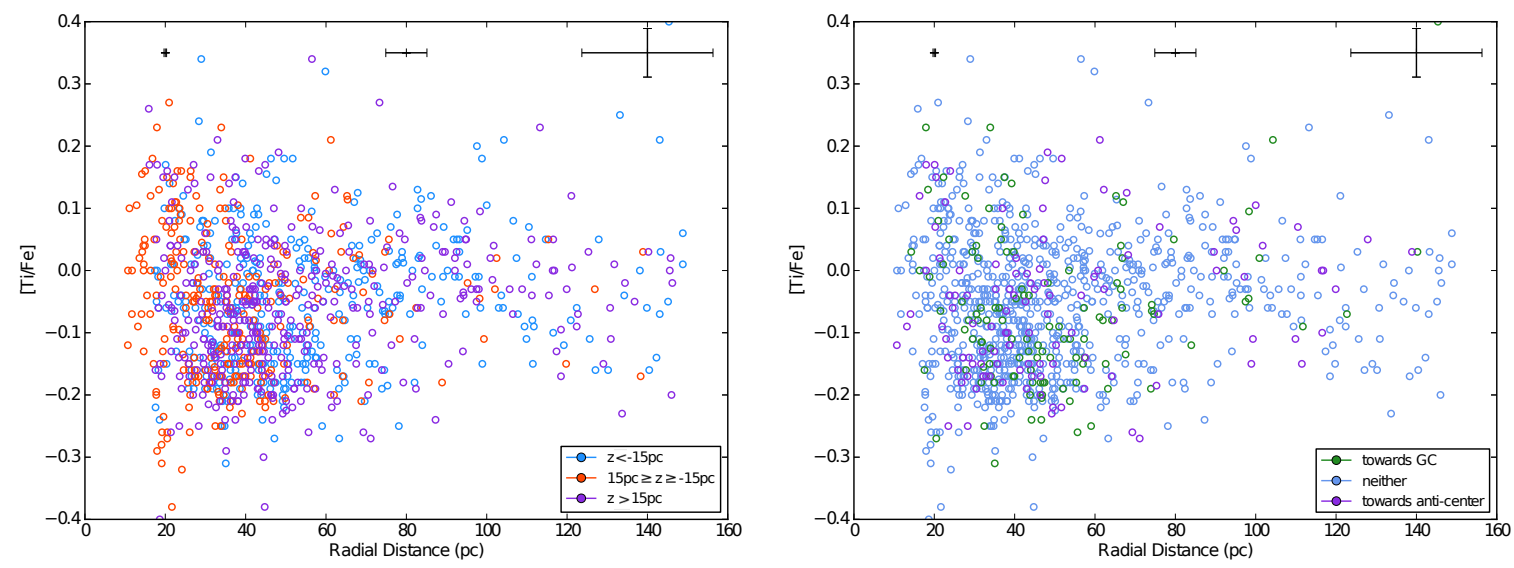

Fig. 10. - The same $[\mathrm{Ti} / \mathrm{Fe}]$ abundances as given in Fig. 9 but with respect to distance, where the abundance trends are shown with respect to height above the Galactic plane (left) and direction to the Galactic center and anti-center (right). The error bars are similar to Fig. 4.

Fig. 10 (left) shows [Ti/Fe], an $\alpha$-element, as a function of distance with error bars similar to Fig. 4. On the left, the stars are colored according to their height above the Galactic plane, z. The $[\mathrm{Ti} / \mathrm{Fe}]$ abundances show little dependence on height above the plane, where they appear evenly mixed at all radial distances, although fewer stars near the mid-plane were measured at further distances (red circles). And the majority of stars in the analysis of Hypatia that have [Ti/Fe] abundance measurements are located within 60 pc of the Sun. Fig. 10 (right) shows those same stars, but colored according to their position toward the galactic center. Here as well, there was no discernible trend with distance, although fewer stars located towards the Galactic center were measured at further radial distances.

Fig. 11 shows $[\mathrm{Na} / \mathrm{Ca}]$ (left) and $[\mathrm{Si} / \mathrm{Ca}]$ (right) as a function of $[\mathrm{Fe} / \mathrm{H}]$. The $[\mathrm{Na} / \mathrm{Ca}]$ evolution, an odd-Z element to an $\alpha$-element ratio, shows a change in slope of $[\mathrm{Na} / \mathrm{Ca}]$ at $[\mathrm{Fe} / \mathrm{H}] \approx 0.0$ dex (Marcolini et al. 2009). Production of sodium and calcium were roughly equivalent at smaller $[\mathrm{Fe} / \mathrm{H}]$, but sodium dominates calcium as $[\mathrm{Fe} / \mathrm{H}]$ increases, with a relatively constant $\approx 0.2 \operatorname{dex} 1 \sigma$ scatter in $[\mathrm{Na} / \mathrm{Ca}]$ for all $[\mathrm{Fe} / \mathrm{H}]$. This trend may be due to SN Ia or intermediate- to low-mass stars injecting additional sodium relative to calcium at later times. The outlying stars with super-solar $[\mathrm{Na} / \mathrm{Ca}]$ and $[\mathrm{Fe} / \mathrm{H}]>-0.2$ may be due to late contributions form asymptotic giant branch (AGB) stars. In contrast, $[\mathrm{Si} / \mathrm{Ca}]$ with $[\mathrm{Fe} / \mathrm{H}]$ (Fig. 11, right) shows a flat and solar trend within error. The $\approx 0.2-0.1$ dex $1 \sigma$ scatter shows how silicon and calcium, both $\alpha$-elements, are dominated by contributions from massive stars which co-produce both Si and Ca. The similar nucleosynthetic origin sites, along with the relative ease of measuring all three elements ( $\mathrm{Na}$, Si, and $\mathrm{Ca}$ ) within stars (see Fig. 1), may explain the small scatter for these abundance ratios. Other implied subtleties 

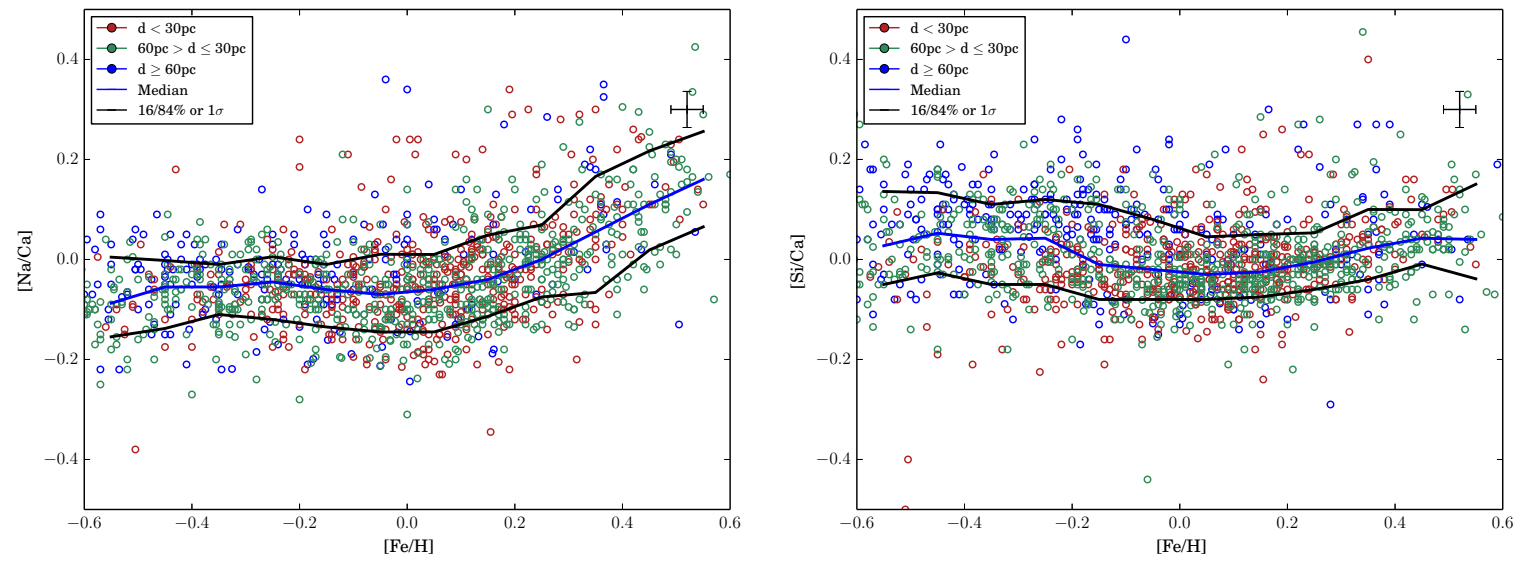

Fig. 11. - Similar to Fig. 5 but for $[\mathrm{Na} / \mathrm{Ca}]$ (left) and [Si/Ca] (right) as a function of $[\mathrm{Fe} / \mathrm{H}]$. may warrant future consideration.

\section{Odd-Z Elements (N, Na, Al, K, \& Sc)}

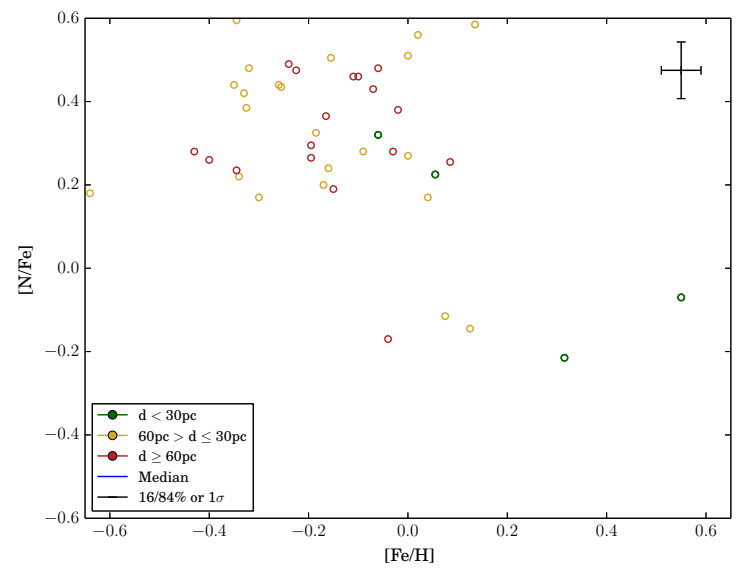

Fig. 12. $-[\mathrm{N} / \mathrm{Fe}]$ as a function of $[\mathrm{Fe} / \mathrm{H}]$ with the same format as Fig. 5. Due to small number statistics, the median and percentile trend lines could not be accurately determined for $[\mathrm{N} / \mathrm{Fe}]$, which has less than 250 stellar measurements (see Table 5).

Isotopes of nitrogen are produced in stars by the CNO cycle Arnett 1996, where primary nitrogen is usually produced as a convective helium burning shell mixes into a hydrogen shell, where $\mathrm{C}$ and $\mathrm{O}$ nuclei form nitrogen with nearly explosive consequences (Talbot \& Arnett 1974 . Meynet \& Maeder 2002a; Ekström et al. 2008; Karakas 2010). Fig. 12 shows [N/Fe] with respect 
to $[\mathrm{Fe} / \mathrm{H}]$, where the axes for all the odd-Z elements are now $[\mathrm{Fe} / \mathrm{H}]=[-0.65,0.65]$ and $[\mathrm{X} / \mathrm{Fe}]=$ $[-0.6,0.6]$. There are $\sim 15$ times fewer stars for which $[\mathrm{N} / \mathrm{Fe}]$ has been measured as compared to the $[\mathrm{C} / \mathrm{Fe}]$ ratio in our analysis of the Hypatia Catalog (see $\$ 4$ and Table 5 , making it a priority measurement for future observations. As a result, neither the median nor the $1 \sigma$ regression lines could be determined. The majority of $[\mathrm{N} / \mathrm{Fe}]$ abundances are above solar for $[\mathrm{Fe} / \mathrm{H}]<0.2$ dex, with few measurements for stars with $[\mathrm{Fe} / \mathrm{H}]$ above solar. The scatter in $[\mathrm{N} / \mathrm{Fe}]$ is rather significant for all $[\mathrm{Fe} / \mathrm{H}]$ abundances. If physical, the large scatter suggests ${ }^{14} \mathrm{~N}$ was produced as a primary element, since $[\mathrm{N} / \mathrm{Fe}]$ is relatively constant with $[\mathrm{Fe} / \mathrm{H}]$ (Laird 1985; Carbon et al. 1987). However, given the difficulty in measuring $[\mathrm{N} / \mathrm{Fe}]$, the large scatter may be due to discrepancies between data reduction techniques.
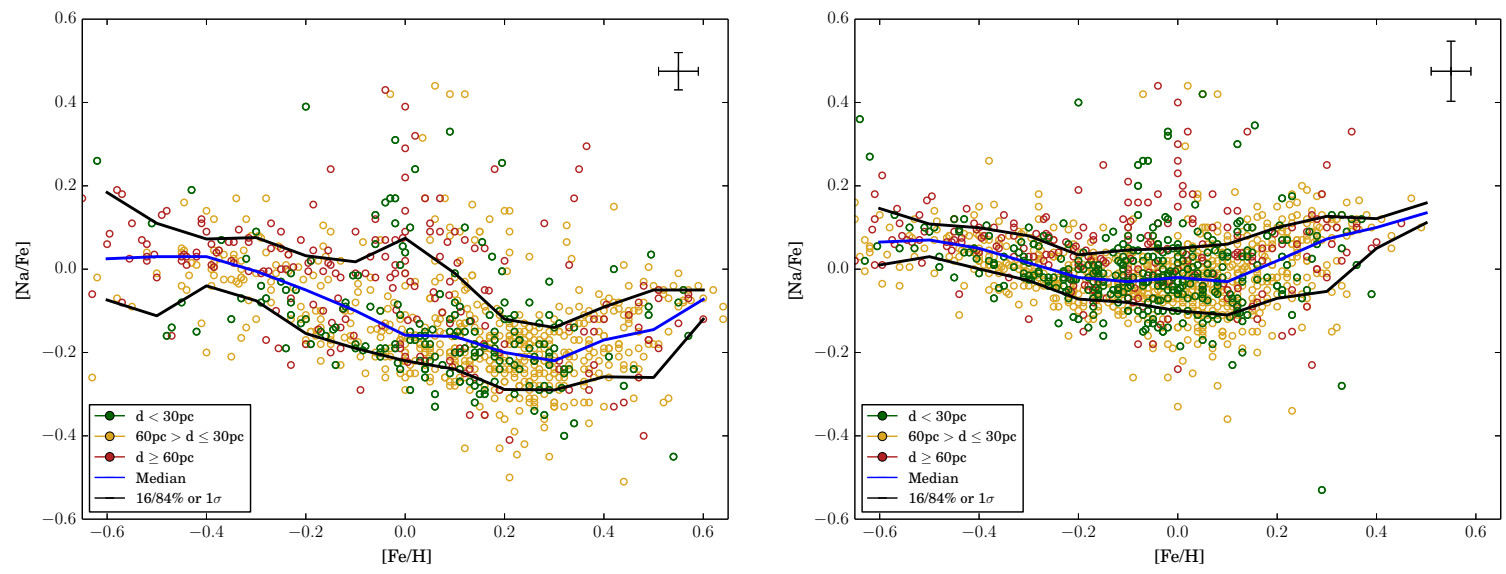

Fig. 13. - The $[\mathrm{Na} / \mathrm{Fe}]$ ratio as a function of $[\mathrm{Fe} / \mathrm{H}]$. The figure has the same format as Fig. 6 where the figure on the left shows $[\mathrm{Na} / \mathrm{Fe}]$ after the renormalization to Lodders et al. (2009) and the abundances on the right use the solar abundances determined by the literature sources.

The only stable isotope of sodium, ${ }^{23} \mathrm{Na}$, is produced mainly in carbon-burning in massive stars, whose final abundance is sensitive to the overall neutron enrichment (Woosley \& Weaver 1995 Chieffi \& Limongi 2004). The abundance ratio [Na/Fe], after being renormalized to a standard solar abundance (see 33.2 , as a function of the $[\mathrm{Fe} / \mathrm{H}]$ ratio is shown in Fig. 13 (left) for the 907 stars in the analysis of the Hypatia Catalog, see \$4 The median and $1 \sigma$ lines shows a shallow decreasing trend that curves up at higher $[\mathrm{Fe} / \mathrm{H}]$ metallicities, similar to that seen in Edvardsson et al. (1993); Bensby et al. (2003, 2005). The minimum of the median trend occurs below solar at $[\mathrm{Na} / \mathrm{Fe}] \approx-0.2$ dex and $[\mathrm{Fe} / \mathrm{H}] \approx 0.2$ dex, such that most of the stars observed have sub-solar $[\mathrm{Na} / \mathrm{Fe}]$ abundances. The majority of stellar abundances for $[\mathrm{Fe} / \mathrm{H}]>0.2$ dex came from both Neves et al. (2009); Valenti \& Fischer (2005), reinforcing the positive slope in this region seen in smaller numbers from other surveys. Overall, the $1 \sigma$ scatter increases from 0.13 dex at $[\mathrm{Fe} / \mathrm{H}]=$ -0.4 dex to 0.19 dex at $[\mathrm{Fe} / \mathrm{H}]=0.4 \mathrm{dex}$, see Table 4 . However, there is an increased amount of scatter in $[\mathrm{Na} / \mathrm{Fe}]$ for $[\mathrm{Fe} / \mathrm{H}]=[0.0,0.2]$ dex, note the $1 \sigma$ percentile is 0.26 at $[\mathrm{Fe} / \mathrm{H}]=0.0$ dex per 
Table 4. Those stars with $[\mathrm{Na} / \mathrm{Fe}]>0.2$ dex are predominantly from Thevenin (1998); Gebran et al. (2010), with a few contributions from Luck \& Heiter (2006); Valenti \& Fischer (2005); Edvardsson et al. (1993); Takeda (2007); Reddy et al. (2006); Feltzing \& Gustafsson (1998).

Fig. 13 (right) shows the effects of the individual abundance scales applied by each of the datasets. While there are some changes to the scatter between the two figures (left and right), the more noticeable distinction is the how the overall trend in $[\mathrm{Na} / \mathrm{Fe}]$ to $[\mathrm{Fe} / \mathrm{H}]$ to the left is more scattered compared to the right (see Table 4). As discussed for Fig. 6, the variation in abundance slopes in Fig. 13 indicates that the solar abundance scales are an important factor when comparing datasets (see Table 3). The significant shifting, where the mean and median absolute difference between the un-normalized and re-normalized [ $\mathrm{Na} / \mathrm{Fe}]$ abundances were 0.18 and 0.16 dex, respectively, causes stars to reposition themselves in new regions of the plot, which then varies the underlying trends. Using the same solar abundance scale is only one step out of many that need to be taken to accurately compare datasets between groups.

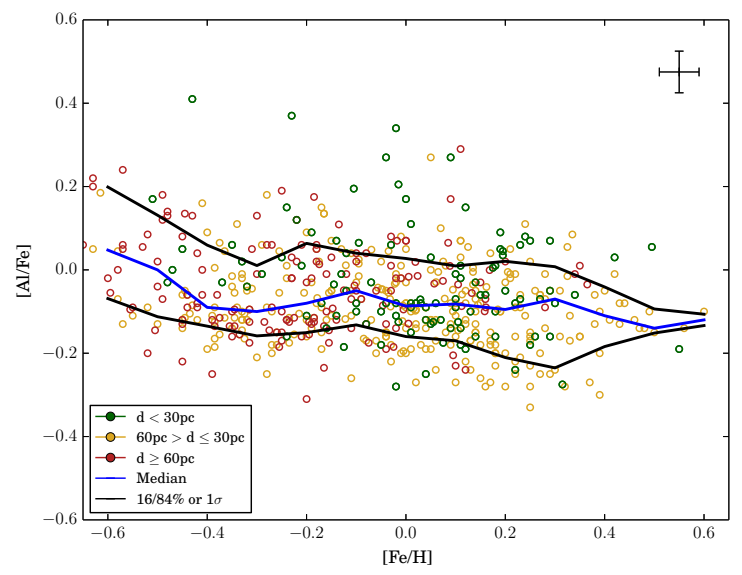

Fig. 14.- Similar to Fig. 5 but for aluminum.

Aluminum, whose only stable isotope is ${ }^{27} \mathrm{Al}$, is mainly synthesized in hydrostatic carbon and neon burning (Arnett \& Thielemann 1985; Thielemann \& Arnett 1985; Woosley \& Weaver 1995. Limongi \& Chieffi 2006). Evolution of $[\mathrm{Al} / \mathrm{Fe}]$ with $[\mathrm{Fe} / \mathrm{H}]$ is shown in Fig. 14 for the 523 stars in the analysis of the Hypatia Catalog, see $\$$. The three trend lines suggest $[\mathrm{Al} / \mathrm{Fe}]$ values are nearor-below solar with a shallow decreasing trend, consistent with the trends seen in data studies over a larger metallicity range (Peterson 1981; Magain 1989; Fulbright 2000; Brewer \& Carney 2006). For the majority of $[\mathrm{Fe} / \mathrm{H}]$, the $1 \sigma$ scatter in $[\mathrm{Al} / \mathrm{Fe}]$ is relatively constant (see Table 4). Although, curiously, there is a relative paucity of $[\mathrm{Al} / \mathrm{Fe}]$ measurements for stars with $[\mathrm{Fe} / \mathrm{H}] \gtrsim 0.4 \mathrm{dex}$. The few outlier stars with $[\mathrm{Al} / \mathrm{Fe}]$ above 0.2 dex are from a wide range of catalogs.

Fig. 15 shows $[\mathrm{Al} / \mathrm{Fe}]$ with respect to radial distance, colored to show height above the Galactic plane (left) and directionality towards or away from the galactic center (right). Stars within $60 \mathrm{pc}$ 
span a slightly larger range of $[\mathrm{Al} / \mathrm{Fe}],-0.3 \lesssim[\mathrm{Al} / \mathrm{Fe}] \lesssim 0.4$, than stars at larger distances. But this variation is on par with the error bars associated with $[\mathrm{Al} / \mathrm{Fe}]$. Using aluminum as a typical odd-Z element, there does not appear to be any distinct correlation between abundance and location in the local galaxy.
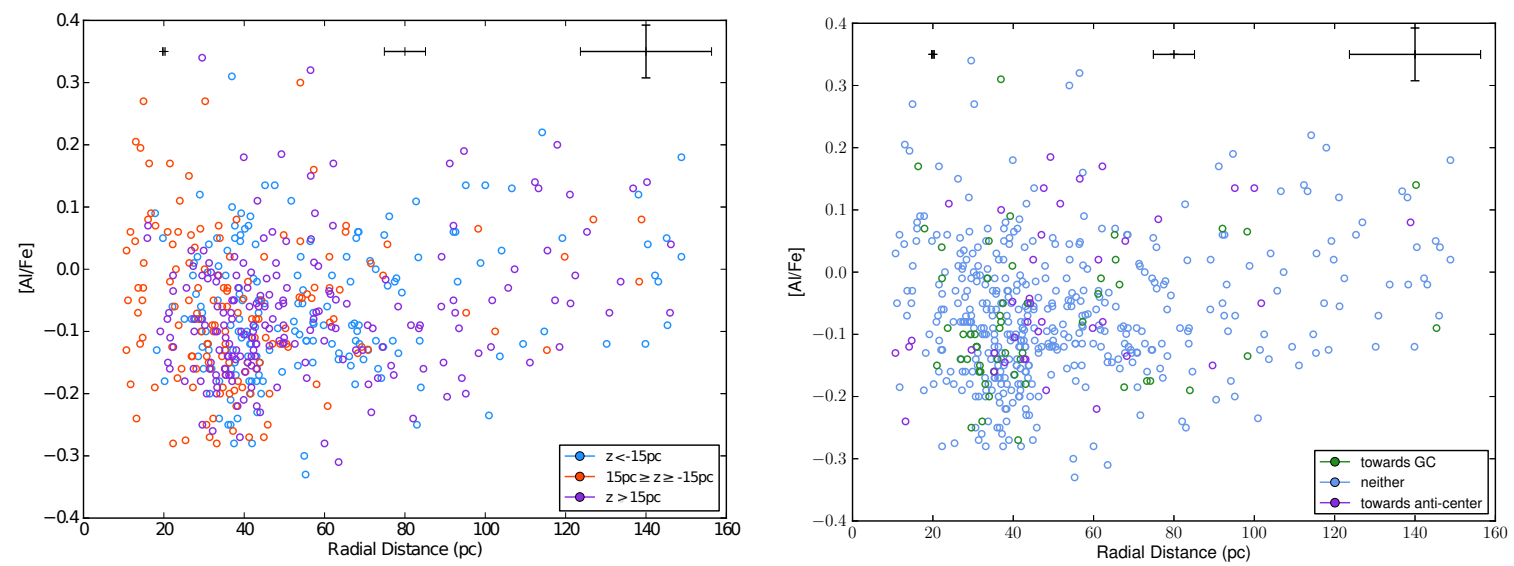

Fig. 15. - $[\mathrm{Al} / \mathrm{Fe}]$ ratio as a function of radial distance, similar to to Fig. 10 .

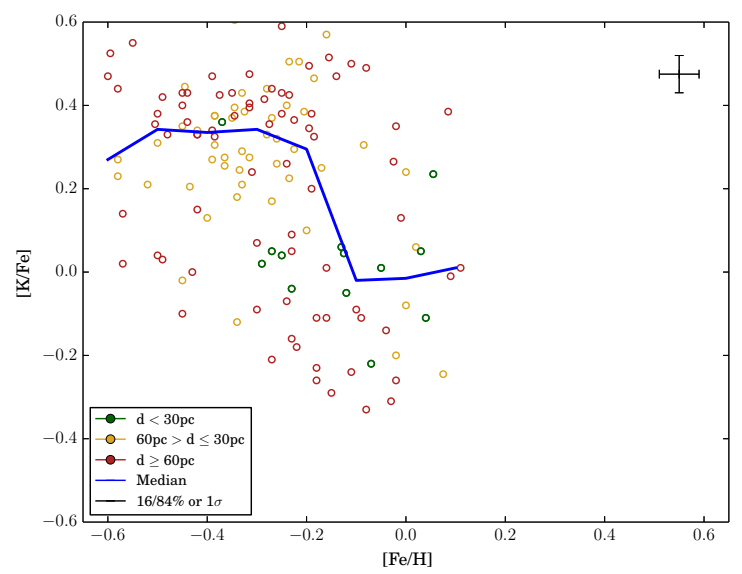

Fig. 16. - Similar to Fig. 5 but for potassium. Due to small number statistics, the percentile trend lines could not be accurately determined for [K/Fe], which has less than 250 stellar measurements (see Table 5).

The isotopes ${ }^{39,41} \mathrm{~K}$ are produced during oxygen burning, with ${ }^{41} \mathrm{~K}$ made as ${ }^{41} \mathrm{Ca}$, but some ${ }^{41} \mathrm{~K}$ is produced as itself during neon burning. The abundance ratio $[\mathrm{K} / \mathrm{Fe}]$ versus the $[\mathrm{Fe} / \mathrm{H}]$ ratio is shown in Fig. 16 for the 139 stars in the analysis of the Hypatia Catalog, see $\$ 4$. Blending between the $\mathrm{K} \mathrm{I}$ and atmospheric $\mathrm{O}_{2}$ lines means potassium abundances are more difficult to determine 
Gratton \& Sneden 1987), accounting for the relatively fewer number of stars with K abundance determinations. In addition, the low excitation energies of K I might be susceptible to non-LTE or strong hyperfine structure effects (Ivanova \& Shimanskil 2000). Despite the median blue line in Fig. 16 , the large scatter in $[\mathrm{K} / \mathrm{Fe}]$ makes it difficult to identify any sort of general trend. The only point of interest from Fig. 16 is the complete lack of stars with $[\mathrm{K} / \mathrm{Fe}]$ abundances at $[\mathrm{Fe} / \mathrm{H}]$ $>0.11$, despite the 6 data sets within Hypatia that have measured potassium (see Table 2).

A number of abundance determinations from Reddy et al. (2003) are used in Fig. 13 (right) and Fig. 16. They investigated 27 elements, including sodium and potassium, in $181 \mathrm{~F}$ and $\mathrm{G}$ dwarfs from a differential LTE analysis of high-resolution $(\Delta \lambda / \lambda \approx 60,000)$ and high signal-tonoise ( $\mathrm{S} / \mathrm{N}=300-400)$ spectra from the Smith $2.7 \mathrm{~m}$ telescope at McDonald Observatory. Of these 181 stars, 179 are in Hypatia. Effective temperatures were adopted from an infrared flux calibration of Str̈omgren photometry. Surface gravities and stellar ages were determined from stellar evolution tracks and Hipparcos Catalogue parallaxes. The $6154.23 \AA$ and $6160.75 \AA$ lines of Na I and the $7698.98 \AA$ line of $\mathrm{K}$ I were used in the analysis, with oscillator strengths taken from Lambert \& Warner (1968).

The isotope ${ }^{45} \mathrm{Sc}$ is made as itself and as radioactive ${ }^{45} \mathrm{~T}$ (Tuli 2005$)$ in hydrostatic and explosive oxygen-burning and in alpha-rich freezeouts in core-collapse events (Rauscher et al. 2002; Limongi \& Chieffi 2003; Ekström et al. 2011). The trend of [Sc/Fe] versus [Fe/H] is shown in Fig. 17 for the 381 stars with Sc I measurements (left) in the analysis of the Hypatia Catalog, see $\$ 4$, and the 386 stars with Sc II abundance determinations (right). The $[\mathrm{Sc} / \mathrm{Fe}]$ ratios are relatively flat with respect to $[\mathrm{Fe} / \mathrm{H}]$, where the median of the abundance measurements is slightly above solar at low $[\mathrm{Fe} / \mathrm{H}]$ ratios and slightly below solar at higher metallicities (see Table 4). The $1 \sigma$ trend lines point out the small number of stars with $[\mathrm{Sc} / \mathrm{Fe}]$ measurements for $[\mathrm{Fe} / \mathrm{H}]<-0.2$ and $>0.4$. Their scatter around the solar iron ratio is 0.28 dex.

In contrast, $[\mathrm{Sc} \mathrm{II} / \mathrm{Fe}]$ ratios follow a more steeply decreasing trend with $[\mathrm{Fe} / \mathrm{H}]$ (Thevenin 1998; Zhang \& Zhao 2006). The small $1 \sigma$ scatter at either end of the $[\mathrm{Fe} / \mathrm{H}]$ extrema, 0.14 and 0.09 dex, respectively, is mitigated by the relatively large 0.23 dex scatter at the solar value of $[\mathrm{Fe} / \mathrm{H}]$. While a few stars of these stars also have $[\mathrm{ScII} / \mathrm{Fe}]$ above $0.3 \mathrm{dex}$, their measurements come from a number of different catalogs. The majority of the scatter may be dependent on whether individual studies correctly included hyperfine structure in their Sc II abundance determinations. To this end, we found that only 3 of the 15 catalogs, Feltzing \& Gustafsson (1998); Allende Prieto et al. (2004); Gebran et al. (2010), that measured Sc II ratios incorporated hyperfine structure in their analysis. Analyzing the [ScII/Fe] abundances from only these three catalog still produces some scatter. However, given that the total number of stars was 159 , we don't find our results to be conclusive.

Similar to the trends seen for $[\mathrm{Mg} / \mathrm{Fe}],[\mathrm{Si} / \mathrm{Fe}]$, and $[\mathrm{S} / \mathrm{Fe}]$ (see Figs. 5- 7), the stars for which $[\mathrm{ScII} / \mathrm{Fe}]$ was measured show two "ensembles" separated by a "gap" between $[\mathrm{ScII} / \mathrm{Fe}] \approx[0.0,0.1]$. Further investigation revealed that the majority of the lower "ensemble" was a result of the Neves 
et al. (2009) dataset. The inclusion of their catalog increased the number of stars with [ScII $/ \mathrm{Fe}]<$ -0.2 by an order of magnitude. The contribution of the additional, unique stars acted to solidify the trend that was already present from other datasets, although in smaller numbers. We therefore maintain that this "gap" is physical, as opposed to a signature of catalog compilation. In a similar vein as the discussion for $[\mathrm{Ca} / \mathrm{Fe}]$ in Fig. 8, we find that the predominant number of stars near to the Sun in the lower "ensemble" is a direct consequence of the Neves et al. (2009) dataset only observing stars at distances $<60 \mathrm{pc}$. For a more complete analysis, we would require additional abundance measurements for stars more than $60 \mathrm{pc}$ from the Sun or a standardized method for determining stellar abundances. We discuss the two "ensembles" seen in the $[\mathrm{ScII} / \mathrm{Fe}]$ vs. $[\mathrm{Fe} / \mathrm{H}]$ plot in $\$ 11$.

Feltzing \& Gustafsson (1998) explored scandium abundances in 47 G and K dwarf stars with -0.1 dex $<[\mathrm{Fe} / \mathrm{H}]<0.42$ dex using a differential LTE analysis with respect to the Sun of highresolution $(\Delta \lambda / \lambda \approx 100,000)$ and high signal-to-noise $(\mathrm{S} / \mathrm{N} \approx 200)$ spectra. Of these 47 stars, 45 are in Hypatia (see Table 22). The 5484.64 $\AA$ line is used for Sc I and the $5239.82 \AA 5318.36 \AA 6300.69$ $\AA$ $6320.84 \AA$ lines are used for Sc II. Noting that single line abundance determinations should be viewed with caution, Feltzing \& Gustafsson (1998) base their scandium abundance determinations on Sc II and discuss the apparent overionization and other non-LTE effects that may effect most of

$\mathrm{t}$

c

1

1

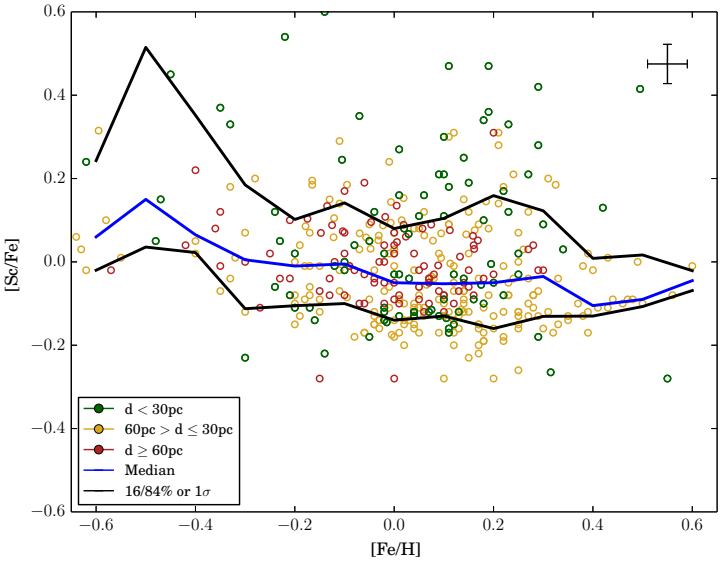

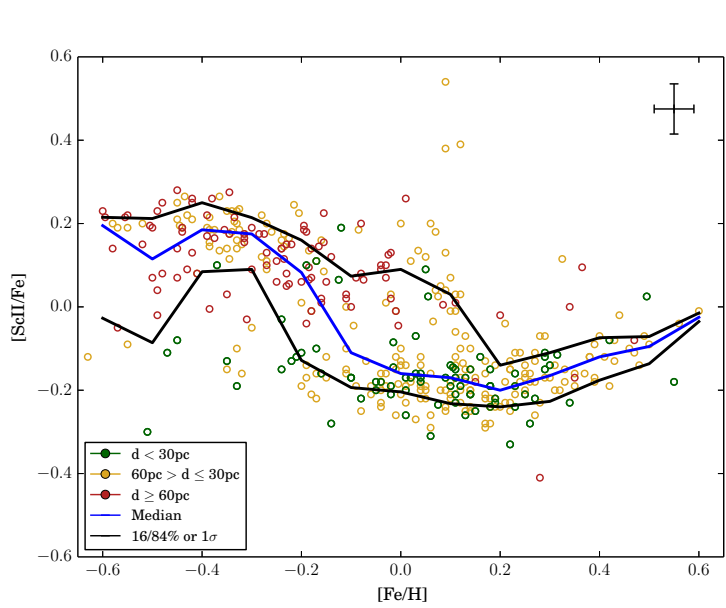

Fig. 17. - The $[\mathrm{Sc} / \mathrm{Fe}]$ ratio as a function of $[\mathrm{Fe} / \mathrm{H}]$, with the same format as Fig. 5 .

\section{Iron-Peak Elements ( V, Cr, Mn, Co, \& Ni)}

The elements V, Cr, Mn, Co, and Ni are formed by the same nuclear processes that create iron in core-collapse and thermonuclear supernova, in varying degrees (Thielemann et al. 2002; Limongi \& Chieffi 2003; Thielemann et al. 2007). Vanadium is dominated by the isotope ${ }^{51} \mathrm{~V}$, which is produced as ${ }^{51} \mathrm{Cr}$ and ${ }^{51} \mathrm{Mn}$ during explosive oxygen burning, explosive silicon burning, and $\alpha$-rich freezeouts in core-collapse supernovae (Clayton 2003). Chromium, essentially ${ }^{52} \mathrm{Cr}$, is formed as 
a result of radioactive decay from ${ }^{52} \mathrm{Fe}$ during quasiequilibrium explosive silicon burning Arnett 1996; Dauphas et al. 2010). Manganese - dominated by ${ }^{55} \mathrm{Mn}$ from the radioactive decay of ${ }^{55} \mathrm{Co}$, cobalt - dominated by ${ }^{59} \mathrm{Co}$ from the radioactive decay of ${ }^{59} \mathrm{Cu}$, and nickel - dominated by ${ }^{58} \mathrm{Ni}$ made as itself, are all generally the result of quasiequilibrium reactions during explosive silicon burning (Woosley \& Weaver 1995). Because of these elements' proximity to iron (see $\$ 5.1$ ), most of the abundance evolutions track iron. However, given the noted scatter for the elements in this subsection, there is a striking case for improving the oscillator strength values for the iron-peak abundances. All of the iron-group elements have been plotted with the same $\mathrm{x}$ - and y-axis scales, where $[\mathrm{Fe} / \mathrm{H}]=[-0.6,0.6]$ and $[\mathrm{X} / \mathrm{Fe}]=[-0.65,0.5]$, respectively.

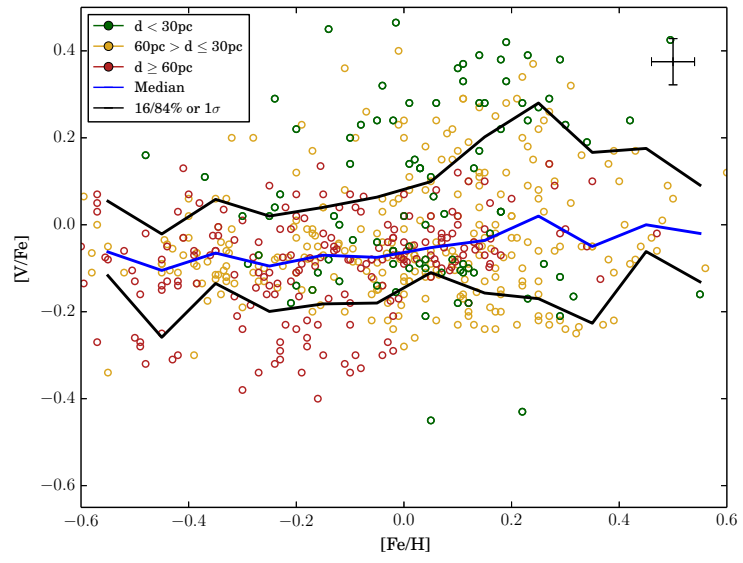

Fig. 18. - Same as Fig. 5 but for vanadium.

Of the 28 literature sources in the Hypatia Catalog that determined vanadium abundances, only Feltzing \& Gustafsson (1998) and Takeda (2007) determined vanadium abundances from both ionization states. Both surveys reported the lines for the neutral and ionized species were limited to only one or two lines in the optical spectrum, or too weak to separate out from the spectrum. Zhang et al. (2008) reported the vanadium abundances using V I, for 32 mildly metal poor stars using spectra with a signal-noise ratio of about 150 per pixel at $6400 \AA$ and a resolving power of about 37,000. Solar abundances, calculated from the daylight spectrum were used to derive stellar abundances relative to the Sun. The effective temperature was determined from the $b-y$ and $V-K$ color indices; surface gravities were calculated from Hipparcos parallax. They reported that V I follows Fe very closely, with no offset between thin and thick disk stars.

The ratio $[\mathrm{V} / \mathrm{Fe}]$ versus $[\mathrm{Fe} / \mathrm{H}]$ is shown in Fig. 18 for the 564 stars in the analysis of the Hypatia Catalog, see $₫ 4$. The median $[\mathrm{V} / \mathrm{Fe}]$, shown by the blue line, indicates a flat and slightly sub-solar trend for all $[\mathrm{Fe} / \mathrm{H}]$ content, which is consistent with its nucleosynthetic origin. The average scatter, as determined by the $1 \sigma$ percentile lines, is relatively large: 0.22 dex (see Table 4). This scatter was persistent across many individual datasets and may be the result of hyperfine structure being treated rather "casually" for the V I lines. 

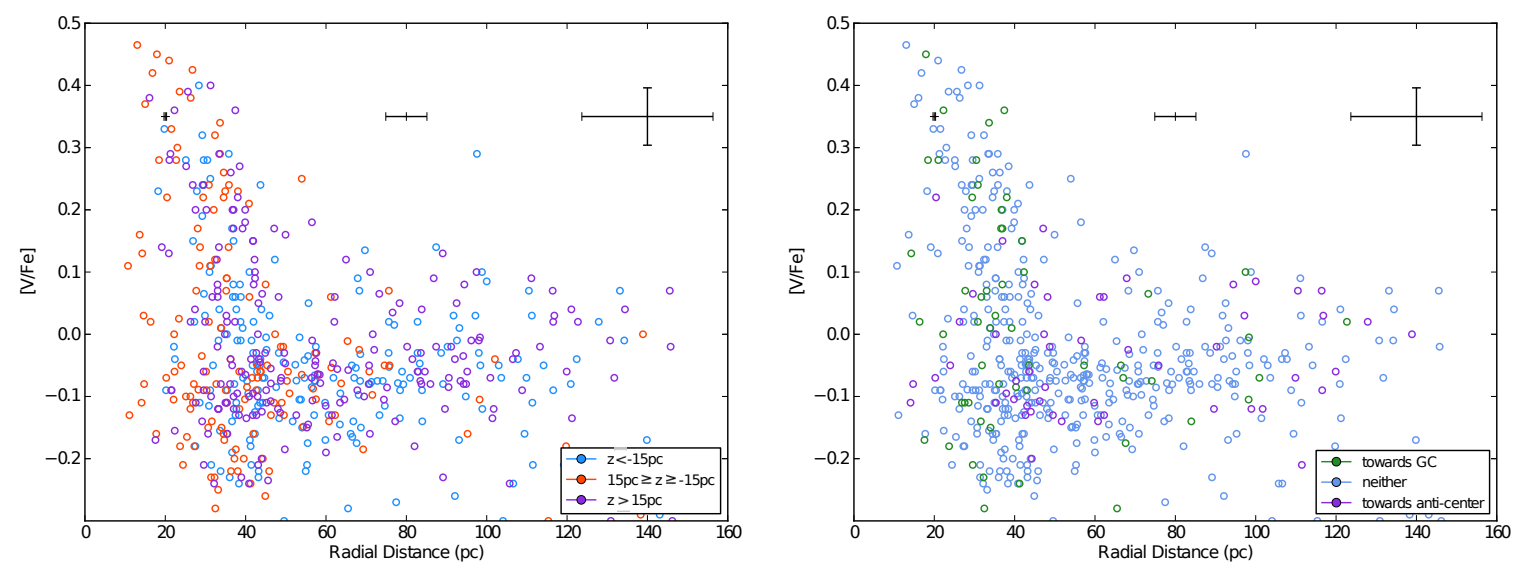

Fig. 19.- Similar to Fig. 10, but for vanadium.

Fig. 19 shows $[\mathrm{V} / \mathrm{Fe}]$ ratios versus radial distance, colored to show the height above the Galactic plane (left plot) and directionality towards or away from the galactic center (right plot). There is a large scatter of $\approx 0.8 \mathrm{dex}$ in $[\mathrm{V} / \mathrm{Fe}]$ for those stars with a distance less than $\sim 40 \mathrm{pc}$ and away from the galactic center (blue and green circles). The large scatter at radial distances less than $40 \mathrm{pc}$ is an interesting and unexpected result. The majority of abundances beyond $40 \mathrm{pc}$ lie below $[\mathrm{V} / \mathrm{Fe}]=$ 0.1 dex, however near to the Sun $[\mathrm{V} / \mathrm{Fe}]=[-0.3,0.5]$. In addition, stellar abundances greater than 0.1 dex come from a variety of different surveys. Because there are a number of unblended V I lines in the optical spectrum, we do not believe this cutoff is a function of survey or instrument bias. For now, we leave this question as something to examine more thoroughly in the future. To the right of Fig. 19, there were fewer stars for which $[\mathrm{V} / \mathrm{Fe}]$ was measured near the galactic plane at large radial distances. However, it does appear that stars towards the Galactic anti-center (purple) tend to cluster around solar $[\mathrm{V} / \mathrm{Fe}]$ at all distances.

Evolution of $[\mathrm{Cr} / \mathrm{Fe}]$ with $[\mathrm{Fe} / \mathrm{H}]$ is shown in Fig. 20 for the 483 stars in the analysis of Hypatia Catalog with both neutral Cr abundances determinations (left) and the 329 stars with Cr II based abundance determinations (right). Thirty-one catalogs within Hypatia report abundances from either pure Cr I lines or a blend with Cr II, while nine surveys published using only Cr II lines (see Table 2). For example, Neves et al. (2009) present a survey 12 elements whose abundances are derived from spectra obtained with the HARPS spectrograph on the ESO $3.6 \mathrm{~m}$ telescope. The $\mathrm{Cr}$ I lines $4588.20 \AA$ and $4592.05 \AA$ along with the Cr II line of $4884.61 \AA$ were used in a differential LTE analysis relative to the Sun to determine the abundance levels. Of the 451 stars in the Neves et al. (2009) survey, 443 are in the Hypatia Catalog. Initial estimates of the oscillator strengths were taken from the Vienna Atomic Line Database and refined using a semi-empirical, inverse analysis with the MOOG2002 (Sneden 1973). Effective temperatures, surface gravity, microturbulence, and metallicity were taken from Sousa et al. (2008). Neves et al. (2009) reported that abundance levels determined from neutral states are more sensitive to effective temperature changes, whereas 
abundances derived from ionized states are more sensitive to changes in surface gravity. Abundances from ionized elements are also more sensitive to metallicity changes than the neutral elements, although the sensitivity is not as significant as for the effective temperature or surface gravity.
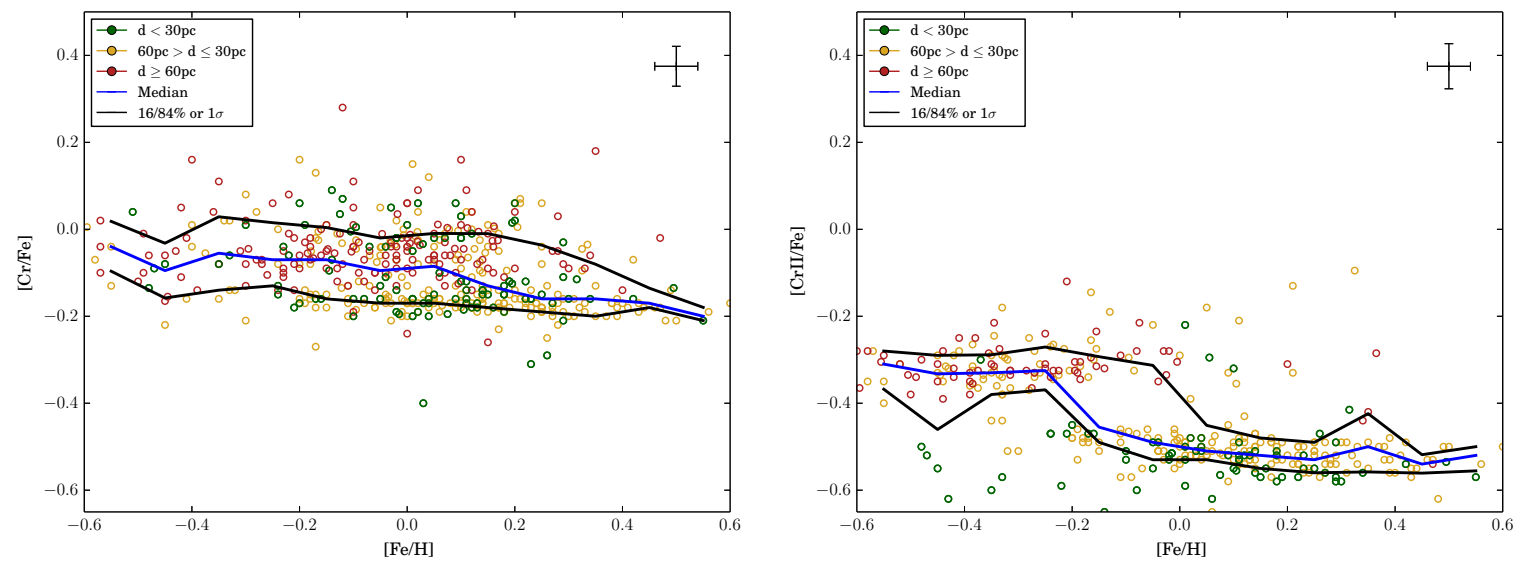

Fig. 20.— Same as Fig. 5 but for neutral (left) and ionized (right) chromium.

While most of the stars in Fig. 20 for both ionization states are below solar, the trend for $[\mathrm{Cr} / \mathrm{Fe}]$ is near 0.0 dex with a number of stars beyond $1 \sigma$ above the solar value of $[\mathrm{Cr} / \mathrm{Fe}]=$ 0.0 dex. The $1 \sigma$ scatter in $[\mathrm{Cr} / \mathrm{Fe}]$ is relatively constant, around 0.18 dex, for $[\mathrm{Fe} / \mathrm{H}]<0.2 \mathrm{dex}$, where the number of stars observed at higher metallicities severely decreases. Comparatively, the scatter in $[\mathrm{Cr} \mathrm{II} / \mathrm{Fe}]$ is roughly half of that for the neutral state, please reference Table 4 , while the abundances for [Cr II/Fe] are markedly lower. As discussed in Neves et al. (2009), [Cr II/Fe] has a slight downward trend with increasing $[\mathrm{Fe} / \mathrm{H}]$, with a corresponding weak trend for $[\mathrm{Cr} / \mathrm{Fe}]$. These differences may be the result of weak, blended Cr II lines (Neves et al. 2009), different surface gravities for the two ionization states (Reddy et al. 2003; Gratton et al. 2003), or overionization from Cr II (Feltzing \& Gustafsson 1998).

Noticeable in both plots in Fig. 20 is an "ensemble" of stars the show a depletion in the Cr $\mathrm{I} / \mathrm{II}$ ratio and increased $[\mathrm{Fe} / \mathrm{H}]$. A significant fraction of stars that are within $60 \mathrm{pc}$ of the Sun (green and yellow circles) appear to be clustered around $[\mathrm{Cr} / \mathrm{Fe}] \approx-0.2$ dex and and below a gap at -0.4 dex for $[\mathrm{Cr} \mathrm{II} / \mathrm{Fe}]$. Further investigation into this phenomena has found many of these measurements originated from Neves et al. (2009), without which the higher $[\mathrm{Fe} / \mathrm{H}]$ measurements and lower Cr I/II abundance ratio regime would be sparsely populated. Similar to a number of elements already discussed, like silicon, sulfur, and ionized scandium, the $[\mathrm{Cr} \mathrm{II} / \mathrm{Fe}]$ vs. $[\mathrm{Fe} / \mathrm{H}]$ plot shows a dual ensemble of stars, separated by $\sim 0.1$ dex around $[\mathrm{Cr}$ II $/ \mathrm{Fe}]=-0.4$ dex (see $\$ 11$ for more discussion).

Variation of the $[\mathrm{Mn} / \mathrm{Fe}]$ ratio with $[\mathrm{Fe} / \mathrm{H}]$ for the 512 stars in the analysis of Hypatia is shown in Fig. 21 (left). A unique element in the iron group, the manganese and iron ratio is shown to 

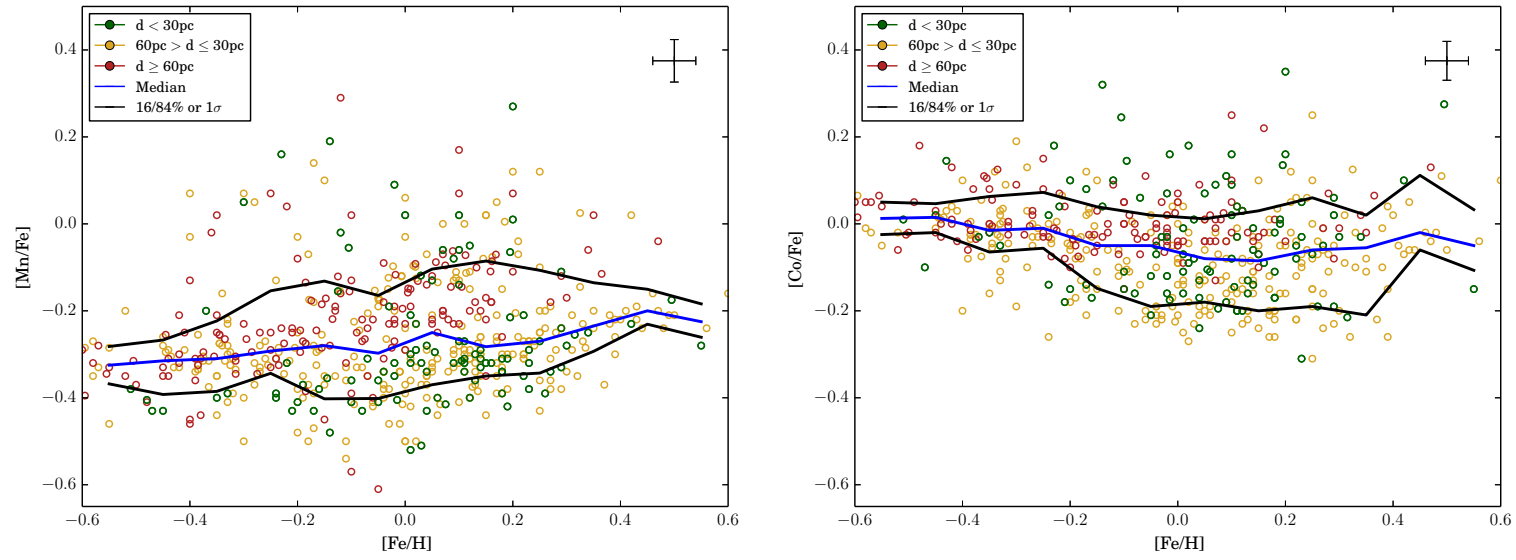

Fig. 21.- Same as Fig. 5 but for manganese (left) and cobalt (right).

slightly increase with $[\mathrm{Fe} / \mathrm{H}]$ (Helfer et al. 1959, Gratton 1989, Goswami \& Prantzos 2000; Feltzing et al. 2007). The $1 \sigma$ scatter in $[\mathrm{Mn} / \mathrm{Fe}]$ is large and variable, going from 0.17 dex at $[\mathrm{Fe} / \mathrm{H}]=$ -0.4 dex, to 0.27 dex at solar $[\mathrm{Fe} / \mathrm{H}]$, and then down again to $0.08 \mathrm{dex}$ at $[\mathrm{Fe} / \mathrm{H}]=0.4 \operatorname{dex}$ (see Table 4). The majority of the scatter above the $84 \%$ percentile regression line, particularly [Mn/Fe] $>0.0$ dex, is largely from Thevenin (1998), with a few contributions from other data sets. The slightly increasing median trend line, coupled with notable abundance corrections from hyperfine splitting effects in strong lines, has made the rise in $[\mathrm{Mn} / \mathrm{Fe}]$ with $[\mathrm{Fe} / \mathrm{H}]$ challenging to decipher. Manganese could have formed from either core collapse supernovae or SN Ia, but it is difficult to tell which is dominating the trend for $[\mathrm{Fe} / \mathrm{H}]>-1.0$ dex (Chen et al. 2000; Prochaska \& McWilliam 2000; McWilliam et al. 2003; Bergemann \& Gehren 2007; Feltzing et al. 2007; Bergemann \& Gehren 2008).

Fig. 22 shows $[\mathrm{Mn} / \mathrm{Fe}]$ ratios versus radial distance, colored to show the height above the Galactic plane (left) and directionality towards or away from the galactic center (right). There is scatter in $[\mathrm{Mn} / \mathrm{Fe}], \approx 0.7 \mathrm{dex}$, for stars that are nearer to the Sun, although at further distances the scatter decreases to $\approx 0.5 \mathrm{dex}$. Interestingly, stars near to the Sun, or within $\sim 30 \mathrm{pc}$, that have $[\mathrm{Mn} / \mathrm{Fe}]>-0.2$ dex tend to be closer to the Galactic plane (red circles) than the majority of stars, with $[\mathrm{Mn} / \mathrm{Fe}]<-0.2$ dex. There is a relative dearth of stars well above or below the plane with more enriched $[\mathrm{Mn} / \mathrm{Fe}]$ ratios. Whether this is a result of survey biases or a physical causation remains to studied further. Stars that are towards the galactic anti-center (purple circles) are more enriched in $[\mathrm{Mn} / \mathrm{Fe}]$ than the other neighboring stars, with few of them exhibiting abundances below -0.4 dex, especially at nearby radial distances.

The single stable isotope of cobalt, ${ }^{59} \mathrm{Co}$, is produced by a variety of processes in several sources; as a result, there is no consensus on the overall trend of cobalt in halo, thick, or thin disk stars, nor a generally accepted production origin site (Bergemann et al. 2010). Variation of 

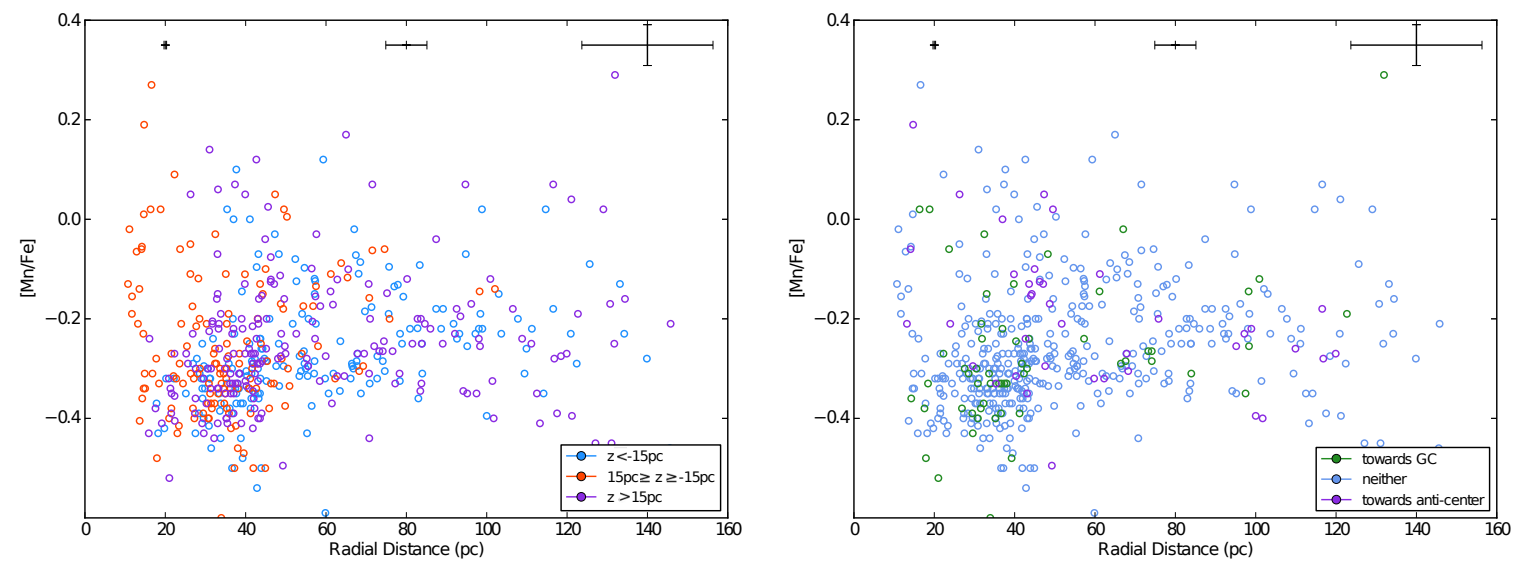

Fig. 22. - Like Fig. 10 but for manganese.

the $[\mathrm{Co} / \mathrm{Fe}]$ ratio with $[\mathrm{Fe} / \mathrm{H}]$ is shown in Fig. 21 (right). The median $[\mathrm{Co} / \mathrm{Fe}]$ ratio indicates a relatively flat trend, that goes slightly concave-up for higher $[\mathrm{Fe} / \mathrm{H}]$ abundances. This feature is made more pronounced with the inclusion of the $1 \sigma$ regression lines, and has noted by several authors (Reddy et al. 2003, del Peloso et al. 2005; Reddy et al. 2006, Neves et al. 2009). For [Fe/H] below solar, $[\mathrm{Co} / \mathrm{Fe}]$ decreases from $\approx 0.0$ dex down about 0.1 dex, with a scatter varying from $\approx$ 0.1 dex to $\approx 0.2$ dex (see Table 4 ). When $[\mathrm{Fe} / \mathrm{H}]$ is above solar, $[\mathrm{Co} / \mathrm{Fe}]$ increases very slightly, although remaining below solar, with a scatter of $\approx 0.15$ dex. Similar to $[\mathrm{Mn} / \mathrm{Fe}]$ (left), stars that are at larger distances above $60 \mathrm{pc}$ are clustered around the solar value of $[\mathrm{Co} / \mathrm{Fe}]$, with few stars at greater distances measured below -0.1 dex, giving cause to further abundance surveys for stars at $60 \mathrm{pc}$ or greater.

Nickel has the most measurements of any element within the iron group in Hypatia; forty literature sources are listed with $[\mathrm{Ni} / \mathrm{Fe}]$ abundances in Table 2 . This is because nickel has a similar ionization potential and atomic structure as iron and is relatively easy to measure in the optical spectrum. For example, Gilli et al. (2006) determined the abundances nickel (and 11 other elements) for 101 stars in the solar neighborhood, 93 of which are known to be host-stars to exoplanets. A total of 98 of their stars are in the Hypatia Catalog. Their spectra were acquired using five different spectrographs that, in total, spanned the range of $3800 \AA$ to $10000 \AA$, with significant overlap in wavelength coverage between the spectrographs. The maximum resolution was $\lambda / \Delta \lambda \approx 110000$ and minimum resolution of $\lambda / \Delta \lambda \approx 48000$. A standard LTE analysis, with respect to the solar abundances determined by Anders \& Grevesse (1989), was done for all elements using MOOG (Sneden 1973) and the ATLAS9 atmospheres (Kurucz 2005). Effective temperatures, surface gravities, microturbulence, and metallicity $[\mathrm{Fe} / \mathrm{H}]$ were all determined by Santos et al. (2005. 2004). The spectral lines that were used for refractory elements matched those within Bodaghee et al. (2003), while the lines for the other elements are from Beirão et al. (2005). Gilli et al. (2006) estimate an overall uncertainty of of $\sim 0.10$ dex for all abundance determinations. 

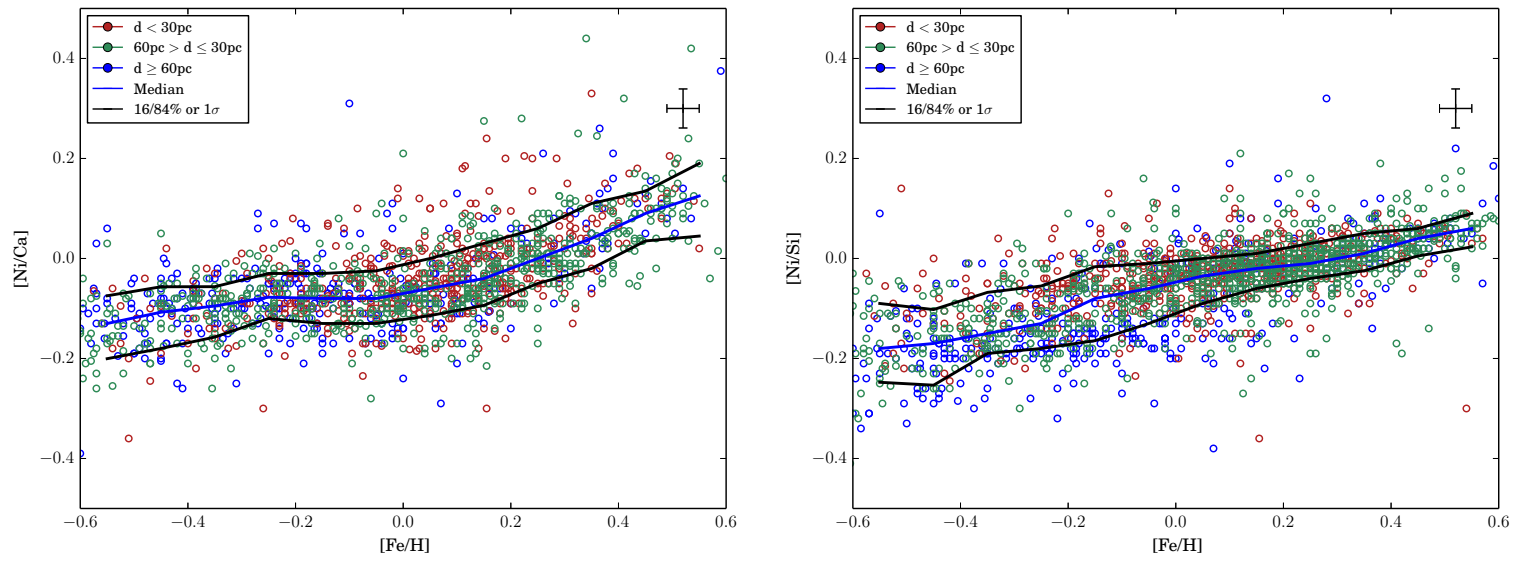

Fig. 23. - Similar to Fig. 11 but for the $\alpha$-chain elements [Ni/Ca] (left) and [Ni/Si] (right) plotted against $[\mathrm{Fe} / \mathrm{H}]$.

Fig. 23 shows the evolution of $[\mathrm{Ni} / \mathrm{Ca}]$ (left) and $[\mathrm{Ni} / \mathrm{Ca}]$ (right) with $[\mathrm{Fe} / \mathrm{H}]$. Both show positively sloped trends with $[\mathrm{Fe} / \mathrm{H}]$, indicating the injection of the iron-peak nuclei from SN Ia for all $[\mathrm{Fe} / \mathrm{H}]$ abundance measurements. The scatter of $[\mathrm{Ni} / \mathrm{Ca}]$ is $\approx 0.2$ dex, with a very small percentage of stars above or below the 16-84\% quantile regressions lines. In comparison, $[\mathrm{Ni} / \mathrm{Si}]$ (Fig. 23, right) has a scatter of $\sim 0.2$ dex for $[\mathrm{Fe} / \mathrm{H}] \lesssim 0.0 \mathrm{dex}$, which tapers to $\sim 0.1$ dex for $[\mathrm{Fe} / \mathrm{H}]$ above solar. In both plots, there is relatively small scatter about the median trend lines (blue). From Fig. 1, it is clear that all three elements are highly measured in the Hypatia Catalog. Looking at the individual abundance plots for Si (Fig. 7), Ca (Fig. 8), and Ni, (Fig. 24), we see that all three have small $1 \sigma$ scatter in their own regard (Table 4). Examining the correlations in Fig. 23 provides a stringent test of the Hypatia methodology. These elements are all well measured in many stars by many surveys. The spectra have numerous, strong, unblended lines in the optical, and so are less prone to systematic differences in derived abundances with different measurement techniques. Theoretically, they are expected to have a relatively tight correlation. Any errors in the methodology of combining, renormalizing, compiling, or with respect to making kinematic or other cuts to the data would be most easily detected in departures from these correlations. This gives us more confidence in the robustness of trends, such as those discussed in $\$ 11$.

Evolution of the $[\mathrm{Ni} / \mathrm{Fe}]$ ratio with $[\mathrm{Fe} / \mathrm{H}]$ for the 1035 stars in the Hypatia analysis is shown in Fig. 24. The $1 \sigma$ scatter evolves from $\sim 0.05$ dex to $\sim 0.20$ dex to $\sim 0.10$ dex, per Table 4 , as metallicity increases. Many stars cluster near the solar value of $[\mathrm{Ni} / \mathrm{Fe}]$, as well as $[\mathrm{Ni} / \mathrm{Fe}]=-0.2$ dex. We note that small group of stars between $[\mathrm{Ni} / \mathrm{Fe}]=[0.0,0.2]$ for $[\mathrm{Fe} / \mathrm{H}]>0.0$ dex is mostly from the Trevisan et al. (2011) dataset. Similar to the other elements displaying a "gap" in their abundance plots (Mg, Si, S, ScII, and $\mathrm{CrII})$, we note that the ensembles are spatially dependent, such that the stars further away are more enriched in $[\mathrm{Ni} / \mathrm{Fe}]$. The lower "ensemble" stars, at radial distances less than 60 pc, originate from a number of different datasets, including Valenti \& Fischer 
(2005). See $\$ 11$ for more discussion.

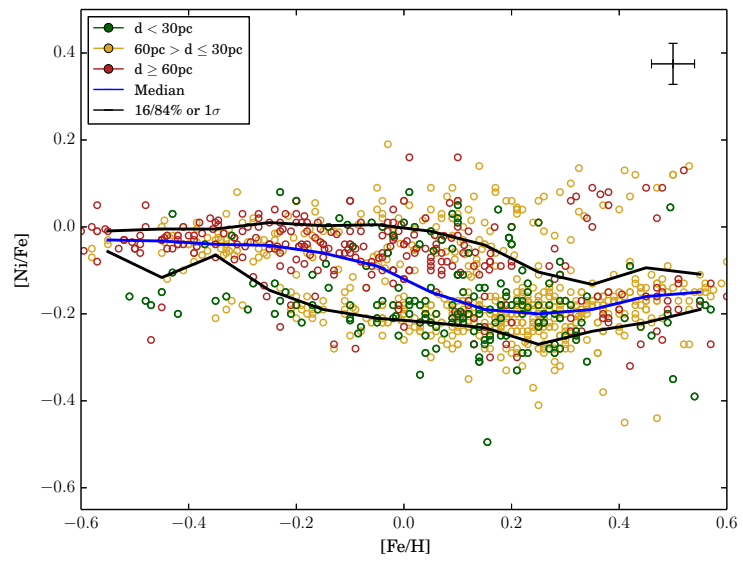

Fig. 24.- Same as Fig. 5 but for nickel.

\section{Beyond the Iron-Peak (Cu, Zn, Sr, Y, \& Zr)}

Nuclei above the iron peak have large Coulomb barriers, making charged-particle interactions unlikely at temperatures that would not photodissintegrate the nuclei. These elements also have fewer spectral lines in the optical regime, resulting in fewer abundance determinations as shown in Fig. 1. As a consequence, the $\mathrm{x}$ - and $\mathrm{y}$-axis scales for the elements beyond the iron-peak show a wider range compared with the previous elements, such that $[\mathrm{Fe} / \mathrm{H}]=[-0.7,0.4]$ and $[\mathrm{X} / \mathrm{Fe}]=$ $[-0.5,1.0]$, respectively.

Copper has two stable isotopes, ${ }^{63} \mathrm{Cu}$ and ${ }^{65} \mathrm{Cu}$; the more common isotope, ${ }^{63} \mathrm{Cu}$, is mostly created as radioactive ${ }^{63} \mathrm{Ni}$ via the s-process during hydrostatic helium burning in massive stars and intermediate mass AGB stars. The evolution of $[\mathrm{Cu} / \mathrm{Fe}]$ with $[\mathrm{Fe} / \mathrm{H}]$, as shown in Fig. 25 (left), is mostly constant and sub-solar with some scatter about the blue median line. The only exception are the two outlier stars that have $[\mathrm{Cu} / \mathrm{Fe}]>0.4$ dex; namely, HIP 78680 and HIP 64497, both measured by Ramírez et al. (2009). Because the low number of stars for which $[\mathrm{Cu} / \mathrm{Fe}]$ was measured, particularly at lower $[\mathrm{Fe} / \mathrm{H}]$ abundances, the $1 \sigma$ quantile regression lines could not be accurately determined.

Zinc is made as radioactive ${ }^{64} \mathrm{Ge}$ in the s-process during hydrostatic helium burning Couch et al. 1974; Iben 1982; Busso et al. 1999; Käppeler et al. 2011) and in $\alpha$-rich freezeouts from massive stars (Woosley et al. 1973; Hix \& Thielemann 1996), but consensus on the origin site has not yet been reached (Hoffman et al. 1996; Umeda \& Nomoto 2002; Chen et al. 2004; Mishenina et al. 2011a). The evolution of $[\mathrm{Zn} / \mathrm{Fe}]$ with $[\mathrm{Fe} / \mathrm{H}]$, as shown in Fig. 25 (right), has a relatively flat trend, punctuated by a small number of measurements at the extreme $[\mathrm{Fe} / \mathrm{H}]$ metallicities. 

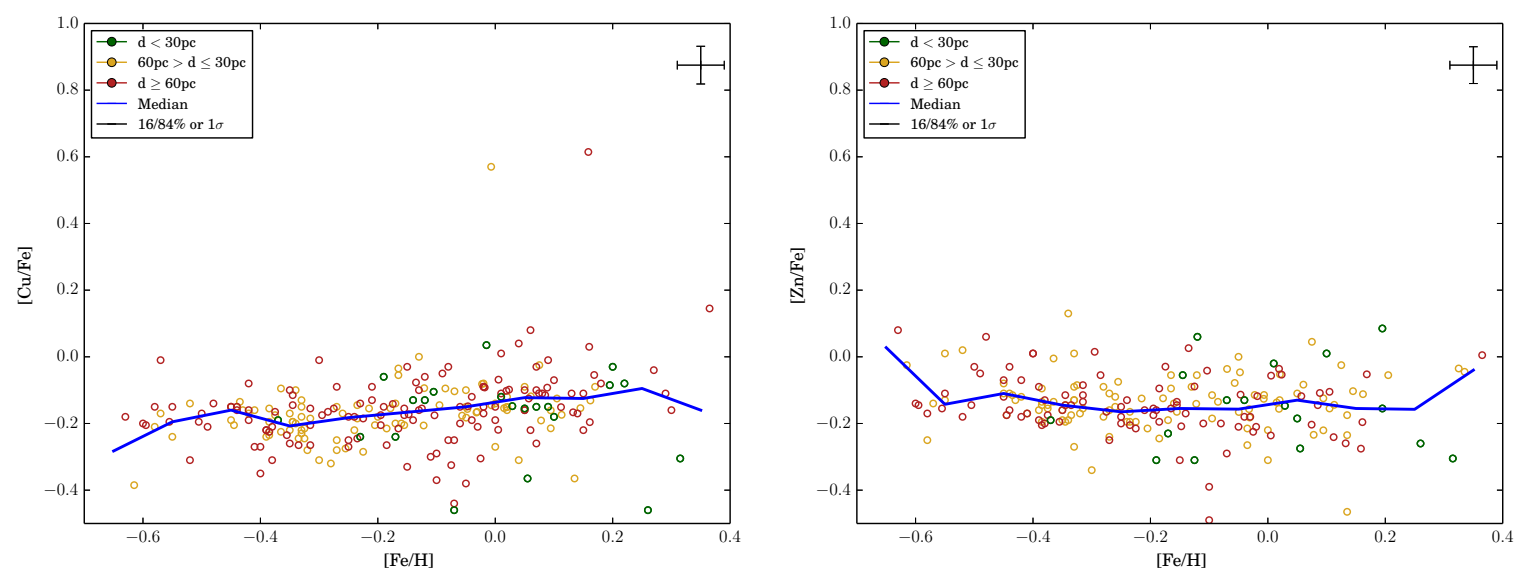

Fig. 25. - Same as Fig. 5 but for copper (left) and zinc (right). Due to small number statistics, the percentile trend lines could not be accurately determined for $[\mathrm{Cu} / \mathrm{Fe}]$ and $[\mathrm{Zn} / \mathrm{Fe}]$, which has less than 250 stellar measurements (see Table 5). Also note the y-axis scale change.

Noted also by Roederer et al. (2010a); Kobayashi \& Nakasato (2011), the majority of the [Zn/Fe] abundances are sub-solar. This is particularly interesting given the markedly non-solar abundances also seen in $[\mathrm{Cu} / \mathrm{Fe}]$ with respect to $[\mathrm{Fe} / \mathrm{H}]$, shown in Fig. 25 (left). The sub-solar patterns for both of these elements has not been highlighted much in previous work, however, the larger number of abundances measurements in the Hypatia Catalog allows a better comparison of the trends. Due to the difficulty of measuring $[\mathrm{Zn} / \mathrm{Fe}]$, there were not enough stars to accurately determine the $1 \sigma$ trend lines about the median, which emphasizes the need to better understand the trends in Fig. 25 .

A total of 64 stars within the Hypatia Catalog have copper and zinc abundances determined by Ramírez et al. (2009). Their spectra has a resolution of $\lambda / \Delta \lambda \approx 60000$ over the range 3800-9125 $\AA$ and high signal-to-noise $(\mathrm{S} / \mathrm{N} \approx 200$ per spectral pixel) for the solar twins and analog stars. They determined the solar flux spectrum by using differential analysis from asteroid spectra. Effective temperatures, surface gravities, and microturbulent velocities were obtained by iterating until the difference in $[\mathrm{Fe} / \mathrm{H}]$ values from both $\mathrm{Fe}$ I and Fe II approached zero. Model LTE atmospheres from Kurucz (2005) and MOOG (Sneden 1973) were used to determine all element abundances. The reported uncertainties for the abundance measurements is $[\mathrm{X} / \mathrm{Fe}] \approx 0.03 \mathrm{dex}$.

There are multiple processes that produce strontium, yttrium, and zirconium. According to Arlandini et al. (1999), 85\% of strontium in the solar composition is from the r-process and $15 \%$ from the s-process. Similarly, $92 \%$ of yttrium and $83 \%$ of zirconium is through the r-process while $8 \%$ and $17 \%$, respectively, is from the s-process. While the relative contributions from each process depend on the initial enrichment and the age of the stellar system, there are unaccounted contributions at low metallicities (Thielemann et al. 2007). These abundance remainders might 

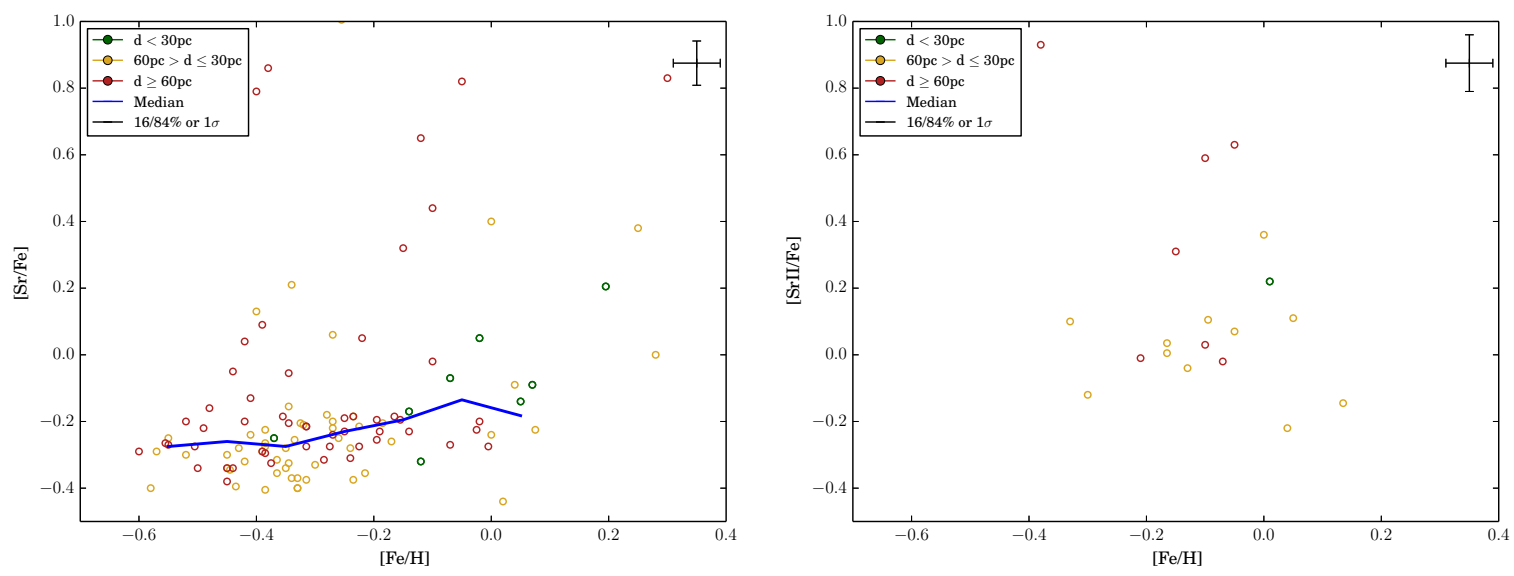

Fig. 26. - Same as Fig. 5 but for neutral (left) and ionized (right) strontium. Due to small number statistics, the median and/or percentile trend lines could not be accurately determined for $[\mathrm{Sr} / \mathrm{Fe}]$ and $[\mathrm{SrII} / \mathrm{Fe}]$, which both have less than 250 stellar measurements (see Table 5 ).

be due to primary production of strontium, yttrium, and zirconium within massive stars, possibly through neutrino-proton interactions (Arnould et al. 2007).

Nine literature sources within Hypatia measured strontium for 274 stars: Galeev et al. (2004); Luck \& Heiter (2005); Mashonkina et al. (2007); Reddy et al. (2003); Thevenin (1998). Due to the limited number of available lines in the optical spectrum, a number of these literature sources quoted high uncertainties from blended, weak lines. The abundance of $[\mathrm{Sr} / \mathrm{Fe}]$ as a function of $[\mathrm{Fe} / \mathrm{H}]$ is shown in Fig. 26 (left). The median of the $[\mathrm{Sr} / \mathrm{Fe}]$ abundances is plotted in blue with respect to $[\mathrm{Fe} / \mathrm{H}]$. Due to the small number of stars, the $1 \sigma$ regression lines could not be calculated, but the scatter appears relatively large, which may be indicative of multiple origin sites (Lai et al. 2007). The outlier stars, with $[\mathrm{Sr} / \mathrm{Fe}]>0.4$, are a product of a number of different catalogs, such as Allen \& Porto de Mello (2011); Thevenin (1998); Luck \& Heiter (2005). There are 25 stars in the analysis of the Hypatia Catalog, see $\$ 4$, which have strontium abundances determined from Sr II, as shown in in Fig. 26 (right). The scatter in [Sr II/Fe] is large with no clear clustering below solar, unlike $[\mathrm{Sr} / \mathrm{Fe}]$. No trend lines could be determined for $[\mathrm{SrII} / \mathrm{Fe}]$.

Many catalogs within Hypatia made the distinction between measuring neutral or singly ionized yttrium, although only Feltzing \& Gustafsson (1998) measured both Y I and Y II. Evolution of $[\mathrm{Y} / \mathrm{Fe}]$ as a function of $[\mathrm{Fe} / \mathrm{H}]$ for 191 stars is shown in Fig. 27 (left) while [Y II /Fe] versus [Fe/H] for 210 stars is shown on the right. The more numerous Y II measurements are due to rather weak and blended lines available for Y I in the optical spectrum. The median $[\mathrm{Y} / \mathrm{Fe}]$ abundance trend, represented by the blue line, suggests a flat and slightly sub-solar trend Qian \& Wasserburg 2008; Roederer et al. 2010a), but with a relatively large dispersion for most of $[\mathrm{Fe} / \mathrm{H}]$. The stars with super-solar $[\mathrm{Y} / \mathrm{Fe}]$, or greater than $0.5 \mathrm{dex}$, were measured by a number of catalogs, such as 

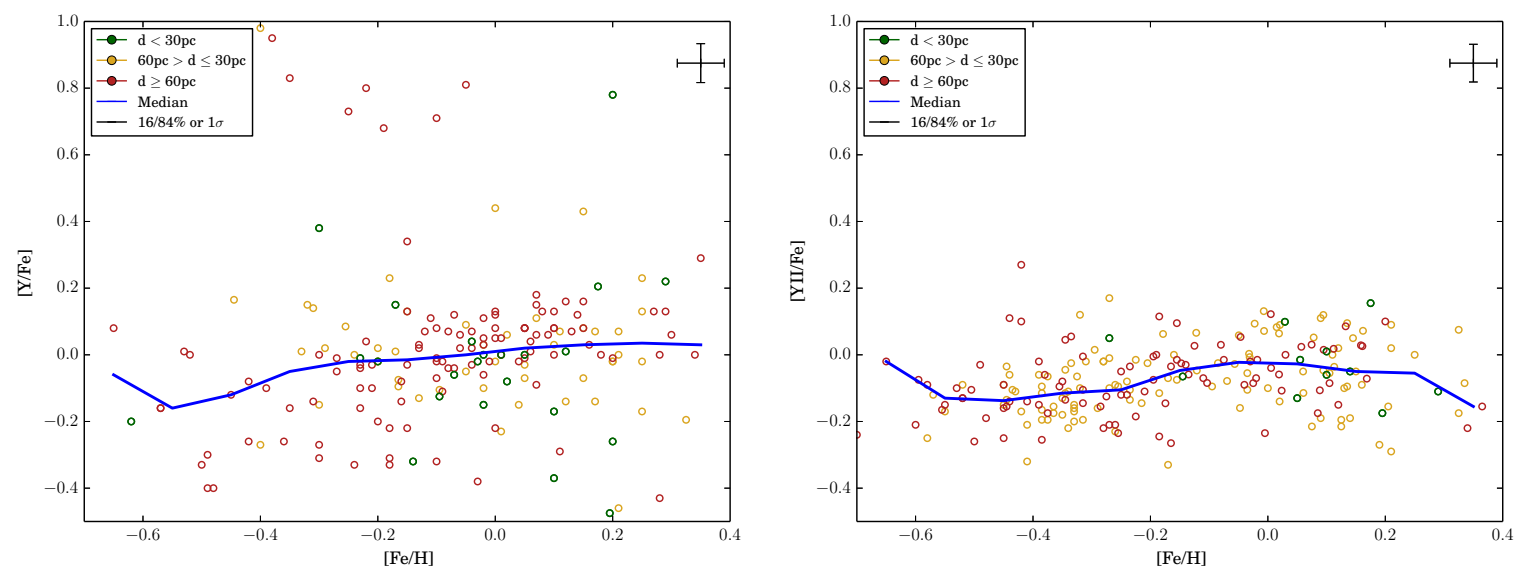

Fig. 27. - Same as Fig. 5 but for neutral (left) and ionized (right) yttrium. Due to small number statistics, the percentile trend lines could not be accurately determined for $[\mathrm{Y} / \mathrm{Fe}]$ and $[\mathrm{YII} / \mathrm{Fe}]$, which have less than 250 stellar measurements (see Table 5).

Thevenin (1998); Wang et al. (2011); Allen \& Porto de Mello (2011). Wang et al. (2011) used a mixture of both Y I and Y II lines in their abundance determinations, while the abundances in Allen \& Porto de Mello (2011) employed differing measurement techniques between lines (see their Table 2). The line list used by Thevenin (1998) was not published. Due to the abnormality of these super-solar $[\mathrm{Y} / \mathrm{Fe}]$ abundances, these results may be due to varying reduction methods. Until further investigation, the validity of their results remains tentative. There are relatively few stars with a radial distance less than $30 \mathrm{pc}$ and the majority of those stars exhibit near-solar or below $[\mathrm{Y} / \mathrm{Fe}]$ abundances. Comparatively, the evolution the $[\mathrm{Y} \mathrm{II} / \mathrm{Fe}]$ ratio with $[\mathrm{Fe} / \mathrm{H}]$ is also relatively flat and below-solar. However, the dispersion is much less than $[\mathrm{Y} / \mathrm{Fe}]$, most likely due to the smaller number of unblended Y II lines. This suggests that the smaller scatter in the [YII/Fe] measurements are more likely to be physical.

Zirconium abundances in Hypatia are similar to those of yttrium, in that the singly ionized state was preferentially measured due to blending of weak neutral lines in the optical spectrum. Evolution of $[\mathrm{Zr} / \mathrm{Fe}]$ as a function of $[\mathrm{Fe} / \mathrm{H}]$ for the 96 stars in the analysis of Hypatia (see 84 ) is shown in Fig. 28 (left), and [Zr II /Fe] versus [Fe/H] for 188 stars is given in Fig. 28 (right). Because both Zr and Zr II have been measured than less than 250 stars, their $1 \sigma$ quantile regression trends couldn't be determined. The abundances for $[\mathrm{Zr} / \mathrm{Fe}]$ are generally solar (Qian \& Wasserburg 2008, Kashiv et al. 2010), although with significant scatter that makes the trend difficult to discern. There are 14 stars with $[\mathrm{Zr} / \mathrm{Fe}]>0.4$ dex, contributed from Thevenin (1998); Allen \& Porto de Mello (2011). As discussed above, the varying techniques employed by within these datasets may have contributed to the widely varying $[\mathrm{Zr} / \mathrm{Fe}]$ measurements. There are few abundance measurements around $[\mathrm{Fe} / \mathrm{H}]<-0.4$ dex, as noticed by the sharp peak in the median line. Comparatively $[\mathrm{Zr}$ II/Fe] shows a shallow concave-up trend and less scatter, since the two plots in Fig. 28 have the 
same axes. The outlier stars, with $[\mathrm{ZrII} / \mathrm{Fe}]>0.25$ originate from both Reddy et al. (2003); Allen \& Porto de Mello (2011). Similarities between the patterns seen in [Zr II/Fe] and [Y II/Fe] with respect to $[\mathrm{Fe} / \mathrm{H}]$ suggest similar origin sites.
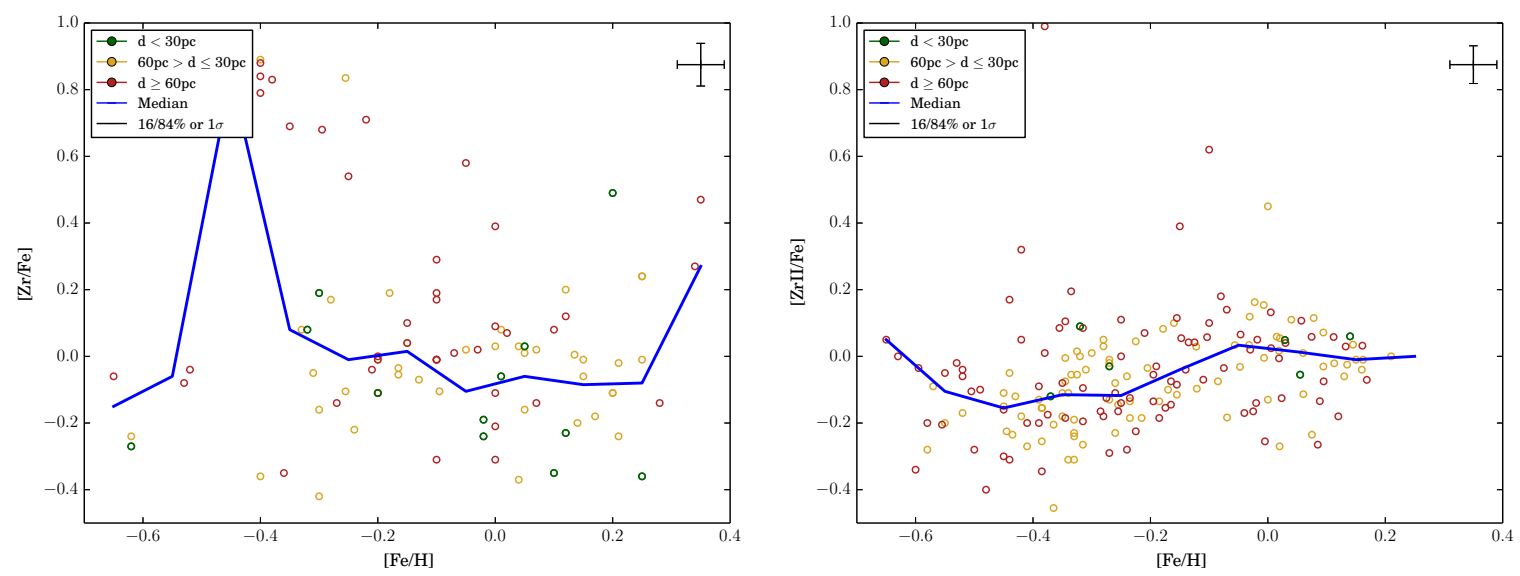

Fig. 28. - Same as Fig. 5 but for neutral (left) and ionized (right) zirconium. Due to small number statistics, the percentile trend lines could not be accurately determined for [ $\mathrm{Zr} / \mathrm{Fe}]$ and $[\mathrm{ZrII} / \mathrm{Fe}]$, which have less than 250 stellar measurements (see Table 5).

Bond et al. (2006) determined Y II and Zr II abundances in of 144 G-type stars, all of which are in Hypatia, using high resolution $(\lambda / \Delta \lambda \approx 80000)$, high signal-to-noise ratio ( $\mathrm{S} / \mathrm{N} \approx 250$ per pixel) spectra of the $5380.32 \AA$ and $6587.62 \AA$ lines of $\mathrm{C}$ I. Oscillator strengths and excitation energies for each line were obtained from the NIST Atomic Spectra Database. Effective temperatures were determined from the stellar colors listed in the Hipparcos Catalogue, which is different from similar studies that utilize the spectra for an effective temperature value, and resulted in a mean $T_{\text {eff }}$ uncertainty of $\pm 100 \mathrm{~K}$. An LTE analysis using WIDTH6 (Kurucz 2005) in conjunction with a grid of Kurucz (2005) ATLAS9 atmospheres was used to determine the elemental abundances. Surface gravities $\log g$ values and Fe abundances were obtained by iterating until the $[\mathrm{Fe} / \mathrm{H}]$ value from both Fe I and Fe II was the same. The microturbulence parameter $\zeta$ which minimized the correlation coefficient between $\log \zeta$ for Fe I and $\log \left(W_{\lambda} / \lambda\right)$ was selected with an estimated uncertainty of \pm 0.25 .

\section{Neutron-Capture Elements (Ru, Ba, La, Ce, Pr, Nd, Sm, Eu, Gd, Dy, \& Pb)}

The heavy neutron capture elements have the majority of their lines in the extreme blue and UV part of the spectrum, making it challenging to measure these elements (Spite \& Spite 1978; Sneden et al. 2008). As a result, fewer catalogs report these abundances (see Fig. 1) and the observational 
uncertainties in the abundance ratios are generally larger for the neutron capture elements. Because of the larger scatter seen in the data, the plots shown for the neutron-capture elements have $\mathrm{x}$ and $\mathrm{y}$-axis scales where that $[\mathrm{Fe} / \mathrm{H}]=[-0.7,0.4]$ and $[\mathrm{X} / \mathrm{Fe}]=[-0.6,1.0]$, respectively. We elect not to discuss $\mathrm{Ru}, \mathrm{La}, \mathrm{Pr}, \mathrm{Sm}, \mathrm{Gd}$, Dy and $\mathrm{Pb}$ in this paper, although these elements are listed in Hypatia, focusing on those elements with more measurements: Ba II, Ce, Nd, and Eu.

Elemental barium is dominated by three isotopes: ${ }^{135} \mathrm{Ba},{ }^{137} \mathrm{Ba}$, and ${ }^{138} \mathrm{Ba}$, the majority of which are made in the s-process (Arlandini et al. 1999; Travaglio et al. 1999; Mashonkina \& Gehren 2000; Carlson et al. 2007). Only singly ionized barium lines are available in the optical band for FGK-type stars, which are strongly affected by hyperfine splitting (Mashonkina \& Gehren 2000, Mashonkina et al. 2007). Variation of [Ba II/Fe] with $[\mathrm{Fe} / \mathrm{H}]$ for the 301 stars in the analysis of Hypatia is shown in Fig. 29 (left). The median and $1 \sigma$ regression lines suggest a relatively flat and slightly sub-solar [Ba II/Fe] ratio with a large scatter of $\approx 0.26$ dex (see Table 4). There are also a number of outlier stars with extremely enriched Ba II at distances greater than $30 \mathrm{pc}$, some of which were determined by Allen \& Porto de Mello (2011). Similar to yttrium and zirconium, the stars that are closer to the Sun (green circles) have higher $[\mathrm{Fe} / \mathrm{H}]$ content as well as near- or below-solar values of $[\mathrm{Ba} \mathrm{II} / \mathrm{Fe}]$.
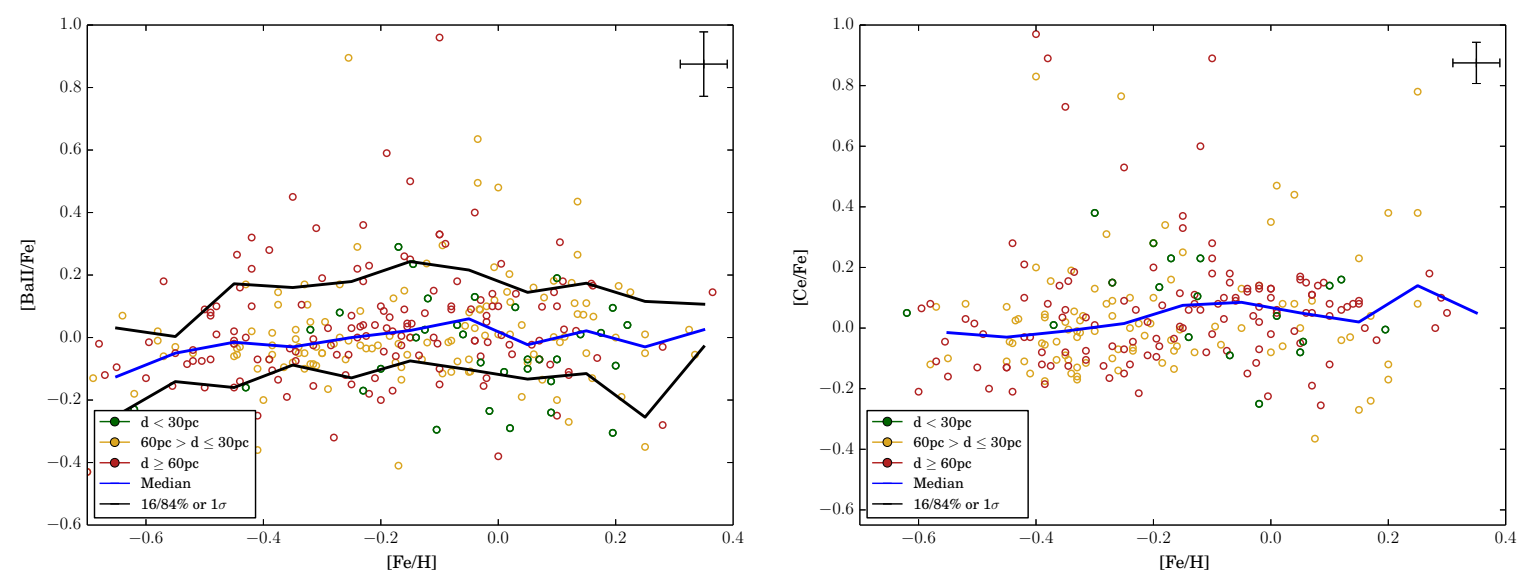

Fig. 29. - Same as Fig. 5 but for ionized barium (left) and cerium (right). Due to small number statistics, the percentile trend lines could not be accurately determined for [Ce/Fe], which has less than 250 stellar measurements (see Table 5).

Cerium is also predominantly $(77 \%)$ made by made by the s-process as either ${ }^{140} \mathrm{Ce}$ or ${ }^{142} \mathrm{Ce}$ Arlandini et al. (1999); Simmerer et al. (2004); Käppeler et al. (2011). Twelve catalogs within Hypatia measured cerium in 237 stars, due to only one or two lines in the optical spectrum that were not strongly affected by blending (Luck \& Heiter 2005). Fig. 29 (right) shows a predominantly near-solar $[\mathrm{Ce} / \mathrm{Fe}]$ trend with respect to $[\mathrm{Fe} / \mathrm{H}]$, as highlighted by the median curve, also noted by Brewer \& Carney (2006). Given the small number of stars, the scatter was difficult to determine using the $1 \sigma$ quantile regression analysis. However, similar to $[\mathrm{BaII} / \mathrm{H}]$, Allen \& Porto de Mello 
(2011), as well as Thevenin (1998), reported multiple extremely high [Ce/Fe] abundance ratios for the more distant stars.

Determinations for a wide-variety of neutron-capture elements were conducted by Galeev et al. (2004), who measured Ru, Ba II, La, Ce, Pr, Nd, Sm, and Eu abundances for 15 stars in the solar neighborhood, all of which are in the Hypatia Catalog, using the $2 \mathrm{~m}$ reflector at Terskol Observatory in the northern Caucasus. Their high resolution spectra $(\lambda / \Delta \lambda \approx 45000)$ covered $4000-9000 \AA$ with a signal-to-noise ratio of 150-200 per spectral pixel. They determined the solar flux spectrum via differential analysis, using solar light scattered off of the Earth's atmosphere. An LTE analysis with WIDTH6 (Kurucz 2005) is used, in conjunction with oscillator strengths from the VALD database (Kupka et al. 1999), to determine the final elemental abundances. An accuracy of 0.10 dex is assigned to their $[\mathrm{Fe} / \mathrm{H}]$ measurements.

The seven stable isotopes of neodymium are made by both the $\mathrm{r}$ - and the s-processes (Roederer et al. 2008). Due to blending with nearby lines, there are only one or two lines in the optical spectrum from which $[\mathrm{Nd} / \mathrm{Fe}]$ is measured (Galeev et al. 2004; Bond et al. 2008). The median $[\mathrm{Nd} / \mathrm{Fe}]$ abundance, shown by the blue line, indicates a relatively flat, solar trend for $[\mathrm{Nd} / \mathrm{Fe}]$ with $[\mathrm{Fe} / \mathrm{H}]$, see Table 4 as well as Thevenin (1998); Reddy et al. (2003). Due to the small number of stars for which this element was measured, as well as the large scatter (which was not due to directly to any one catalog), it is difficult to discern any robust trend.
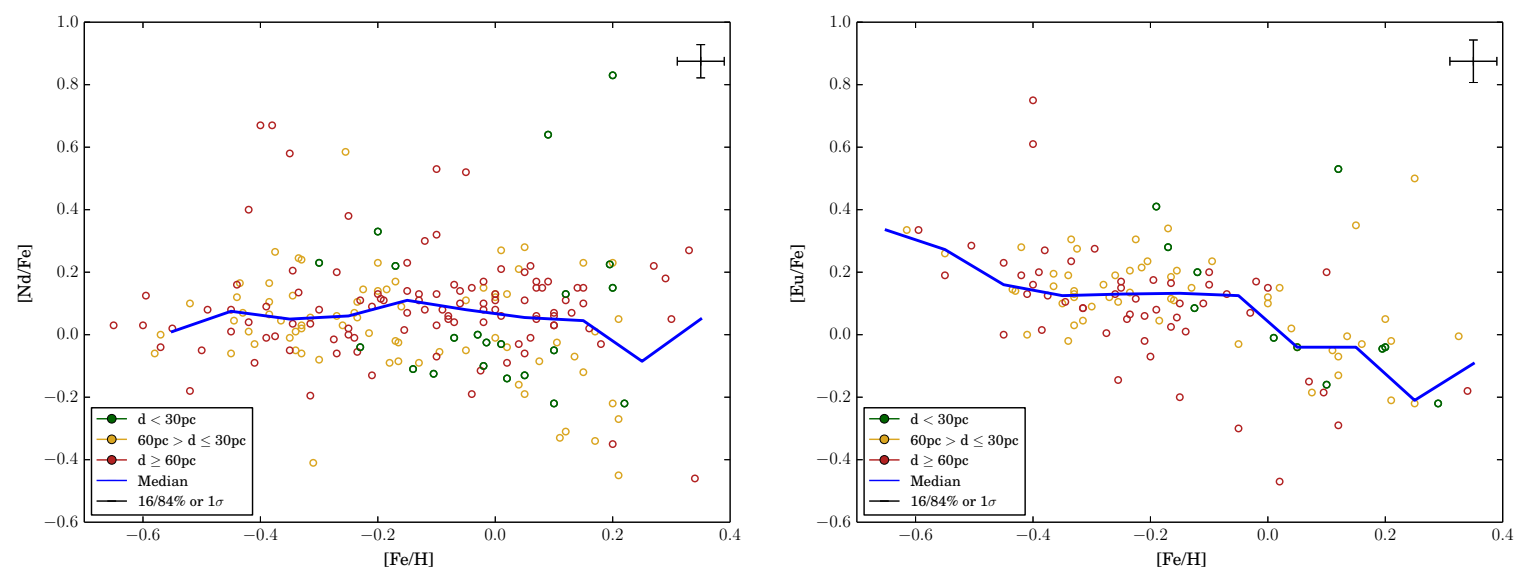

Fig. 30.- Same as Fig. 5 but for neodymium (left) and europium (right). Due to small number statistics, the percentile trend lines could not be accurately determined for $[\mathrm{Nd} / \mathrm{Fe}]$ and $[\mathrm{Eu} / \mathrm{Fe}]$, which have less than 250 stellar measurements (see Table 5).

About $91 \%$ of europium is estimated to be from the r-process (Cameron 1982; Arlandini et al. 1999) and is often used as a standard against a predominantly s-process element, such as [Ba/Eu] (McWilliam 1997; Travaglio et al. 1999; Mashonkina \& Gehren 2000). There are eighteen literature sources within Hypatia that measured $[\mathrm{Eu} / \mathrm{Fe}]$ using one or two lines in the optical spectrum. 
Unlike neodymium, the trend of $[\mathrm{Eu} / \mathrm{Fe}]$ with respect to $[\mathrm{Fe} / \mathrm{H}]$ has a negative slope with $[\mathrm{Fe} / \mathrm{H}]$ (Thevenin 1998; Reddy et al. 2003; Galeev et al. 2004; Bond et al. 2008; Allen \& Porto de Mello 2011), as shown in Fig. 30 (right). However, the overall scatter away from the median trend line is relatively large, $\approx 0.8$ dex over the range of $[\mathrm{Fe} / \mathrm{H}]$ shown. There were few $[\mathrm{Eu} / \mathrm{Fe}]$ measurements for stars at distances near to the Sun. In general, the abundance measurements (most of which were from stars greater than $30 \mathrm{pc}$ ) tend to be clustered at $[\mathrm{Eu} / \mathrm{Fe}] \approx 0.0 \operatorname{dex}$ and $[\mathrm{Fe} / \mathrm{H}] \approx-0.2$ $\operatorname{dex}($ Thevenin 1998; Allen \& Porto de Mello 2011).

\section{Gaps in Abundances}

We found a number of elements, specifically Mg, Si, S, Sc II, Cr II, and Ni, that displayed systematic correlations, namely two ensembles separated by a "gap" in their abundance ratios relative to $\mathrm{Fe}$. In each case, the ensemble with a higher $[\mathrm{X} / \mathrm{Fe}]$ tends to be at lower $[\mathrm{Fe} / \mathrm{H}]$, though there is overlap. In all cases, the ensembles showed a bias with respect to stellar distance. Stars below the gap are closer, on average than the stars above. In some cases the majority of the stars contributing to the lower ensemble were attributable to a single survey. However, the dominant survey was different in the cases of different elements (i.e. Neves et al. (2009) for Mg, Valenti \& Fischer (2005) for $\mathrm{Si}$ ). Furthermore, when the survey was removed, the distinct ensembles were still identifiable, but with smaller statistics. These were often stars with abundances measured in multiple surveys. Further investigation revealed that the surveys that contributed large numbers of stars to the lower ensemble, for example Neves et al. (2009); Valenti \& Fischer (2005), analyzed more nearby stars. That being said, the accumulation of a number of datasets that reveal this trend, including those listed in each respective subsection, for example Sadakane et al. (2002); Neves et al. (2009), leads us to believe that what we are seeing is two physically distinct groups of stars. We did not include $\mathrm{Ca}$ and $\mathrm{Ti}$ in this list as they do not have the immediately obvious gap shown by the elements listed above. We do, however, examine their statistics, since they are products of $\alpha$-chain burning like $\mathrm{Mg}, \mathrm{Si}, \mathrm{S}$, and $\mathrm{Cr}$. Ca does show a less prominent gap. There is only a bare suggestion of a gap in Ti, but the stair-step morphology of the plot at $0.0<[\mathrm{Fe} / \mathrm{H}]<$ 0.2 is similar to the other elements considered. In both cases the division is close to $[\mathrm{X} / \mathrm{Fe}]=-0.1$, as with the other elements.

One simple demonstration of the reality of the "gaps" is the direct analysis of two stellar spectra, with roughly equally $[\mathrm{Fe} / \mathrm{H}]$ but differing elemental abundances. In Figure 31 , we show spectra from HD 109591(blue) and HD 50255 (pink). To the left and middle, we present two Fe I lines, where the percent difference between the equivalent width determinations per Pagano (2014) is $0.069 \%$ and $1.6 \%$, respectively. For the three Ni I lines on the right, the difference between the equivalent widths are $7.5 \%, 10.4 \%$, and $11.8 \%$, respectively. Pagano (2014) also derived the stellar parameters and abundances for these stars: $T_{\text {eff }}=5784,5788 \mathrm{~K} ;[\mathrm{Fe} / \mathrm{H}]=-0.02,-0.04$ dex; and $[\mathrm{Ni} / \mathrm{Fe}]=0.01,-0.14 \mathrm{dex}$, respectively. Notably, while the effective temperatures and overall metallicity are the same, the nickel content (both in the raw spectra and reduced abundance ratio) 
vary dramatically between the two stars.
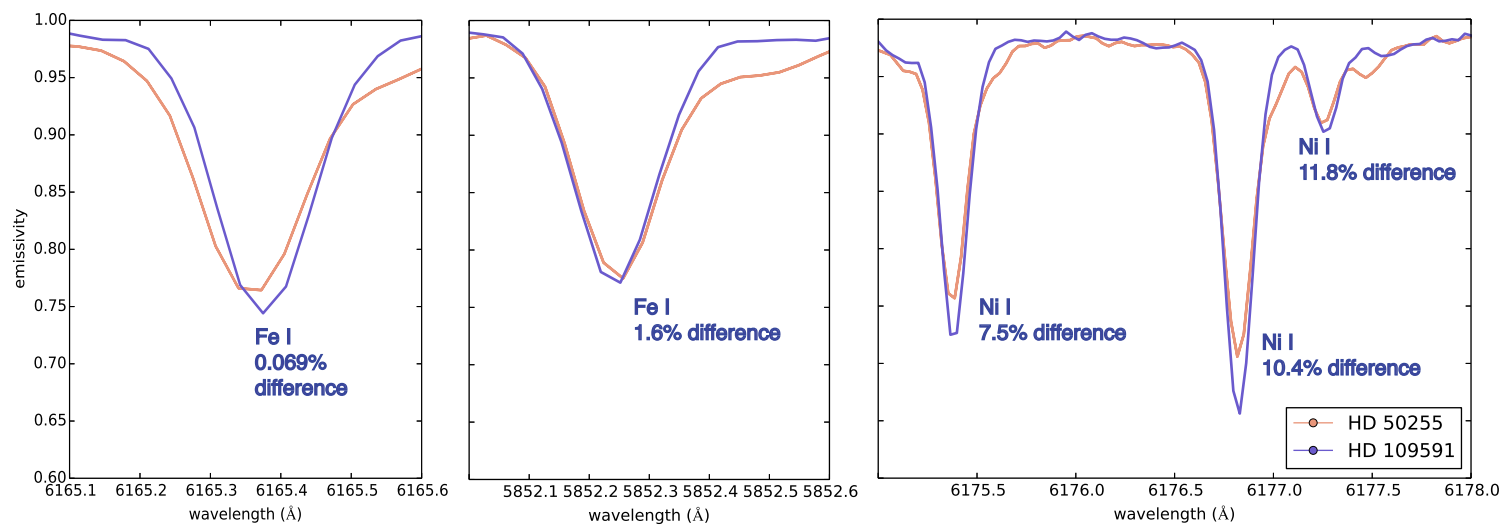

Fig. 31.- Continuum normalized spectra for two stars with nearly equal iron abundance, see lines on the left and middle, but noticeably differing nickel abundances, see three lines on the right (Pagano 2014).

To better understand the stars within each ensemble above and below the gap, we analyzed the z-height distribution with respect to the Galactic plane. In Fig. 32, we show the stars with silicon measurements (left) and nickel abundances (right), with exponential fits to the distributions. There is overlap between the ensembles, with both having members at all heights above the disk, but it is clear that the ensemble below the gap is more concentrated towards the mid-plane of the disk. Alternatively, we also examine the height $h_{90}$ below which $90 \%$ of the stars in each ensemble can be found. Table 6 gives the scale heights of the exponential fits $h_{a}$ and $h_{b}$ for the ensembles above and below the gap, respectively, along with $h_{90 a}$ and $h_{90 b}$. For the definitive gap elements, in all cases $h_{a}$ falls between 35 and 40pc. Excluding sulfur, $h_{b}$ shows a slightly larger range, from 21 to $28 \mathrm{pc}$. The scale height for sulfur below the gap is $34 \mathrm{pc}$, but there are fewer than 60 stars upon which to base the fit. The $90 \%$ limits show a similar pattern with a moderately larger range. Values for $h_{90 a}$ vary from 68 to $80 \mathrm{pc}$. Again excepting sulfur, $h_{90 b}$ ranges from 36 to $50 \mathrm{pc}$. The below the gap ensemble for $\mathrm{S}$ is closer to but still lower than the ensemble above the gap. The cases of $\mathrm{Si}$ and $\mathrm{Ni}$, shown in Figure 32, actually show the least difference between the vertical distributions of the ensembles if $\mathrm{S}$ is removed. In both cases $h_{b}$ is $70 \%$ of $h_{a}$, and $h_{90 b} 61 \%$ and $63 \%$ of $h_{90 a}$, respectively. The addition of the potential gap elements changes the picture very little. Calcium is solidly within the normal distribution for both $h$ and $h_{90}$. The separation in Ti is relatively small, with $h_{a}$ and $h_{b}$ differing by $3 \mathrm{pc}$, though there is still a difference of $21 \mathrm{pc}$ in $h_{90}$.

For an additional test regarding the contribution of individual datasets to Hypatia, we recalculated the scale-heights without the inclusion of the Neves et al. (2009) dataset, one of the largest contributors for many of the lower "ensembles." We then recalculated the scale-heights to better understand the impact of their survey, see Table 7. We found that the scale-heights did not vary more than $2 \mathrm{pc}$ for either ensemble in $\mathrm{Mg}, \mathrm{Si}, \mathrm{S}$, and $\mathrm{Ni}$, or for the ensemble above the gap in $\mathrm{Ca}$ 

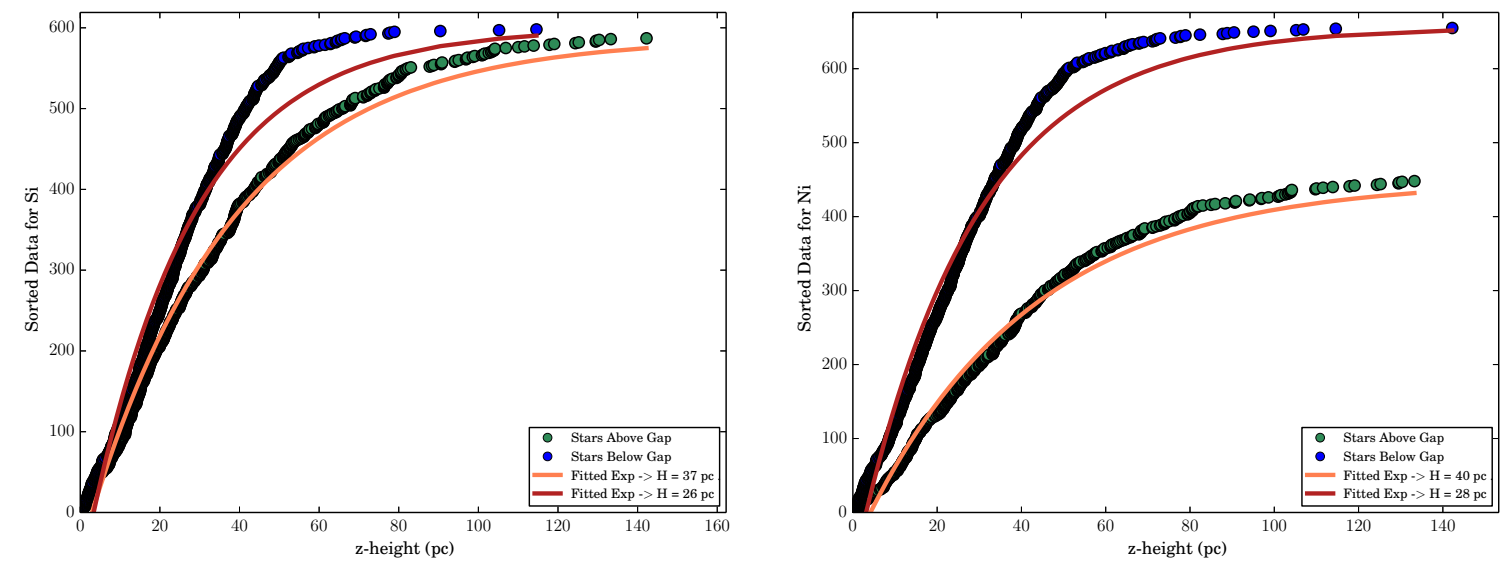

Fig. 32.- Sorted z-height data above and below the respective gaps for stars with silicon (left) and nickel (right) abundances. The legend gives the scale height $(\mathrm{H})$ as a result of the fitted exponentials.

and Cr. The $h_{a}$ for Sc dropped by 5pc, with roughly half the number of stars as the full sample. The $h_{a}$ for $\mathrm{Ti}$ increased by $4 \mathrm{pc}$, actually making the ensembles above and below more distinct. The value of $h_{b}$ changed by less than $2 \mathrm{pc}$ for all elements except Ca and Cr. In both cases there were fewer than 20 stars remaining below the gap when the survey was removed. The situation was similar for $h_{90}$. In every case but two (6pc for Sc and $4 \mathrm{pc}$ for $\left.\mathrm{Ti}\right) h_{90 a}$ changed by $2 \mathrm{pc}$ or less. The value of $h_{90 b}$ changed by no mare than $2 \mathrm{pc}$ for all of the elements except $\mathrm{Ca}, \mathrm{Sc}$, and $\mathrm{Cr}$. In all of these cases the number of stars remaining below the gap was $\lesssim 20$. We would expect this measure to be very sensitive to small number statistics. In general, we found that the structure of the two "ensembles" is unaffected by an individual catalog.

This set of tests shows remarkable consistency, and suggests that the stars above and below the gap may in fact be physically distinct ensembles. We find that there may be an inhomogeneity in the solar neighborhood stars, specifically a group near to the mid-plane that is high in $[\mathrm{Fe} / \mathrm{H}]$ but deficient in the elements $\mathrm{Mg}, \mathrm{Si}, \mathrm{S}, \mathrm{Ca}, \mathrm{Sc} \mathrm{II}, \mathrm{Ti}, \mathrm{Cr}$ II, and Ni. The case is particularly strong for $\mathrm{Mg}, \mathrm{Si}, \mathrm{S}, \mathrm{Ca}, \mathrm{Sc}, \mathrm{Cr}$, and Ni. Titanium shows a similar overall morphology, though the gap is at least partially filled. The others, $\mathrm{Mg}, \mathrm{Si}, \mathrm{S}$, and $\mathrm{Ca}$, are $\alpha$-elements, and the dominant isotopes of $\mathrm{Ti}$ and $\mathrm{Cr}$ are direct products of $\alpha$-chain burning $\left({ }^{48} \mathrm{Ti}\right.$ and ${ }^{52} \mathrm{Cr}$ are beta decay products of ${ }^{48} \mathrm{Cr}$ and ${ }^{52} \mathrm{Fe}$, respectively.) Nickel is a primarily product of $\alpha$-captures onto ${ }^{54} \mathrm{Fe}$ and ${ }^{56} \mathrm{Fe}$ in a moderately neutron rich environment. Scandium is an odd-z element produced mostly in corecollapse SNe. It would be instructive to look for similar behavior in the nearby odd-z elements $\mathrm{V}$ and $\mathrm{Mn}$, but these have extremely high spreads, perhaps due to incorrect handling of hyperfine corrections as noted above. Contributions from SNIa may be partly obscuring the gap in Ti in particular, and to an extent in Ca. We note that the gap occurs most clearly for elements that are dominated by a single nucleosynthetic production site, explosive $\mathrm{O} / \mathrm{Si}$ burning or $\alpha$-rich freezeout 
in core-collapse SNe. Other nucleosynthetic sources or observational vagaries may be obscuring a gap that would otherwise appear for other elements. It will prove valuable to explore this possible nearby asymmetric abundance distribution, particularly as it indicates that the solar neighborhood is not well-mixed compositionally. Information on ages and detailed kinematics of these ensembles could prove highly interesting.

\section{Discussion}

We have assembled spectroscopic abundance data from 84 literature sources for 50 elements across 3058 stars within 150 pc of the Sun to build the Hypatia Catalog. This is the largest, most complete catalog of spectroscopic abundance data for stars in the solar neighborhood to date, of which we are aware (see Fig. 1). The aim of this research is to (a) provide an unbiased compilation of abundance determinations of main-sequence stars in the solar neighborhood; (b) show the zeroth order abundance trends within Hypatia are consistent with the published literature; and (c) explicitly show the challenges of combining disparate data sets in hopes for some reconciliation within the community.

We encountered a number of issues by trying to amalgamate such large and diverse data sets, which we attempted to ameliorate in the most unbiased manner. We began by first determining the likely origin for the stars within the Hypatia Catalog using the prescription in Bensby et al. (2003), in order to exclude thick-disk stars from our analysis. We then undertook to minimize the spread, or the variation in abundance measurements for the same star and element reported by different literature sources (see Fig. 3), and renormalizing all the stars in Hypatia to the Lodders et al. (2009) solar abundance scale. We found that while this has a significant impact to the abundances (0.6 dex on average), it did not significantly affect the spread in the data. Finally, we only retained those stars in our analysis whose spread in both $[\mathrm{X} / \mathrm{Fe}]$ and $[\mathrm{Fe} / \mathrm{H}]$ was less than the respective error bar. In this way, we were able to ensure that the abundances we studied were agreed upon by multiple datasets, were well understood, and were without controversy (\$4).

We found abundance trends that are consistent with previously discovered average trends. In addition, the large number of stars in Hypatia allows us to observationally quantify the extent of the scatter, or the width about the median abundance trend using quantile regression for the $16 \%$ and $84 \%$ (total $68 \%$ or 1 standard deviation) percentiles, for each element (Figs. 5 . 30 , and also see Tinsley 1980; Malinie et al. 1993; van den Hoek \& de Jong 1997; Kobayashi \& Nakasato 2011). As a result of our careful analysis, we find that the solar neighborhood may not be homogeneously wellmixed. A number of elements revealed two distinct "ensembles" of stars with a possible asymmetric abundance distribution. Stars that were consistently near to the Galatic mid-plane were deficient

in $\mathrm{Mg}, \mathrm{Si}, \mathrm{S}, \mathrm{Ca}, \mathrm{Sc}$ II, Cr II, and $\mathrm{Ni}$ but enriched in $\mathrm{Fe}$, as opposed to stars further from the mid-plane, which implies a possible variation in the nearby chemical history.

One of the problems, when analyzing the abundance trends in the majority of literature sources, 
is that few data sets have enough stars to discern obvious patterns that are statistically viable. The Hypatia Catalog contains a large amount of both kinematic and abundance data. Some applications of this data include stellar abundance trends between thin and thick disk stars, slow and fast rotating stars, stars of different spectral types (or effective temperatures), exoplanet hosts versus stars confirmed to be without exoplanets, or solar analog stars. Hypatia may also be used to supplement pre-existing surveys such as NASA's Transiting Exoplanet Survey Satellite (TESS) mission, the ESA/NASA Herschel mission, or the Sloan Digital Sky Survey. None of the methods that we undertook in our analysis are reflected in the published version of the Hypatia Catalog, which contains only the data as determined by the original authors. In this way, others may analyze whatever datasets they choose in the way they see fit, making Hypatia an excellent resource. The breadth of information present within the Hypatia Catalog also makes it useful for gleaning new stellar information, such as stellar ages and the kinematics of the solar neighborhood.

\section{Acknowledgments}

The authors thank John Shumway, Scott Ransom, Sandra Schmidt, Matt Mechtley, Mark Richardson, Themis Athanassiadou, Jade Bond, Chris Sneden, and Caleb Wheeler for helpful discussions and computer support. The authors are grateful for the VIZIER, ADS, and SIMBAD databases. This work is supported by the NASA Astrobiology Institute under Grant 08-NAI5-0018 "Follow the Elements," and by the NSF under Grant PHY 02-16783 for the Frontier Center "Joint Institute for Nuclear Astrophysics". Turnbull thanks Ariel Anbar for the opportunity to join the NASA NAI "Follow the Elements" team. 
Table 1: Example of the Full Hypatia Catalog

$$
\begin{aligned}
& \text { Star: HIP }=400 \\
& \mathrm{HD}=225261 \\
& \mathrm{BD}=\mathrm{B}+224950 \\
& \text { Spec Type }=\mathrm{G} 9 \mathrm{~V} \\
& \text { dist }(\mathrm{pc})=26.39 \\
& \text { RA/Dec }=(1.23,23.27) \\
& \text { Disk component: thin } \\
& \text { NaH }-0.31 \text { [Valenti \& Fischer }(2005)] \\
& \mathrm{SiH}-0.23 \text { [Valenti \& Fischer }(2005)] \\
& \text { TiH }-0.22 \text { [Valenti \& Fischer }(2005)] \\
& \text { FeH }-0.23 \text { [Valenti \& Fischer }(2005)] \\
& \text { NiH -0.4 [Valenti \& Fischer }(2005)] \\
& \text { OH 0.02 [Petigura \& Marcy }(2011)] \\
& \text { FeH -0.23 [Petigura \& Marcy }(2011)]
\end{aligned}
$$




\begin{tabular}{|c|c|c|}
\hline Literature Reference & Stars & Elements \\
\hline Allen \& Porto de Mello (2011) & 33 & $(\mathrm{Fe}, \mathrm{Mn}, \mathrm{Cu}, \mathrm{Zn}, \mathrm{Y}, \mathrm{Ba}$ II, Nd, Eu, Gd, Dy) \\
\hline Allende Prieto et al. (2004) & 118 & (C, O, Mg, Si, Ca II, Sc II, Ti II, Fe, Co, Ni, Cu, Zn, Y, Ba II, Ce, Nd, Eu) \\
\hline Bensby et al. (2005) & 144 & (Fe, Na, Mg, Al, Si, Ca, Ti, Cr, Ni, Zn, Y II, Ba II) \\
\hline Bergemann \& Cescutti (2010) & 8 & (Fe, Cr, Cr II, Mg) \\
\hline Bodaghee et al. (2003) & 120 & (Fe, Si, Ca, Sc II, Ti, V, Cr, Mn, Co, Ni) \\
\hline Boesgaard et al. (2011) & 52 & $(\mathrm{Fe}, \mathrm{O}, \mathrm{Be}, \mathrm{Ti}, \mathrm{Mg})$ \\
\hline Bond et al. 2006 2008) & 144 & (Fe, C, Na, Al, Si, Ca, Ti, Ti II, Ni, O, Mg, Cr, Ba II, Y II, Zr II, Eu, Nd) \\
\hline Brewer \& Carney (2006) & 531 & $\begin{array}{l}(\mathrm{O}, \mathrm{Na}, \mathrm{Mg}, \mathrm{Al}, \mathrm{Si}, \mathrm{Ca}, \mathrm{Ti}, \mathrm{Sc}, \mathrm{V}, \mathrm{Cr}, \mathrm{Mn}, \mathrm{Fe}, \mathrm{Co}, \mathrm{Ni}, \mathrm{Cu}, \mathrm{Zn}, \mathrm{Sr}, \mathrm{Y}, \mathrm{Zr}, \mathrm{Ba} \\
\text { II, La, Ce, Nd, Eu) }\end{array}$ \\
\hline Brugamyer et al. (2011) & 121 & $(\mathrm{Fe}, \mathrm{Si}, \mathrm{O})$ \\
\hline Caffau et al. $(2011)$ & 20 & $(\mathrm{Fe}, \mathrm{P})$ \\
\hline Carretta et al. (2000) & 19 & $(\mathrm{Fe}, \mathrm{C}, \mathrm{N}, \mathrm{O}, \mathrm{Na}, \mathrm{Mg})$ \\
\hline Castro et al. (1997) & 4 & (Na, Mg, Si, Ca, Ti, V, Ni, Zr, Y, Ba II, Eu II, Fe) \\
\hline Castro et al. (1999) & 13 & $(\mathrm{Fe}, \mathrm{Cu}, \mathrm{Ba} \mathrm{II})$ \\
\hline Chen et al. $(2000)$ & 88 & $(\mathrm{Fe}, \mathrm{O}, \mathrm{Na}, \mathrm{Mg}, \mathrm{Al}, \mathrm{Si}, \mathrm{K}, \mathrm{Ca}, \mathrm{Ti}, \mathrm{V})$ \\
\hline da Silva et al. (2012) & 25 & $\begin{array}{l}\text { (Fe, C, Na, Mg, Si, Ca, Sc, Ti, V, Cr, Mn, Co, Ni, Cu, Zn, Sr, Y II, Zr, Ba } \\
\text { II, Ce II, Nd II, Sm II) }\end{array}$ \\
\hline Delgado Mena et al. (2010) & 369 & $(\mathrm{Fe}, \mathrm{O}, \mathrm{Ni}, \mathrm{C}, \mathrm{Mg}, \mathrm{Si})$ \\
\hline D'Orazi et al. $(2012)$ & 6 & (Fe, Y II, Zr II, Ba II, La II, Ce II) \\
\hline Ecuvillon et al. 2004 & 126 & $(\mathrm{Fe}, \mathrm{Zn}, \mathrm{Cu}, \mathrm{C}, \mathrm{S})$ \\
\hline Ecuvillon et al. (2006) & 93 & $(\mathrm{Fe}, \mathrm{O})$ \\
\hline Edvardsson et al. (1993) & 180 & (Fe, O, Na, Mg, Al, Si, Ca, Ti, Ni, Y II, Zr II, Ba II, Nd) \\
\hline Feltzing \& Gustafsson (1998) & 45 & $\begin{array}{l}\text { (O, Na, Mg, Al, Si, Ca, Sc, Sc II, Ti, V, V II, Cr, Cr II, Mn, Fe, Co, Ni, Y, } \\
\text { Y II, Zr, Mo, La, Nd, Eu, Hf) }\end{array}$ \\
\hline Feltzing et al. (2007) & 95 & $(\mathrm{Fe}, \mathrm{Mn})$ \\
\hline Francois 1986 & 36 & $(\mathrm{Fe}, \mathrm{Al}, \mathrm{Si}, \mathrm{Na}, \mathrm{Mg})$ \\
\hline Francois $(\overline{1988})$ & 11 & $(\mathrm{Fe}, \mathrm{S})$ \\
\hline Fulbright (2000) & 166 & (Fe, Na, Mg, Al, Si, Ca, Ti, V, Cr, Ni, Y II, Zr II, Ba II, Eu) \\
\hline Galeev et al. $(2004)$ & 15 & $\begin{array}{l}(\mathrm{Li}, \mathrm{C}, \mathrm{N}, \mathrm{O}, \mathrm{Na}, \mathrm{Mg}, \mathrm{Al}, \mathrm{Si}, \mathrm{S}, \mathrm{K}, \mathrm{Ca}, \mathrm{Sc}, \mathrm{Ti}, \mathrm{V}, \mathrm{Cr}, \mathrm{Mn}, \mathrm{Fe}, \mathrm{Co}, \mathrm{Ni}, \mathrm{Cu} \text {, } \\
\mathrm{Zn}, \mathrm{Sr}, \mathrm{Y}, \mathrm{Zr}, \mathrm{Mo}, \mathrm{Ru}, \mathrm{Ba} \mathrm{II}, \mathrm{La}, \mathrm{Ce}, \mathrm{Pr}, \mathrm{Nd}, \mathrm{Sm}, \mathrm{Eu})\end{array}$ \\
\hline Gebran et al. (2010) & 28 & (C, O, Na, Mg, Si, Ca II, Sc II, Fe, Ni, Y II) \\
\hline Gilli et al. (2006) & 98 & $(\mathrm{Si}, \mathrm{Ca}, \mathrm{Sc} \mathrm{II}, \mathrm{Ti}, \mathrm{V}, \mathrm{Cr}, \mathrm{Mn}, \mathrm{Co}, \mathrm{Ni}, \mathrm{Fe})$ \\
\hline Gonzalez et al. (2001) & 22 & (Fe, C, O, Na, Al, Si, Ca, Sc, Ti, Ni) \\
\hline Gonzalez \& Laws (2007) & 31 & (Fe, Li, C, N, Al, Ca, Mg, Na, S, Sc, Si, Ti, Cr, Cu, Mn, Ni, Zn, Eu) \\
\hline González Hernández et al. & 95 & $\begin{array}{l}\text { (Fe, C, O, S, Na, Mg, Al, Si, Ca, Sc, Ti, V, Cr, Mn, Co, Ni, Cu, Zn, Sr, Y, } \\
\mathrm{Zr}, \mathrm{Ba} \text { II, Ce, Nd, Eu) }\end{array}$ \\
\hline Gratton et al. 2000 & 58 & $(\mathrm{Fe}, \mathrm{C}, \mathrm{N}, \mathrm{O}, \mathrm{Na})$ \\
\hline Gratton et al. $(2003)$ & 116 & (Fe, O, Na, Mg, Si, Ca, Ti, Ti II, Sc II, V, Cr, Cr II, Mn, Ni, Zn) \\
\hline Gustafsson et al. (1999) & 80 & $(\mathrm{Fe}, \mathrm{C}, \mathrm{O})$ \\
\hline Huang et al. $(2005)$ & 22 & $(\mathrm{C}, \mathrm{O}, \mathrm{Na}, \mathrm{Mg}, \mathrm{Al}, \mathrm{Si}, \mathrm{S}, \mathrm{Ca}, \mathrm{Sc} \mathrm{II}, \mathrm{Ti}, \mathrm{V}, \mathrm{Cr}, \mathrm{Mn}, \mathrm{Ni}, \mathrm{Ba}$ II, Fe) \\
\hline Jonsell et al. $(2005)$ & 43 & (Fe, O, Na, Mg, Al, Si, Ca, Sc II, Ti, V, Cr, Ni, Ba II) \\
\hline Kang et al. $(2011)$ & 51 & (Fe, Na, Mg, Al, Si, Ca, Sc, Ti, T III, V, Cr, Mn, Co, Ni) \\
\hline Koch \& Edvardsson (2002) & 74 & $(\mathrm{Eu}, \mathrm{Fe})$ \\
\hline Korotin et al. (2011) & 172 & $(\mathrm{Fe}, \mathrm{Ba} \mathrm{II})$ \\
\hline Laird (1985) & 116 & $(\mathrm{Fe}, \mathrm{C}, \mathrm{N})$ \\
\hline Luck \& Heiter (2005) & 110 & $\begin{array}{l}\text { (C, O, Na, Mg, Al, Si, S, Ca, Sc, Ti, V, Cr, Mn, Fe, Co, Ni, Cu, } \\
\mathrm{Zn}, \mathrm{Sr}, \mathrm{Y}, \mathrm{Zr}, \mathrm{Ba} \mathrm{II}, \mathrm{La}, \mathrm{Ce}, \mathrm{Pr}, \mathrm{Nd}, \mathrm{Sm}, \mathrm{Eu})\end{array}$ \\
\hline Mashonkina \& Zhao (2006) & 25 & $(\mathrm{Fe}, \mathrm{Ba} \mathrm{II})$ \\
\hline Mashonkina et al. $(2007)$ & 67 & $(\mathrm{Fe}, \mathrm{Sr}, \mathrm{Y}$ II, Zr II, Ba II, Ce) \\
\hline Mishenina et al. (2003) & 95 & $(\mathrm{Fe}, \mathrm{O}, \mathrm{Na})$ \\
\hline Mishenina et al. $(\overline{\overline{2004}})$ & 173 & $(\mathrm{Fe}, \mathrm{Mg}, \mathrm{Si}, \mathrm{Ni})$ \\
\hline Mishenina et al. $(\overline{2008})$ & 129 & $(\mathrm{Fe}, \mathrm{O}, \mathrm{Mg}, \mathrm{Si}, \mathrm{Ti})$ \\
\hline Mishenina et al. $(\overline{2011 b})$ & 142 & $(\mathrm{Fe}, \mathrm{Na}, \mathrm{Al}, \mathrm{Cu}, \mathrm{Zn})$ \\
\hline $\begin{array}{l}\text { Neuforge-Verheecke \& Magain } \\
\text { (1997) }\end{array}$ & 2 & $\begin{array}{l}\text { (C, N, O, Al, Si, Ca, Sc II, Ti, V, Cr, Cr II, Mn, Fe, Co, Ni, Y II, Zr II, Eu } \\
\text { II) }\end{array}$ \\
\hline Neves et al. (2009) & 443 & (Fe, Si, Ca, Sc, Sc II, Ti, Ti II, V, Cr, Cr II, Mn, Co, Ni, Na, Mg, Al) \\
\hline
\end{tabular}




\begin{tabular}{|c|c|c|}
\hline Nissen \& Schuster 1997$)$ & 19 & $(\mathrm{Fe}, \mathrm{O}, \mathrm{Mg}, \mathrm{Si}, \mathrm{Ca}, \mathrm{Ti}, \mathrm{Cr}, \mathrm{Ni}, \mathrm{Na}, \mathrm{Y}, \mathrm{Ba} \mathrm{II})$ \\
\hline Nissen \& Schuster 2010 & 43 & $(\mathrm{Fe}, \mathrm{Na}, \mathrm{Mg}, \mathrm{Si}, \mathrm{Ca}, \mathrm{Ti}, \mathrm{Cr}, \mathrm{Ni})$ \\
\hline Nissen \& Schuster 2011 & 36 & (Fe, Mn, Cu, Zn, Y II, Ba II) \\
\hline Petigura \& Marcy (2011) & 914 & $(\mathrm{Fe}, \mathrm{C}, \mathrm{O})$ \\
\hline Porto de Mello et al. (2008) & 2 & (Fe, Na, Mg, Si, Ca, Sc, Ti, V, Cr, Mn, Co, Ni, Cu, Y II, Ba II) \\
\hline Ramírez et al. (2007) & 523 & $(\mathrm{Fe}, \mathrm{O})$ \\
\hline Ramírez et al. $(\overline{2009})$ & 64 & $(\mathrm{Fe}, \mathrm{C}, \mathrm{O}, \mathrm{Na}, \mathrm{Al}, \mathrm{Si}, \mathrm{S}, \mathrm{Ca}, \mathrm{Sc}, \mathrm{Ti}, \mathrm{V}, \mathrm{Cr}, \mathrm{Mn}, \mathrm{Ni}, \mathrm{Cu}, \mathrm{Zn}, \mathrm{Y}$ II, Zr II, Ba II) \\
\hline Recio-Blanco et al. (2012) & 9 & $(\mathrm{Fe}, \mathrm{Y}, \mathrm{Zr})$ \\
\hline Reddy et al. $(2003)$ & 179 & $\begin{array}{l}\text { (Fe, C, N, O, Na, Mg, Al, Si, S, K, Ca, Sc II, Ti, V, Cr II, Mn, Co, Ni, Cu, } \\
\text { Zn, Sr, Y II, Ba II, Zr II, Ce, Nd, Eu) }\end{array}$ \\
\hline Reddy et al. (2006) & 171 & $\begin{array}{l}\text { (Fe, C, O, Na, Mg, Al, Si, Ca, Sc II, Ti, V, Cr II, Mn, Co, Ni, Cu, Zn, Y II, } \\
\text { Ba II, Ce, Nd, Eu) }\end{array}$ \\
\hline Sadakane et al. (2002) & 12 & $\begin{array}{l}\text { (C, N, O, Na, Mg, Al, Si, S, K, Ca, Sc, Sc II, Ti, T III, V, Cr, Cr II, Mn, Fe, } \\
\text { Co, Ni, Cu, Zn, Sr, Y II, Ba II, Ce II, Nd II, Eu II) }\end{array}$ \\
\hline Schuler et al. $(2011)$ & 10 & $(\mathrm{C}, \mathrm{N}, \mathrm{O}, \mathrm{Na}, \mathrm{Mg}, \mathrm{Al}, \mathrm{Si}, \mathrm{S}, \mathrm{Ca}, \mathrm{Sc}, \mathrm{Ti}, \mathrm{V}, \mathrm{Cr}, \mathrm{Mn}, \mathrm{Fe}, \mathrm{Co}, \mathrm{Ni}, \mathrm{Zn})$ \\
\hline Shi et al. $(2004)$ & 97 & $(\mathrm{Fe}, \mathrm{Na})$ \\
\hline \begin{tabular}{|l|l} 
Takeda \& Honda & $(2005)$ \\
and Takeda et al. & $(2007)$
\end{tabular} & 159 & $\begin{array}{l}\text { (Fe, C, N, O, Na, Mg, Al, Si, S, Ca, Sc, Sc II, Ti, Ti II, V, V II, Cr, Cr II, } \\
\mathrm{Mn}, \mathrm{Co}, \mathrm{Ni}, \mathrm{Cu}, \mathrm{Zn} \text { ) }\end{array}$ \\
\hline Thevenin (1998) & 663 & $\begin{array}{l}\text { (Li, O, Na, Mg, Al, Si, Ca, Sc, Ti, V, Cr, Mn, Fe, Co, Ni, Sr, Y, Zr, Mo, Ba } \\
\text { II, La, Ce, Nd, Sm, Eu) }\end{array}$ \\
\hline Trevisan et al. (2011) & 64 & $(\mathrm{Fe}, \mathrm{Ca}, \mathrm{Si}, \mathrm{Ti}, \mathrm{C}, \mathrm{Ni}, \mathrm{O}, \mathrm{Mg})$ \\
\hline Valenti \& Fischer (2005) & 1002 & $(\mathrm{Na}, \mathrm{Si}, \mathrm{Ti}, \mathrm{Fe}, \mathrm{Ni})$ \\
\hline Wang et al. $(2011)$ & 37 & ( $\mathrm{Fe}, \mathrm{Al}, \mathrm{Mg}, \mathrm{Si}, \mathrm{K}, \mathrm{Ca}, \mathrm{V}, \mathrm{Cr}, \mathrm{Sc}$ II, Y, Ba II, Eu II, La II, Ni, Ti) \\
\hline Zhang \& Zhao (2006) & 31 & (Fe, O, Mg, Si, Ca, Ti, Na, Al, Sc, V, Cr, Mn, Ni, Ba II) \\
\hline Zhao et al. (2002) & 12 & (Fe, C, O, Na, Mg, Al, Si, S, K, Ca, Sc II, Ti, V, Cr, Mn, Ni, Ba II) \\
\hline
\end{tabular}

Table 2:: Literature sources used in Hypatia, with the number of stars that matched the criteria and element abundances measured. 
$-51-$

\begin{tabular}{|c|c|c|c|c|c|c|c|c|c|c|c|c|c|c|}
\hline 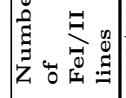 & 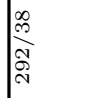 & $\frac{4}{z}$ & $\begin{array}{l}0 \\
\stackrel{0}{0} \\
0 \\
\end{array}$ & $\frac{1}{8}$ & $\frac{10}{\infty}$ & ळొ & $\frac{N}{\overparen{N}}$ & $\frac{\mathbb{N}}{\stackrel{N}{8}}$ & $\stackrel{\hat{}}{\stackrel{\sigma}{\sigma}}$ & 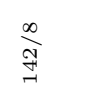 & 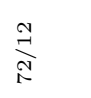 & 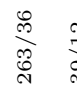 & 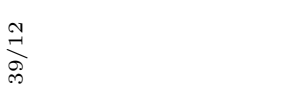 & $\underset{\widetilde{\infty}}{\stackrel{N}{\alpha}}$ \\
\hline 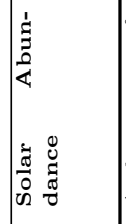 & 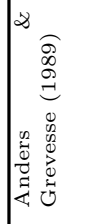 & 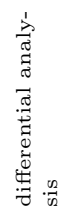 & 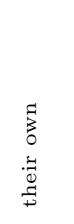 & 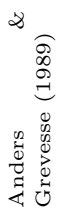 & 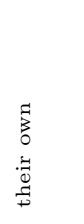 & 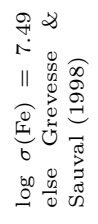 & 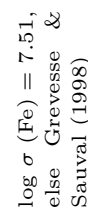 & & 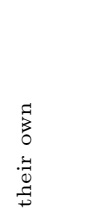 & 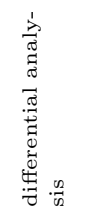 & 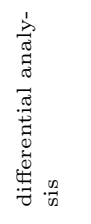 & 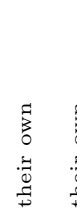 & 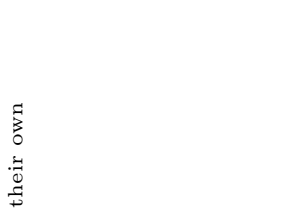 & 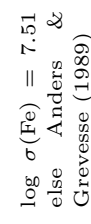 \\
\hline 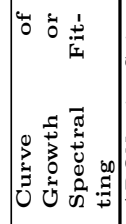 & 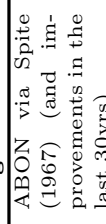 & 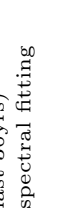 & 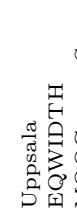 & 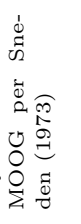 & $\begin{array}{l}0 \\
0 \\
0 \\
\\
\Sigma\end{array}$ & 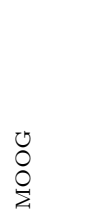 & $\begin{array}{l}5 \\
0 \\
0 \\
\vdots \\
\end{array}$ & $\begin{array}{l}5 \\
0 \\
0 \\
\vdots \\
\end{array}$ & 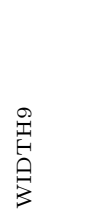 & 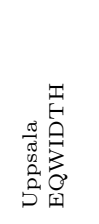 & $\begin{array}{l}0 \\
0 \\
0 \\
\vdots\end{array}$ & $\begin{array}{ll}0 & \zeta \\
0 & \vdots \\
\vdots & \vdots\end{array}$ & 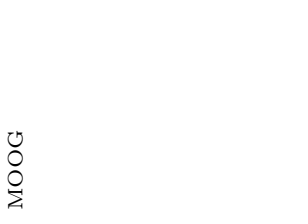 & 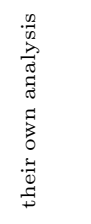 \\
\hline 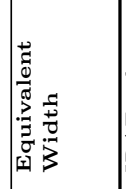 & 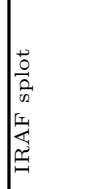 & 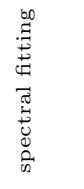 & 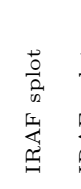 & 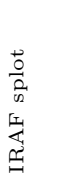 & 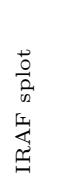 & 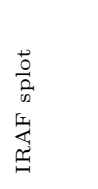 & 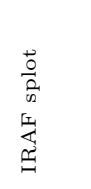 & 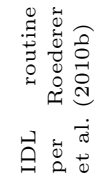 & 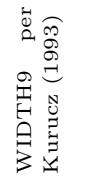 & 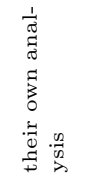 & 鹤 & 留 & 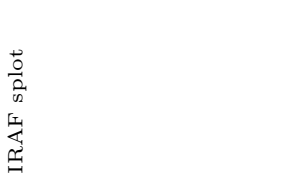 & 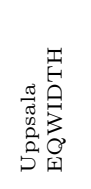 \\
\hline 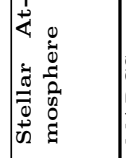 & 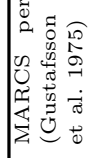 & 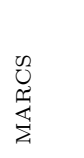 & 品 & 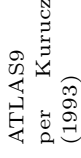 & & 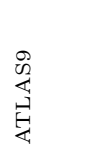 & 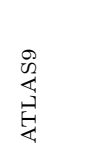 & 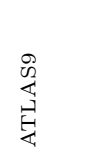 & 岁 & 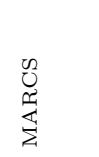 & 䚻 & 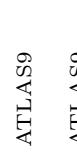 & 总 & 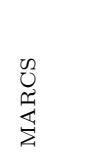 \\
\hline 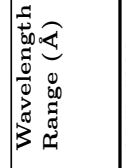 & $\mid$ & $\begin{array}{l}0 \\
0 \\
0 \\
0 \\
0 \\
0 \\
0 \\
0 \\
0 \\
0 \\
0 \\
0 \\
0\end{array}$ & $\begin{array}{l}0 \\
\stackrel{0}{0} \\
0 \\
0 \\
0 \\
0 \\
\infty\end{array}$ & $\begin{array}{l}0 \\
0 \\
0 \\
0 \\
0 \\
0 \\
\infty \\
\infty\end{array}$ & $\begin{array}{l}: \\
\vdots \\
\vdots \\
\vdots \\
\vdots \\
0\end{array}$ & 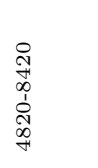 & 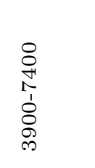 & 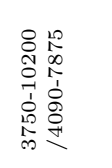 & $\begin{array}{l}0 \\
0 \\
0 \\
0 \\
0.0 \\
1.0 \\
10\end{array}$ & $\begin{array}{l}0 \\
0 \\
\infty \\
0 \\
0 \\
0 \\
1.0 \\
1.0\end{array}$ & $\begin{array}{l}19 \\
0 \\
0 \\
0 \\
10 \\
0 \\
0 \\
\infty\end{array}$ & 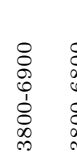 & $\begin{array}{l}8 \\
0 \\
0 \\
\vdots \\
0 \\
\infty \\
\infty\end{array}$ & 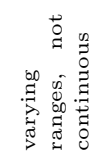 \\
\hline 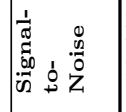 & 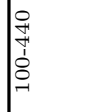 & $\begin{array}{l}8 \\
0 \\
\vdots \\
0 \\
\text { 吕 }\end{array}$ & $\begin{array}{l}\stackrel{8}{\infty} \\
\wedge\end{array}$ & 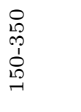 & 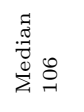 & 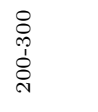 & $\begin{array}{l}0 \\
\stackrel{D}{0} \\
\text { 1. } \\
\stackrel{0}{0} \\
-1\end{array}$ & 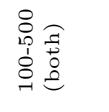 & $\begin{array}{l}8 \\
\text { o } \\
0 \\
0 \\
0 \\
0\end{array}$ & 品 & $\begin{array}{l}\stackrel{\text { N }}{\wedge} \\
\wedge\end{array}$ & $\stackrel{9}{g}$ & 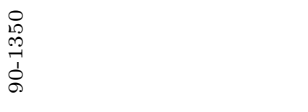 & 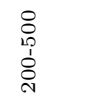 \\
\hline $\begin{array}{lll}1 & & \\
0 & & \\
0 \\
0 \\
0\end{array}$ & 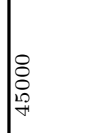 & 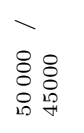 & 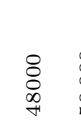 & $\begin{array}{l}8 \\
\vdots \\
\vdots \\
10\end{array}$ & 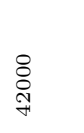 & $\begin{array}{l}\text { ¿े } \\
\vdots \\
\infty\end{array}$ & 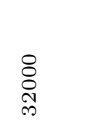 & 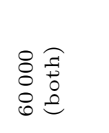 & 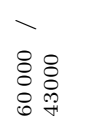 & \begin{tabular}{l}
8 \\
8 \\
8 \\
\hdashline
\end{tabular} & ষ⿳亠े冋ౖ & 总 & ^ & $\begin{array}{l}- \\
8 \\
\circ\end{array}$ \\
\hline 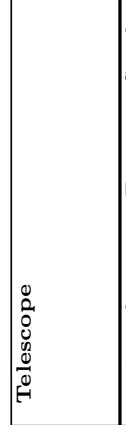 & 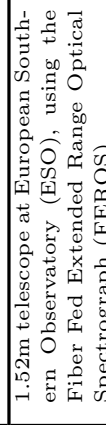 & & 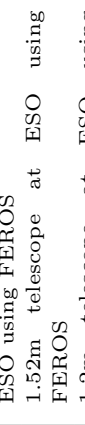 & 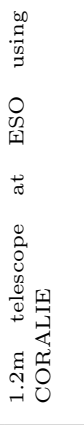 & 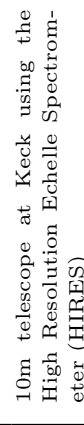 & 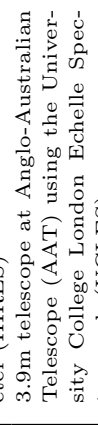 & 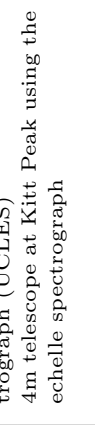 & 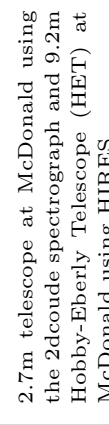 & 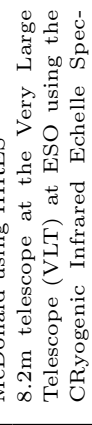 & 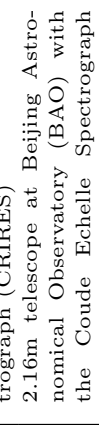 & 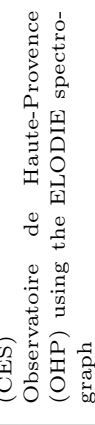 & 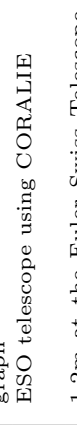 & 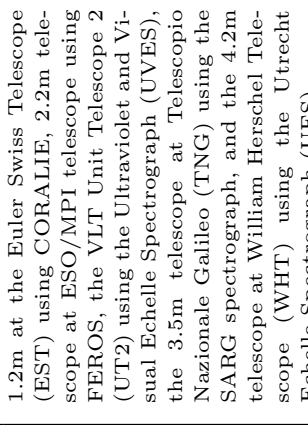 & 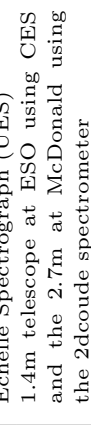 \\
\hline$\frac{\infty 00}{\sigma}$ & 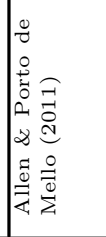 & 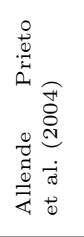 & 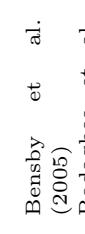 & 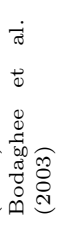 & 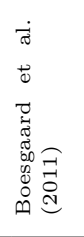 & 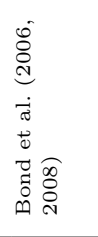 & 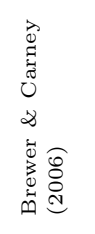 & 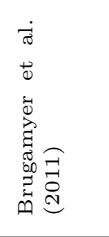 & 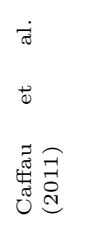 & 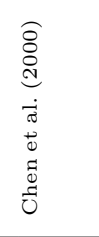 & 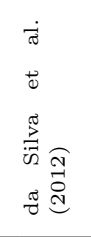 & 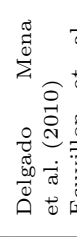 & 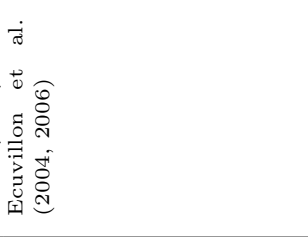 & 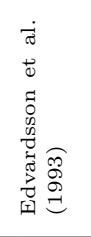 \\
\hline
\end{tabular}


$-52-$

\begin{tabular}{|c|c|c|c|c|c|c|c|c|c|c|}
\hline 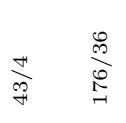 & $\begin{array}{l}\circ \\
\stackrel{2}{\circ}\end{array}$ & 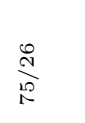 & $\begin{array}{l}0 \\
\text { i }\end{array}$ & 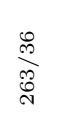 & $\begin{array}{l}\vec{\exists} \\
\stackrel{\overrightarrow{0}}{0}\end{array}$ & 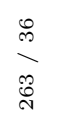 & $\begin{array}{l}\stackrel{1}{\rightleftharpoons} \\
\stackrel{f}{Z}\end{array}$ & $\stackrel{0}{=} \underset{-\infty}{\infty}$ & $\frac{7}{3}$ & $\underset{\infty}{\stackrel{N}{N}}$ \\
\hline 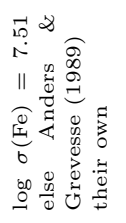 & 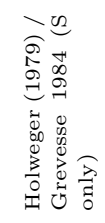 & 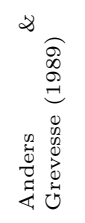 & 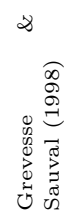 & 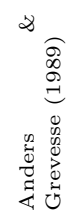 & 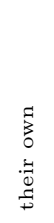 & 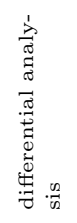 & 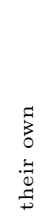 & 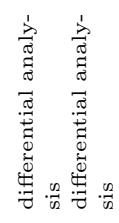 & & \\
\hline
\end{tabular}

\begin{tabular}{|c|c|c|c|c|c|c|c|c|c|c|c|c|}
\hline 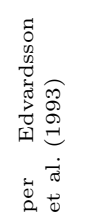 & 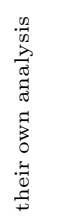 & $\begin{array}{l}\text { ñ } \\
\text { o } \\
\text { qu }\end{array}$ & \begin{tabular}{l}
0 \\
0 \\
0 \\
\multirow{2}{*}{}
\end{tabular} & 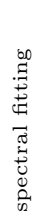 & $\begin{array}{l}0 \\
0 \\
\stackrel{0}{\Sigma}\end{array}$ & $\begin{array}{l}0 \\
\stackrel{0}{0} \\
\frac{0}{2}\end{array}$ & $\begin{array}{l}0 \\
0 \\
0 \\
\Sigma\end{array}$ & 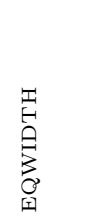 & 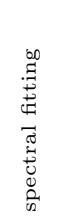 & 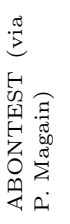 & 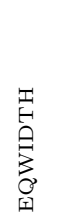 & $\begin{array}{l}0 \\
\stackrel{0}{0} \\
\stackrel{0}{2}\end{array}$ \\
\hline 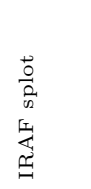 & 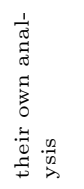 & $\frac{\varangle}{z}$ & 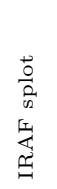 & 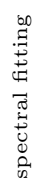 & 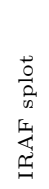 & 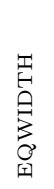 & 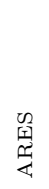 & 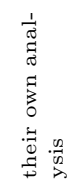 & 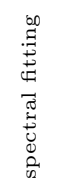 & 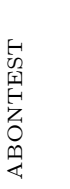 & 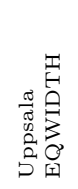 & 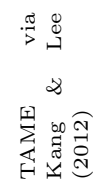 \\
\hline
\end{tabular}

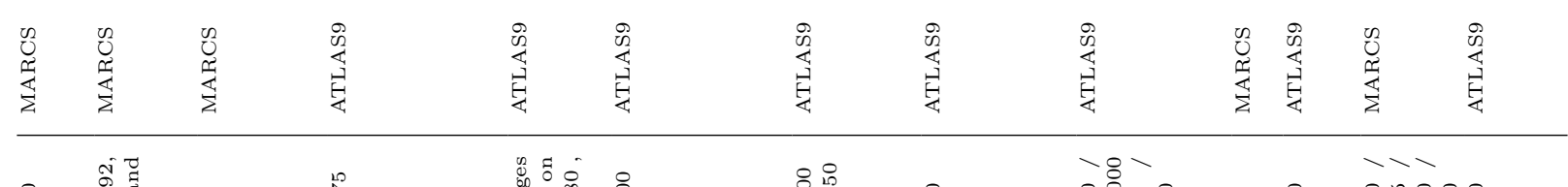

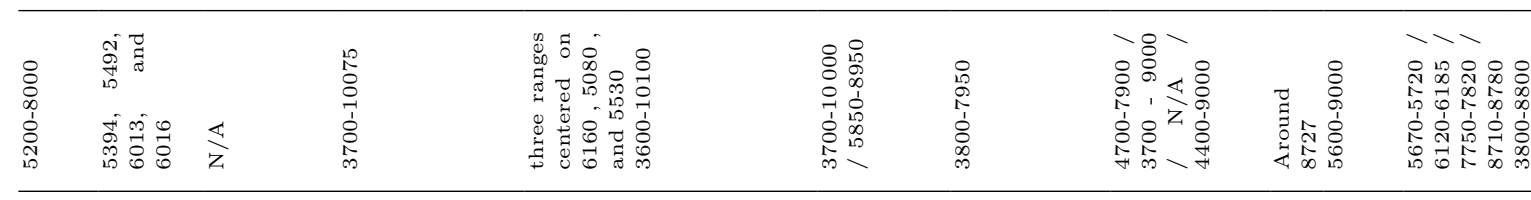

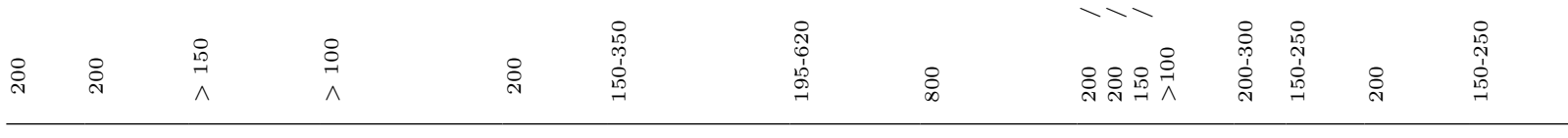

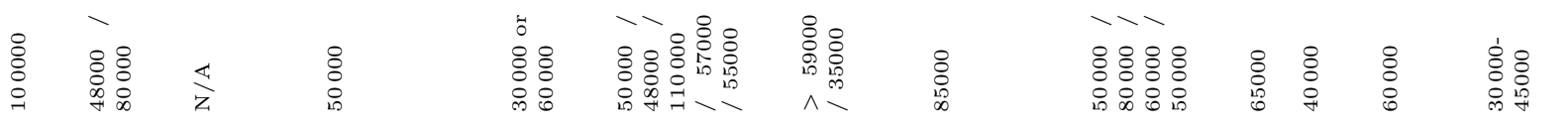

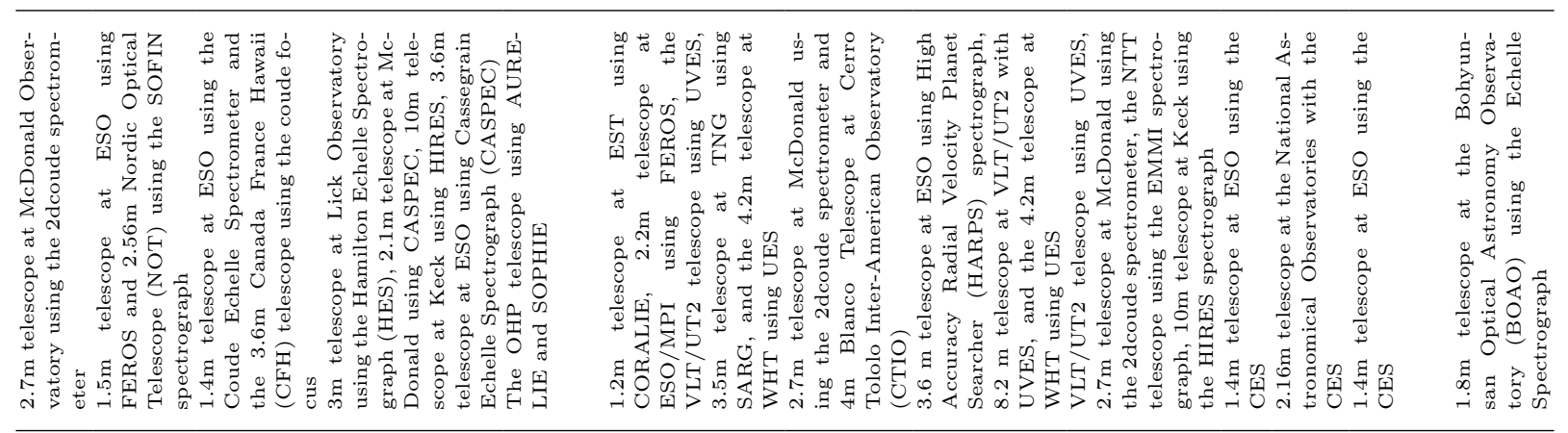

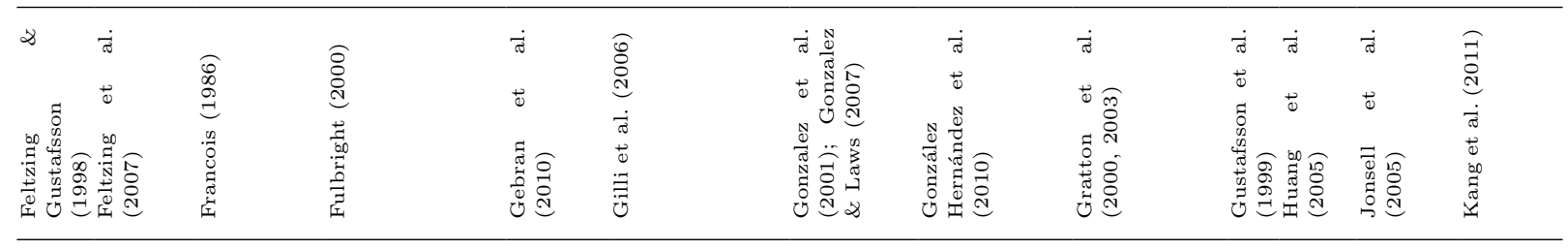


$-53-$

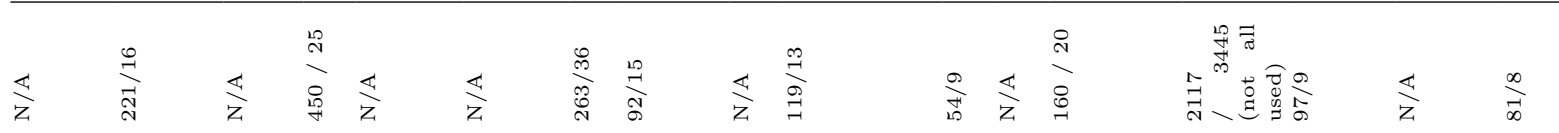

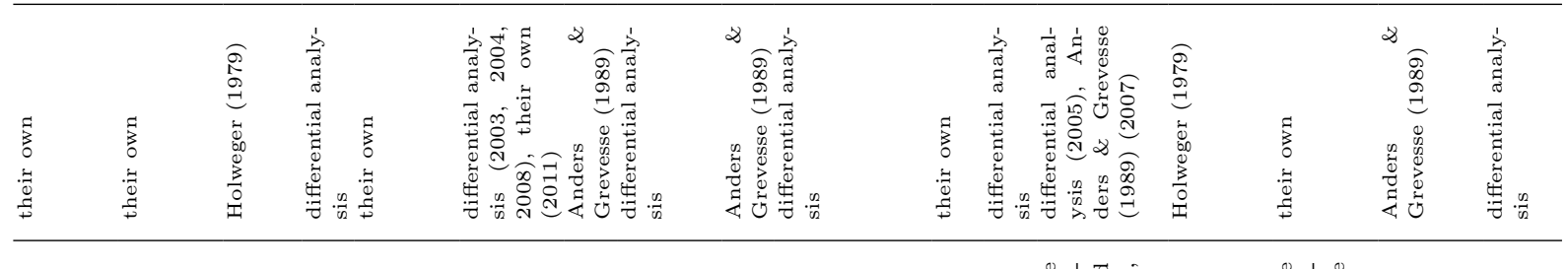

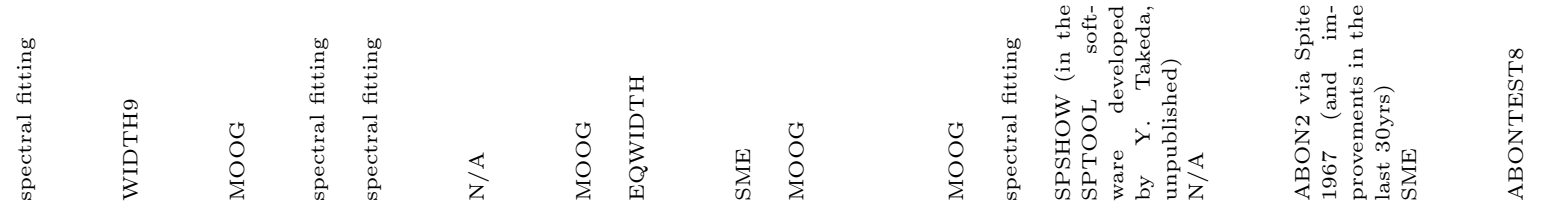

\begin{tabular}{|c|c|c|c|c|c|c|c|c|c|c|c|c|c|c|c|c|}
\hline 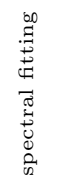 & 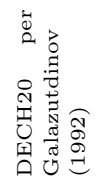 & 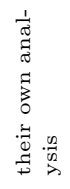 & 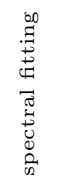 & 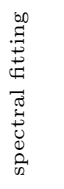 & 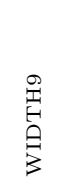 & 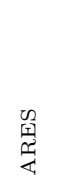 & 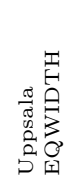 & 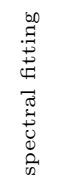 & 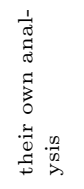 & 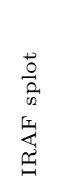 & 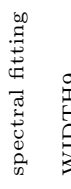 & 蛋 & 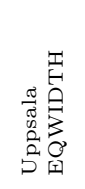 & $\begin{array}{l}\text { 罂 } \\
\text { 出 }\end{array}$ & 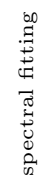 & $\begin{array}{l}\infty \\
6 \\
0 \\
9 \\
\vdots \\
0 \\
0 \\
0\end{array}$ \\
\hline
\end{tabular}

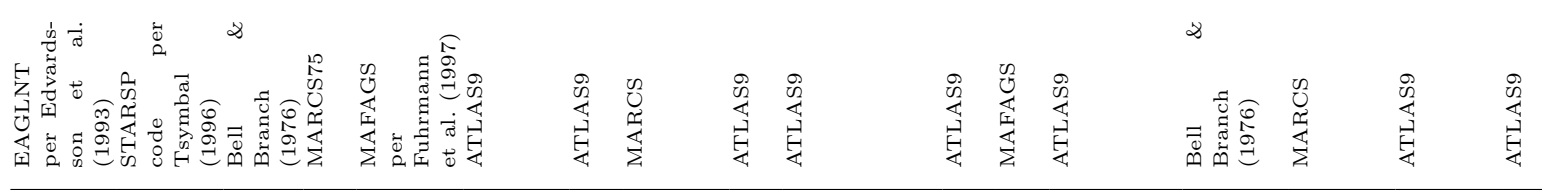

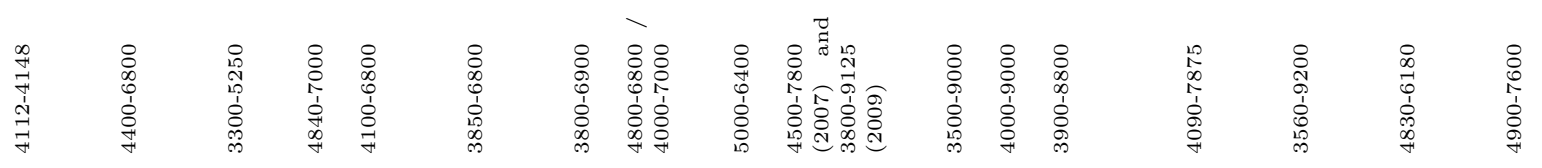

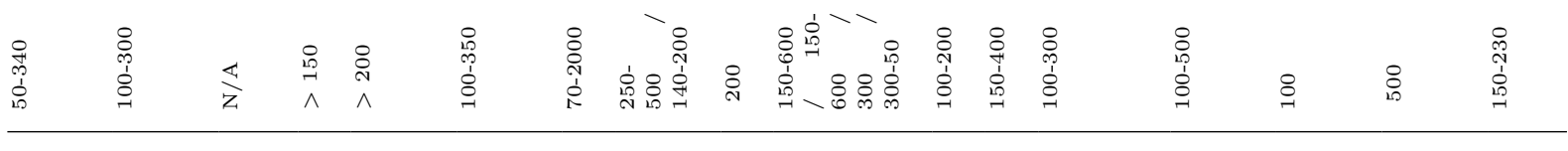

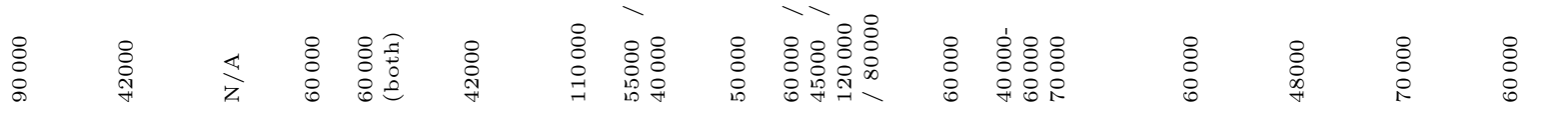

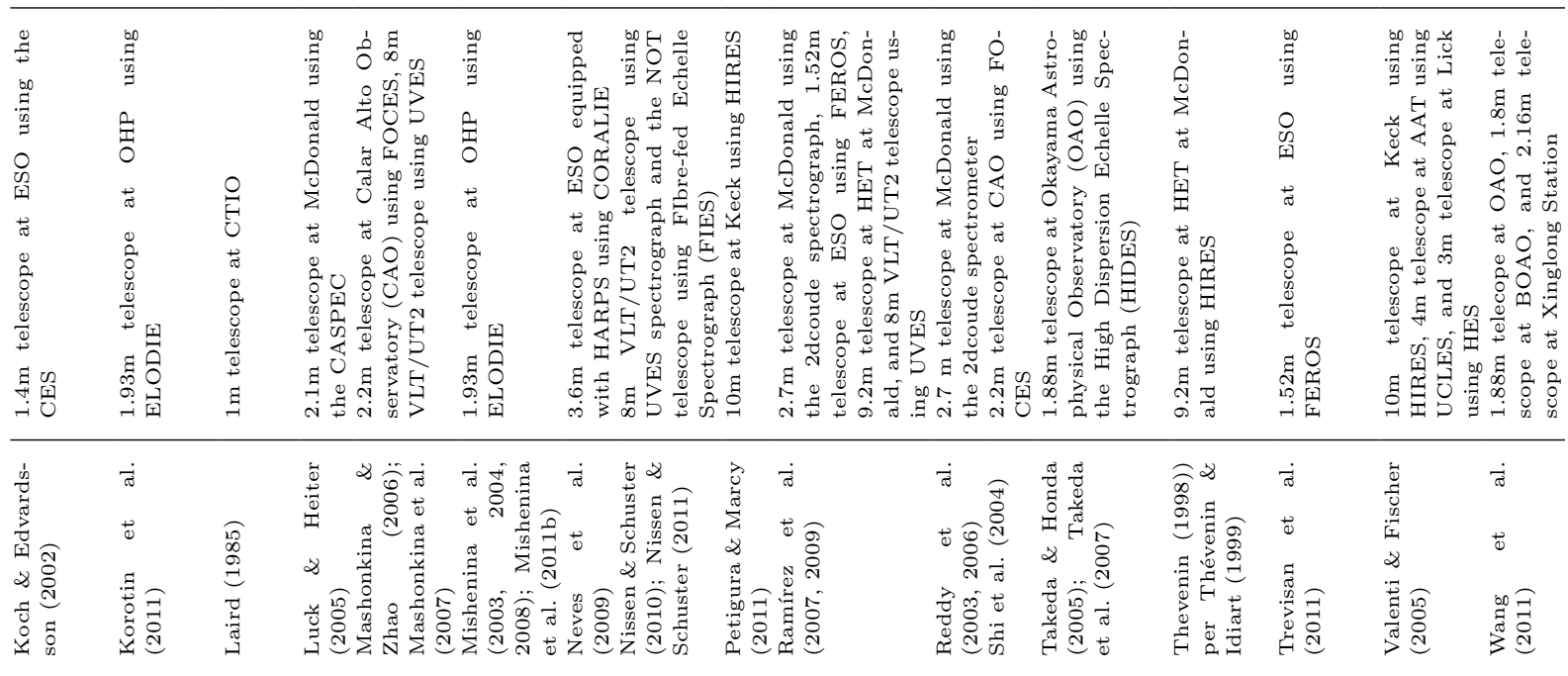


7

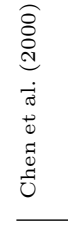

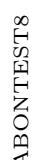

$\frac{9}{4}$

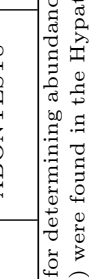

甚

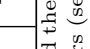

○

赵

客噌

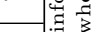

줄

品

$\longrightarrow$

$\therefore$

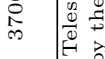

Ð

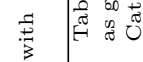

安

范

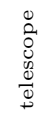

量

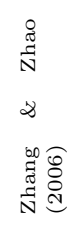




\begin{tabular}{|c|c|c|c|c|c|c|c|c|c|}
\hline \multirow[t]{2}{*}{ Element } & \multicolumn{3}{|c|}{$[\mathrm{Fe} / \mathrm{H}]=-0.4 \mathrm{dex}$} & \multicolumn{3}{|c|}{$[\mathrm{Fe} / \mathrm{H}]=0.0 \mathrm{dex}$} & \multicolumn{3}{|c|}{$[\mathrm{Fe} / \mathrm{H}]=0.4 \mathrm{dex}$} \\
\hline & $16 \%$ & Med & $84 \%$ & $16 \%$ & Med & $84 \%$ & $16 \%$ & Med & $84 \%$ \\
\hline $\bar{C}$ & 0.16 & 0.25 & 0.34 & -0.18 & -0.07 & 0.09 & -0.24 & -0.15 & -0.07 \\
\hline $\mathrm{O}$ & 0.12 & 0.28 & 0.40 & -0.34 & -0.09 & 0.06 & -0.48 & -0.36 & -0.25 \\
\hline $\mathrm{Na}$ & -0.06 & 0.01 & 0.07 & -0.23 & -0.16 & 0.03 & -0.26 & -0.16 & -0.07 \\
\hline $\mathrm{Mg}$ & 0.01 & 0.07 & 0.15 & -0.17 & -0.07 & 0.08 & -0.19 & -0.11 & -0.02 \\
\hline $\mathrm{Al}$ & -0.15 & -0.09 & 0.03 & -0.17 & -0.08 & 0.02 & -0.17 & -0.12 & -0.07 \\
\hline $\mathrm{Si}$ & 0.01 & 0.12 & 0.15 & -0.18 & -0.09 & 0.05 & -0.25 & -0.20 & -0.09 \\
\hline K & $\mathrm{N} / \mathrm{A}$ & 0.34 & $\mathrm{~N} / \mathrm{A}$ & $\mathrm{N} / \mathrm{A}$ & -0.00 & $\mathrm{~N} / \mathrm{A}$ & $\mathrm{N} / \mathrm{A}$ & $\mathrm{N} / \mathrm{A}$ & $\mathrm{N} / \mathrm{A}$ \\
\hline $\mathrm{Ca}$ & 0.00 & 0.05 & 0.09 & -0.13 & -0.02 & 0.04 & -0.21 & -0.12 & -0.02 \\
\hline $\mathrm{Sc}$ & -0.04 & 0.03 & 0.27 & -0.14 & -0.05 & 0.09 & -0.12 & -0.10 & 0.01 \\
\hline ScII & 0.09 & 0.18 & 0.23 & -0.22 & -0.16 & 0.06 & -0.16 & -0.11 & -0.07 \\
\hline $\mathrm{Ti}$ & -0.05 & 0.01 & 0.09 & -0.12 & -0.04 & 0.04 & -0.21 & -0.16 & -0.06 \\
\hline TiII & $\mathrm{N} / \mathrm{A}$ & 0.01 & $\mathrm{~N} / \mathrm{A}$ & $\mathrm{N} / \mathrm{A}$ & -0.15 & $\mathrm{~N} / \mathrm{A}$ & $\mathrm{N} / \mathrm{A}$ & -0.17 & $\mathrm{~N} / \mathrm{A}$ \\
\hline V & -0.14 & -0.07 & 0.06 & -0.11 & -0.05 & 0.10 & -0.06 & 0.00 & 0.18 \\
\hline $\mathrm{Cr}$ & -0.14 & -0.06 & 0.03 & -0.17 & -0.09 & -0.01 & -0.18 & -0.17 & -0.14 \\
\hline CrII & -0.38 & -0.33 & -0.29 & -0.53 & -0.51 & -0.45 & -0.56 & -0.54 & -0.52 \\
\hline $\mathrm{Mn}$ & -0.39 & -0.31 & -0.22 & -0.37 & -0.25 & -0.10 & -0.23 & -0.20 & -0.15 \\
\hline Co & -0.06 & -0.02 & 0.06 & -0.18 & -0.08 & 0.01 & -0.06 & -0.02 & 0.11 \\
\hline $\mathrm{Ni}$ & -0.06 & -0.04 & -0.00 & -0.22 & -0.15 & -0.01 & -0.22 & -0.16 & -0.09 \\
\hline $\mathrm{Cu}$ & $\mathrm{N} / \mathrm{A}$ & -0.21 & $\mathrm{~N} / \mathrm{A}$ & $\mathrm{N} / \mathrm{A}$ & -0.12 & $\mathrm{~N} / \mathrm{A}$ & $\mathrm{N} / \mathrm{A}$ & $\mathrm{N} / \mathrm{A}$ & $\mathrm{N} / \mathrm{A}$ \\
\hline $\mathrm{Zn}$ & $\mathrm{N} / \mathrm{A}$ & -0.15 & $\mathrm{~N} / \mathrm{A}$ & $\mathrm{N} / \mathrm{A}$ & -0.13 & $\mathrm{~N} / \mathrm{A}$ & $\mathrm{N} / \mathrm{A}$ & $\mathrm{N} / \mathrm{A}$ & $\mathrm{N} / \mathrm{A}$ \\
\hline $\mathrm{Sr}$ & $\mathrm{N} / \mathrm{A}$ & -0.27 & $\mathrm{~N} / \mathrm{A}$ & $\mathrm{N} / \mathrm{A}$ & -0.18 & $\mathrm{~N} / \mathrm{A}$ & $\mathrm{N} / \mathrm{A}$ & $\mathrm{N} / \mathrm{A}$ & $\mathrm{N} / \mathrm{A}$ \\
\hline $\mathrm{Y}$ & $\mathrm{N} / \mathrm{A}$ & -0.05 & $\mathrm{~N} / \mathrm{A}$ & $\mathrm{N} / \mathrm{A}$ & 0.02 & $\mathrm{~N} / \mathrm{A}$ & $\mathrm{N} / \mathrm{A}$ & $\mathrm{N} / \mathrm{A}$ & $\mathrm{N} / \mathrm{A}$ \\
\hline YII & $\mathrm{N} / \mathrm{A}$ & -0.12 & $\mathrm{~N} / \mathrm{A}$ & $\mathrm{N} / \mathrm{A}$ & -0.03 & $\mathrm{~N} / \mathrm{A}$ & $\mathrm{N} / \mathrm{A}$ & $\mathrm{N} / \mathrm{A}$ & $\mathrm{N} / \mathrm{A}$ \\
\hline $\mathrm{Zr}$ & $\mathrm{N} / \mathrm{A}$ & 0.08 & $\mathrm{~N} / \mathrm{A}$ & $\mathrm{N} / \mathrm{A}$ & -0.06 & $\mathrm{~N} / \mathrm{A}$ & $\mathrm{N} / \mathrm{A}$ & $\mathrm{N} / \mathrm{A}$ & $\mathrm{N} / \mathrm{A}$ \\
\hline ZrII & $\mathrm{N} / \mathrm{A}$ & -0.12 & $\mathrm{~N} / \mathrm{A}$ & $\mathrm{N} / \mathrm{A}$ & 0.01 & $\mathrm{~N} / \mathrm{A}$ & $\mathrm{N} / \mathrm{A}$ & $\mathrm{N} / \mathrm{A}$ & $\mathrm{N} / \mathrm{A}$ \\
\hline BaII & -0.09 & -0.03 & 0.16 & -0.13 & -0.02 & 0.14 & $\mathrm{~N} / \mathrm{A}$ & $\mathrm{N} / \mathrm{A}$ & $\mathrm{N} / \mathrm{A}$ \\
\hline $\mathrm{Ce}$ & $\mathrm{N} / \mathrm{A}$ & -0.01 & $\mathrm{~N} / \mathrm{A}$ & $\mathrm{N} / \mathrm{A}$ & 0.05 & $\mathrm{~N} / \mathrm{A}$ & $\mathrm{N} / \mathrm{A}$ & $\mathrm{N} / \mathrm{A}$ & $\mathrm{N} / \mathrm{A}$ \\
\hline $\mathrm{Nd}$ & $\mathrm{N} / \mathrm{A}$ & 0.05 & $\mathrm{~N} / \mathrm{A}$ & $\mathrm{N} / \mathrm{A}$ & 0.05 & $\mathrm{~N} / \mathrm{A}$ & $\mathrm{N} / \mathrm{A}$ & $\mathrm{N} / \mathrm{A}$ & $\mathrm{N} / \mathrm{A}$ \\
\hline $\mathrm{Eu}$ & $\mathrm{N} / \mathrm{A}$ & 0.12 & $\mathrm{~N} / \mathrm{A}$ & $\mathrm{N} / \mathrm{A}$ & -0.04 & $\mathrm{~N} / \mathrm{A}$ & $\mathrm{N} / \mathrm{A}$ & $\mathrm{N} / \mathrm{A}$ & $\mathrm{N} / \mathrm{A}$ \\
\hline
\end{tabular}

Table 4:: Median and percentile $1 \sigma$ values for all elements with trend lines.

Table 5: Number of Thin-Disk Stars in Hypatia with Spread Less than error bars

\begin{tabular}{c|c|c|c|c|c|c|c|c|c|c|c|c|c|c|c|c}
\hline \hline $\mathbf{L i}$ & $\mathbf{B e}$ & $\mathbf{C}$ & $\mathbf{N}$ & $\mathbf{O}$ & $\mathbf{N a}$ & $\mathbf{M g}$ & $\mathbf{A l}$ & $\mathbf{S i}$ & $\mathbf{P}$ & $\mathbf{S}$ & $\mathbf{K}$ & $\mathbf{C a}$ & $\mathbf{C a I I}$ & $\mathbf{S c}$ & $\mathbf{S c I I}$ & $\mathbf{T i}$ \\
54 & 5 & 808 & 54 & 933 & 907 & 578 & 523 & 1098 & 1 & 162 & 139 & 657 & 8 & 381 & 386 & 1064 \\
\hline $\mathbf{T i I I}$ & $\mathbf{V}$ & $\mathbf{V I I}$ & $\mathbf{C r}$ & $\mathbf{C r I I}$ & $\mathbf{M n}$ & $\mathbf{F e}$ & $\mathbf{C o}$ & $\mathbf{N i}$ & $\mathbf{C u}$ & $\mathbf{Z n}$ & $\mathbf{S r}$ & $\mathbf{S r I I}$ & $\mathbf{Y}$ & $\mathbf{Y I I}$ & $\mathbf{Z r}$ & $\mathbf{Z r I I}$ \\
227 & 564 & 13 & 483 & 329 & 512 & 1713 & 454 & 1035 & 242 & 203 & 119 & 25 & 191 & 210 & 96 & 188 \\
\hline $\mathbf{M o}$ & $\mathbf{R u}$ & $\mathbf{B a I I}$ & $\mathbf{L a}$ & $\mathbf{L a I I}$ & $\mathbf{C e}$ & $\mathbf{C e I I}$ & $\mathbf{P r}$ & $\mathbf{N d}$ & $\mathbf{S m}$ & $\mathbf{E u}$ & $\mathbf{E u I I}$ & $\mathbf{G d}$ & $\mathbf{D y}$ & $\mathbf{H f}$ & $\mathbf{P b}$ & \\
48 & 19 & 301 & 73 & 39 & 237 & 5 & 75 & 203 & 29 & 119 & 34 & 62 & 17 & 73 & 17 & \\
\hline \hline
\end{tabular}

\section{REFERENCES}

Adibekyan, V. Z., et al. 2013, A\&A, 554, A44

Allen, D. M., \& Porto de Mello, G. F. 2011, A\&A, 525, 63 
Table 6: Scale Heights and 90\% Heights for Ensembles Above and Below [X/Fe] Gap

\begin{tabular}{lcccc}
\hline Element & $h_{a} \mathbf{p c}$ & $h_{b} \mathbf{p c}$ & $h_{90 a} \mathbf{p c}$ & $h_{90 b} \mathbf{p c}$ \\
\hline $\mathrm{Mg}$ & 35 & 22 & 76 & 39 \\
$\mathrm{Si}$ & 37 & 26 & 77 & 47 \\
$\mathrm{~S}$ & 39 & 34 & 68 & 57 \\
$\mathrm{Ca}$ & 38 & 24 & 78 & 40 \\
$\mathrm{Sc}$ & 39 & 21 & 76 & 36 \\
$\mathrm{Ti}$ & 32 & 29 & 71 & 50 \\
$\mathrm{Cr}$ & 37 & 22 & 68 & 37 \\
$\mathrm{Ni}$ & 40 & 28 & 80 & 50 \\
\hline
\end{tabular}

${ }^{a}$ Standard composition values relative to Lodders et al. (2009) solar

Table 7: Scale Heights and 90\% Heights for Ensembles Above and Below [X/Fe] Gap Without Neves et al. (2009)

\begin{tabular}{lcccc}
\hline Element & $h_{a} \mathbf{p c}$ & $h_{b} \mathbf{p c}$ & $h_{90 a} \mathbf{p c}$ & $h_{90 b} \mathbf{p c}$ \\
\hline $\mathrm{Mg}$ & 35 & 24 & 76 & 42 \\
$\mathrm{Si}$ & 36 & 27 & 77 & 48 \\
$\mathrm{~S}$ & 39 & 33 & 68 & 57 \\
$\mathrm{Ca}$ & 41 & 33 & 80 & 80 \\
$\mathrm{Sc}$ & 34 & 20 & 82 & 77 \\
$\mathrm{Ti}$ & 36 & 29 & 75 & 51 \\
$\mathrm{Cr}$ & 37 & 29 & 68 & 81 \\
$\mathrm{Ni}$ & 40 & 29 & 79 & 50 \\
\hline
\end{tabular}

${ }^{a}$ Standard composition values relative to Lodders et al. (2009) solar

Allende Prieto, C., Barklem, P. S., Lambert, D. L., \& Cunha, K. 2004, A\&A, 420, 183

Anders, E., \& Grevesse, N. 1989, Geochimica et Cosmochimica Acta, 53, 197

Anderson, E., \& Francis, C. 2012, Astronomy Letters, 38, 331

Arlandini, C., Käppeler, F., Wisshak, K., Gallino, R., Lugaro, M., Busso, M., \& Straniero, O. 1999, ApJ, 525, 886

Arnett, D. 1996, Supernovae and Nucleosynthesis: An Investigation of the History of Matter from the Big Bang to the Present (New Jersey: Princeton University Press)

Arnett, W. D., \& Thielemann, F.-K. 1985, ApJ, 295, 589

Arnould, M., Goriely, S., \& Takahashi, K. 2007, Physics Reports, 450, 97

Asplund, M., Grevesse, N., Sauval, A. J., \& Scott, P. 2009, ARA\&A, 47, 481

Beirão, P., Santos, N. C., Israelian, G., \& Mayor, M. 2005, A\&A, 438, 251

Bell, R. A., \& Branch, D. 1976, MNRAS, 175, 25 
Bennett, M. E., et al. 2012, ArXiv e-prints

Bensby, T., Feltzing, S., \& Lundström, I. 2003, A\&A, 410, 527

-. 2004, A\&A, 415, 155

Bensby, T., Feltzing, S., Lundström, I., \& Ilyin, I. 2005, A\&A, 433, 185

Bergemann, M. 2011, MNRAS, 413, 2184

Bergemann, M., \& Cescutti, G. 2010, A\&A, 522, A9

Bergemann, M., \& Gehren, T. 2007, A\&A, 473, 291

-. 2008, A\&A, 492, 823

Bergemann, M., Pickering, J. C., \& Gehren, T. 2010, MNRAS, 401, 1334

Bethe, H. A., \& Critchfield, C. L. 1938, PRB, 54, 862

Bidelman, W. P. 1960, PASP, 72, 24

Bodaghee, A., Santos, N. C., Israelian, G., \& Mayor, M. 2003, A\&A, 404, 715

Bodaghee, A., Santos, N. C., Israelian, G., \& Mayor, M. 2003, A\&A, 404, 715

Boesgaard, A. M., Rich, J. A., Levesque, E. M., \& Bowler, B. P. 2011, ApJ, 743, 140

Bond, J. C., Lauretta, D. S., Tinney, C. G., Butler, R. P., Marcy, G. W., Jones, H. R. A., Carter, B. D., \& Bailey, S. J. O. . J. 2008, ApJ, 682, 1234

Bond, J. C., Tinney, C. G., Butler, R. P., Jones, H. R. A., Marcy, G. W., Penny, A. J., \& Carter, B. D. 2006, MNRAS, 370, 163

Brewer, M.-M., \& Carney, B. W. 2006, AJ, 131, 431

Brugamyer, E., Dodson-Robinson, S. E., Cochran, W. D., \& Sneden, C. 2011, ApJ, submitted

Burbidge, E. M., Burbidge, G. R., Fowler, W. A., \& Hoyle, F. 1957, Reviews of Modern Physics, 29,547

Busso, M., Gallino, R., \& Wasserburg, G. J. 1999, ARA\&A, 37, 239

Caffau, E., Bonifacio, P., Faraggiana, R., \& Steffen, M. 2011, A\&A, 1107, 2657, submitted

Cameron, A. G. W. 1957, Stellar Evolution, Nuclear Astrophysics, and Nucleogenesis, Tech. rep., Chalk River Laboratory Report 41

Cameron, A. G. W. 1982, Ap\&SS, 82, 123 
Carbon, D. F., Barbuy, B., Kraft, R. P., Friel, E. D., \& Suntzeff, N. B. 1987, PASP, 99, 335

Carlson, R. W., Boyet, M., \& Horan, M. 2007, Science, 316, 1175

Carretta, E., Gratton, R. G., \& Sneden, C. 2000, A\&A, 356, 238

Castro, S., de Mello, G. P., \& da Silva, L. 1999, MNRAS, 305, 693

Castro, S., Rich, R. M., Grenon, M., Barbuy, B., \& McCarthy, J. K. 1997, AJ, 114, 376

Chen, Y. Q., Nissen, P. E., \& Zhao, G. 2004, A\&A, 425, 697

Chen, Y. Q., Nissen, P. E., Zhao, G., Zhang, H. W., \& Benoni, T. 2000, A\&AS, 141, 491

Chiappini, C. 2011, in EAS Publications Series, Vol. 45, EAS Publications Series, 293-298

Chiappini, C., Matteucci, F., \& Gratton, R. 1997, ApJ, 477, 765

Chieffi, A., \& Limongi, M. 2004, ApJ, 608, 405

Clayton, D. 2003, Handbook of Isotopes in the Cosmos (Cambridge University Press)

Clayton, D. D. 1968, Principles of stellar evolution and nucleosynthesis (New York: McGraw-Hill)

Couch, R. G., Schmiedekamp, A. B., \& Arnett, W. D. 1974, ApJ, 190, 95

da Silva, R., Porto de Mello, G. F., Milone, A. C., da Silva, L., Ribeiro, L. S., \& Rocha-Pinto, H. J. 2012, A\&A, 542, A84

Dauphas, N., et al. 2010, ApJ, 720, 1577

del Peloso, E. F., Cunha, K., da Silva, L., \& Porto de Mello, G. F. 2005, A\&A, 441, 1149

Delgado Mena, E., Israelian, G., González Hernández, J. I., Bond, J. C., Santos, N. C., Udry, S., \& Mayor, M. 2010, ApJ, 725, 2349

D’Orazi, V., Biazzo, K., Desidera, S., Covino, E., Andrievsky, S. M., \& Gratton, R. G. 2012, MNRAS, 423, 2789

Ecuvillon, A., Israelian, G., Santos, N. C., Mayor, M., Villar, V., \& Bihain, G. 2004, A\&A, 426, 619

Ecuvillon, A., Israelian, G., Santos, N. C., Shchukina, N. G., Mayor, M., \& Rebolo, R. 2006, A\&A, 445,633

Edvardsson, B., Andersen, J., Gustafsson, B., Lambert, D. L., Nissen, P. E., \& Tomkin, J. 1993, A\&A, 275, 101

Eggen, O. J., Lynden-Bell, D., \& Sandage, A. R. 1962, ApJ, 136, 748 
Ekström, S., Meynet, G., Chiappini, C., Hirschi, R., \& Maeder, A. 2008, A\&A, 489, 685

Ekström, S., et al. 2011, ArXiv e-prints

Feltzing, S., Fohlman, M., \& Bensby, T. 2007, A\&A, 467, 665

Feltzing, S., \& Gustafsson, B. 1998, A\&AS, 129, 237

Feltzing, S., Holmberg, J., \& Hurley, J. R. 2001, A\&A, 377, 911

Fenner, Y., Gibson, B. K., c Lee, H., Karakas, A. I., Lattanzio, J. C., Chieffi, A., Limongi, M., \& Yong, D. 2003, PASA, 20, 340

Francis, C., \& Anderson, E. 2009, New A, 14, 615

Francois, P. 1986, A\&A, 160, 264

-. 1987, A\&A, 176, 294

-. 1988, A\&A, 195, 226

Fuhrmann, K., Pfeiffer, M. J., \& Bernkopf, J. 1997, A\&A, 326, 1081

Fulbright, J. P. 2000, AJ, 120, 1841

Galazutdinov, G. 1992, in Preprints for the Special Astrophysical Observatory of the Russian Academy of Sciences, Vol. 92, 96

Galeev, A. I., Bikmaev, I. F., Musaev, F. A., \& Galazutdinov, G. A. 2004, Astronomy Reports, 48, 492

Gebran, M., Vick, M., Monier, R., \& Fossati, L. 2010, A\&A, 523, 71

Gibson, B. K., Fenner, Y., Renda, A., Kawata, D., \& Lee, H. 2003, PASA, 20, 401

Gilli, G., Israelian, G., Ecuvillon, A., Santos, N. C., \& Mayor, M. 2006, A\&A, 449, 723

Gonzalez, G., \& Laws, C. 2007, MNRAS, 378, 1141

Gonzalez, G., Laws, C., Tyagi, S., \& Reddy, B. E. 2001, AJ, 121, 432

González Hernández, J. I., Israelian, G., Santos, N. C., Sousa, S., Delgado-Mena, E., Neves, V., \& Udry, S. 2010, ApJ, 720, 1592

Goswami, A., \& Prantzos, N. 2000, A\&A, 359, 191

Gratton, R., Carretta, E., Matteucci, F., \& Sneden, C. 1996, in Astronomical Society of the Pacific Conference Series, Vol. 92, Formation of the Galactic Halo...Inside and Out, ed. H. L. Morrison \& A. Sarajedini, 307 
Gratton, R. G. 1989, A\&A, 208, 171

Gratton, R. G., Carretta, E., Claudi, R., Lucatello, S., \& Barbieri, M. 2003, A\&A, 404, 187

Gratton, R. G., \& Ortolani, S. 1986, A\&A, 169, 201

Gratton, R. G., \& Sneden, C. 1987, A\&A, 178, 179

Gratton, R. G., Sneden, C., Carretta, E., \& Bragaglia, A. 2000, A\&A, 354, 169

Grevesse, N., \& Sauval, A. J. 1998, Space Sci Rev, 85, 161

Gustafsson, B., Bell, R. A., Eriksson, K., \& Nordlund, A. 1975, A\&A, 42, 407

Gustafsson, B., Karlsson, T., Olsson, E., Edvardsson, B., \& Ryde, N. 1999, A\&A, 342, 426

Hauck, B., \& Berthet, S. 1991, in Precision Photometry: Astrophysics of the Galaxy eds. A.G.D. Philip, A.R. Upgren, \& K.A. Janes, Union College (Schenectady, NY: Davis Press)

Haywood, M. 2008, MNRAS, 388, 1175

Heger, A., Langer, N., \& Woosley, S. E. 2000, ApJ, 528, 368

Helfer, H. L., Wallerstein, G., \& Greenstein, J. L. 1959, ApJ, 129, 700

Hinkel, N. R., \& Kane, S. R. 2013, MNRAS, 432, L36

Hix, W. R., \& Thielemann, F.-K. 1996, ApJ, 460, 869

Hoffman, R. D., Woosley, S. E., Fuller, G. M., \& Meyer, B. S. 1996, ApJ, 460, 478

Holweger, H. 1979, In: The elements and their isotopes in the universe; International Conference on Astrophysics, 22, 117

Huang, C., Zhao, G., Zhang, H. W., \& Chen, Y. Q. 2005, MNRAS, 363, 71

Iben, I. 1991, in Evolution of Stars: The Photospheric Abundance Connection: Proceedings of the 145th Symposium of the International Astronomical Union, IAU (Zlatni Pjasaci, Bulgaria: Kluwer Academic Publishers)

Iben, Jr., I. 1982, ApJ, 260, 821

Iglesias, C. A., \& Rogers, F. J. 1996, ApJ, 464, 943

Ivanova, D. V., \& Shimanskiü, V. V. 2000, Astronomy Reports, 44, 376

Jonsell, K., Edvardsson, B., Gustafsson, B., Magain, P., Nissen, P. E., \& Asplund, M. 2005, A\&A, 440,321

José, J., \& Iliadis, C. 2011, Reports on Progress in Physics, 74, 096901 
Kang, W., \& Lee, S.-G. 2012, MNRAS, 425, 3162

Kang, W., Lee, S.-G., \& Kim, K.-M. 2011, ApJ, 736, 87

Käppeler, F., Gallino, R., Bisterzo, S., \& Aoki, W. 2011, Reviews of Modern Physics, 83, 157

Karakas, A., \& Lattanzio, J. C. 2007, PASA, 24, 103

Karakas, A. I. 2010, MNRAS, 403, 1413

Karakas, A. I., Lugaro, M., Ugalde, C., Wiescher, M., \& Görres, J. 2006, NARs, 50, 500

Kashiv, Y., Davis, A. M., Gallino, R., Cai, Z., Lai, B., Sutton, S. R., \& Clayton, R. N. 2010, ApJ, 713,212

Kobayashi, C., \& Nakasato, N. 2011, ApJ, 729, 16

Kobayashi, C., Umeda, H., Nomoto, K., Tominaga, N., \& Ohkubo, T. 2006, ApJ, 653, 1145

Koch, A., \& Edvardsson, B. 2002, A\&A, 381, 500

Kordopatis, G., et al. 2011, A\&A, 535, A107

Korotin, S., Mishenina, T., Gorbaneva, T., \& Soubiran, C. 2011, MNRAS, 415, 2093

Krumholz, M. R., Klein, R. I., \& McKee, C. F. 2007, ApJ, 656, 959

Kupka, F., Piskunov, N., Ryabchikova, T. A., Stempels, H. C., \& Weiss, W. W. 1999, A\&AS, 138, 119

Kurucz, R. L. 1993, SYNTHE spectrum synthesis programs and line data (Cambridge, MA: Smithsonian Astrophysical Observatory, —c1993)

Kurucz, R. L. 2005, MemSAIS, 8, 14

Lai, D. K., Johnson, J. A., Bolte, M., \& Lucatello, S. 2007, ApJ, 667, 1185

Laird, J. 1985, ApJ, 289, 556

Lambert, D. L. 1989, in American Institute of Physics Conference Series, Vol. 183, Cosmic Abundances of Matter, ed. C. J. Waddington, 168-199

Lambert, D. L., \& Warner, B. 1968, MNRAS, 138, 181

Langanke, K., Feldmeier, H., Martínez-Pinedo, G., \& Neff, T. 2007, Progress in Particle and Nuclear Physics, 59, 66

Lebzelter, T., et al. 2012, A\&A, 547, A108

Limongi, M., \& Chieffi, A. 2003, ApJ, 592, 404 
Limongi, M., \& Chieffi, A. 2006, ApJ, 647, 483

Lodders, K., Plame, H., \& Gail, H.-P. 2009, Landolt-Börnstein - Group VI Astronomy and Astrophysics Numerical Data and Functional Relationships in Science and Technology Volume 4B: Solar System. Edited by J.E. Trümper, 4B, 44

Luck, R. E., \& Heiter, U. 2005, AJ, 129, 1063

—. 2006, AJ, 131, 3069

Magain, P. 1989, A\&A, 209, 211

Malinie, G., Hartmann, D. H., Clayton, D. D., \& Mathews, G. J. 1993, ApJ, 413, 633

Marcolini, A., Gibson, B. K., Karakas, A. I., \& Sánchez-Blázquez, P. 2009, MNRAS, 395, 719

Mashonkina, L., \& Gehren, T. 2000, A\&A, 364, 249

Mashonkina, L., \& Zhao, G. 2006, A\&A, 456, 313

Mashonkina, L. I., Vinogradova, A. B., Ptitsyn, D. A., Khokhlova, V. S., \& Chernetsova, T. A. 2007, Astronomy Reports, 51, 903

Matteucci, F., \& Greggio, L. 1986, A\&A, 154, 279

Matteucci, F., \& Recchi, S. 2001, ApJ, 558, 351

McWilliam, A. 1997, ARA\&A, 35, 503

McWilliam, A., Rich, R. M., \& Smecker-Hane, T. A. 2003, ApJ, 592, L21

Meynet, G., \& Maeder, A. 2002a, A\&A, 390, 561

Mishenina, T. V., Gorbaneva, T. I., Basak, N. Y., Soubiran, C., \& Kovtyukh, V. V. 2011a, Astronomy Reports, 55, 689

—. 2011b, Astronomy Reports, 55, 689

Mishenina, T. V., Kovtyukh, V. V., Korotin, S. A., \& Soubiran, C. 2003, Astronomy Reports, 47, 422

Mishenina, T. V., Soubiran, C., Bienaymé, O., Korotin, S. A., Belik, S. I., Usenko, I. A., \& Kovtyukh, V. V. 2008, A\&A, 489, 923

Mishenina, T. V., Soubiran, C., Kovtyukh, V. V., \& Korotin, S. A. 2004, A\&A, 418, 551

Neuforge-Verheecke, C., \& Magain, P. 1997, A\&A, 328, 261

Neves, V., Santos, N. C., Sousa, S. G., Correia, A. C. M., \& Israelian, G. 2009, A\&A, 497, 563 
Nissen, P. E., \& Schuster, W. J. 1997, A\&A, 326, 751

-. 2010, A\&A, 511, L10

Nissen, P. E., \& Schuster, W. J. 2011, A\&A, 530, A15

Pace, N. 2001, Proceedings of the National Academy of the Sciences, 98, 805

Pagano, M. D. 2014, PhD thesis, Arizona State University

Pagel, B. E. J. 1997, Nucleosynthesis and Chemical Evolution of Galaxies (Cambridge University Press)

Peterson, R. C. 1981, ApJ, 244, 989

Petigura, E. A., \& Marcy, G. W. 2011, ApJ, 735, 41

Petigura, E. A., \& Marcy, G. W. 2011, ApJ, 735, 41

Porto de Mello, G. F., Lyra, W., \& Keller, G. R. 2008, A\&A, 488, 653

Prantzos, N. 2008, EAS Publications Series, 32, 311

Prantzos, N. 2011, ArXiv e-prints

Pritzl, B. J., Venn, K. A., \& Irwin, M. 2005, AJ, 130, 2140

Prochaska, J. X., \& McWilliam, A. 2000, ApJ, 537, L57

Qian, Y.-Z., \& Wasserburg, G. J. 2008, ApJ, 687, 272

Ramírez, I., Fish, J. R., Lambert, D. L., \& Allende Prieto, C. 2012, ApJ, 756, 46

Ramírez, I., Meléndez, J., \& Asplund, M. 2009, A\&A, 508, L17

Ramírez, I., Prieto, C. A., \& Lambert, D. L. 2007, A\&A, 465, 271

Rauscher, T., Heger, A., Hoffman, R. D., \& Woosley, S. E. 2002, ApJ, 576, 323

Recio-Blanco, A., de Laverny, P., Worley, C., Santos, N. C., Melo, C., \& Israelian, G. 2012, A\&A, 538, A117

Reddy, B., Lambert, D., \& Prieto, C. A. 2006, MNRAS, 367, 1

Reddy, B., Tomkin, J., Lambert, D., \& Prieto, C. A. 2003, MNRAS, 340, 304

Reylé, C., \& Robin, A. C. 2001, A\&A, 373, 886

Robles, J. A., Lineweaver, C. H., Grether, D., Flynn, C., Egan, C. A., Pracy, M. B., Holmberg, J., \& Gardner, E. 2008a, ApJ, 684, 691 
-. 2008b, ApJ, 689, 1457

Roederer, I. U. 2013, AJ, 145, 26

Roederer, I. U., Cowan, J. J., Karakas, A. I., Kratz, K., Lugaro, M., Simmerer, J., Farouqi, K., \& Sneden, C. 2010a, ApJ, 724, 975

Roederer, I. U., Lawler, J. E., Sneden, C., Cowan, J. J., Sobeck, J. S., \& Pilachowski, C. A. 2008, ApJ, 675, 723

Roederer, I. U., Sneden, C., Thompson, I. B., Preston, G. W., \& Shectman, S. A. 2010b, ApJ, 711, 573

Romano, D., Karakas, A. I., Tosi, M., \& Matteucci, F. 2010, A\&A, 522, A32

Russell, H. N. 1929, ApJ, 70, 11

Sadakane, K., Ohkubo, M., Takeda, Y., Sato, B., Kambe, E., \& Aoki, W. 2002, PASJ, 54, 911

Sadakane, K., Ohkubo, M., Takeda, Y., Sato, B., Kambe, E., \& Aoki, W. 2002, PASJ, 54, 911

Sandage, A., \& Fouts, G. 1987, AJ, 93, 74

Santos, N. C., Israelian, G., \& Mayor, M. 2004, A\&A, 415, 1153

Santos, N. C., Israelian, G., Mayor, M., Bento, J. P., Almeida, P. C., Sousa, S. G., \& Ecuvillon, A. 2005, A\&A, 437, 1127

Schuler, S. C., Flateau, D., Cunha, K., King, J. R., Ghezzi, L., \& Smith, V. V. 2011, ApJ, 732, 55

Sellwood, J. A., \& Binney, J. J. 2002, MNRAS, 336, 785

Shi, J. R., Gehren, T., \& Zhao, G. 2004, A\&A, 423, 683

Siess, L., Livio, M., \& Lattanzio, J. 2002, ApJ, 570, 329

Simmerer, J., Sneden, C., Cowan, J. J., Collier, J., Woolf, V. M., \& Lawler, J. E. 2004, ApJ, 617, 1091

Sneden, C., Cowan, J. J., \& Gallino, R. 2008, ARA\&A, 46, 241

Sneden, C. A. 1973, PhD thesis, University of Texas, Austin, Texas

Soubiran, C., \& Girard, P. 2005, A\&A, 438, 139

Sousa, S. G., et al. 2008, A\&A, 487, 373

Spite, M. 1967, Annales d'Astrophysique, 30, 211

Spite, M., \& Spite, F. 1978, A\&A, 67, 23 
Suess, H. E., \& Urey, H. C. 1956, Reviews of Modern Physics, 28, 53

Takeda, Y. 2007, PASJ, 59, 335

Takeda, Y., Hashimoto, O., Taguchi, H., Yoshioka, K., Takada-Hidai, M., Saito, Y., \& Honda, S. 2005, PASJ, 57, 751

Takeda, Y., \& Honda, S. 2005, PASJ, 57, 65

Takeda, Y., Taguchi, H., Yoshioka, K., Hashimoto, O., Aikawa, T., \& Kawanomoto, S. 2007, PASJ, 59,1127

Talbot, R., \& Arnett, D. W. 1974, ApJ, 190, 605

Thevenin, F. 1998, Chemical Abundances in Late-Type Stars (VizieR On-line Data Catalog)

Thévenin, F., \& Idiart, T. P. 1999, ApJ, 521, 753

Thielemann, F. K., \& Arnett, W. D. 1985, ApJ, 295, 604

Thielemann, F.-K., Nomoto, K., \& Hashimoto, M.-A. 1996, ApJ, 460, 408

Thielemann, F.-K., et al. 2002, Ap\&SS, 281, 25

—. 2007, Progress in Particle and Nuclear Physics, 59, 74

Timmes, F. X., Woosley, S. E., \& Weaver, T. A. 1995, ApJS, 98, 617

Tinsley, B. M. 1980, Fundamentals of Cosmic Physics, 5, 287

Tolstoy, E., Venn, K. A., Shetrone, M., Primas, F., Hill, V., Kaufer, A., \& Szeifert, T. 2003, AJ, 125,707

Torres, G., Fischer, D. A., Sozzetti, A., Buchhave, L. A., Winn, J. N., Holman, M. J., \& Carter, J. A. 2012, ApJ, 757, 161

Travaglio, C., Galli, D., Gallino, R., Busso, M., Ferrini, F., \& Straniero, O. 1999, ApJ, 521, 691

Trevisan, M., Barbuy, B., Eriksson, K., Gustafsson, B., Grenon, M., \& Pompéia, L. 2011, A\&A, 535,42

Truran, J. W., \& Cameron, A. G. W. 1971, Ap\&SS, 14, 179

Tsymbal, V. 1996, in Astronomical Society of the Pacific Conference Series, Vol. 108, M.A.S.S., Model Atmospheres and Spectrum Synthesis, ed. S. J. Adelman, F. Kupka, \& W. W. Weiss, 198

Tuli, J. K. 2005, in International Conference on Nuclear Data for Science and Technology AIP Conference Proceedings (Santa Fe, NM: AIP) 
Twarog, B. A. 1980, ApJ, 242, 242

Umeda, H., \& Nomoto, K. 2002, ApJ, 565, 385

Valenti, J. A., \& Fischer, D. A. 2005, ApJS, 159, 141

van den Hoek, L. B., \& de Jong, T. 1997, A\&A, 318, 231

Vangioni, E., Silk, J., Olive, K. A., \& Fields, B. D. 2011, MNRAS, 413, 2987

Venn, K. A., Irwin, M., Shetrone, M. D., Tout, C. A., Hill, V., \& Tolstoy, E. 2004, AJ, 128, 1177

Ventura, P., D’antona, F., \& Mazzitelli, I. 2002, A\&A, 393, 215

Wallerstein, G., et al. 1997, Reviews of Modern Physics, 69, 995

Wang, L., Liu, Y., Zhao, G., \& Sato, B. 2011, PASJ, 63, 1035

Wheeler, J. C., Sneden, C., \& Truran, J. W. 1989, ARA\&A, 27, 279

Wielen, R., Fuchs, B., \& Dettbarn, C. 1996, A\&A, 314, 438

Woosley, S. E., Arnett, W. D., \& Clayton, D. D. 1973, ApJS, 26, 231

Woosley, S. E., \& Weaver, T. A. 1995, ApJS, 101, 181

Zhang, H. W., Gehren, T., \& Zhao, G. 2008, eprint arXiv, 0802, 2609

Zhang, H. W., \& Zhao, G. 2006, A\&A, 449, 127

Zhao, G., Chen, Y.-Q., \& Qin, H.-M. 2002, Chinese J. Astron. Astrophys., 2, 377 